\title{
DFT Study of Acceptorless Alcohol Dehydrogenation Mediated by Ruthenium Pincer Complexes: Ligand Tautomerization Governing Metal Ligand Cooperation
}

\author{
Cheng Hou, Zhihan Zhang, Cunyuan Zhao* and Zhuofeng Ke* \\ MOE Key Laboratory of Bioinorganic and Synthetic Chemistry, School of Chemistry and \\ Chemical Engineering, School of Materials Science and Engineering, Sun Yat-sen University, \\ Guangzhou 510275, P. R. China
}

*Author to whom correspondence should be addressed at: kezhf3@mail.sysu.edu.cn

\section{Table of Contents}

1. The comparison of the energy barrier using different functionals ............................2

2. The absolute energies of all optimized structures. ...................................................3

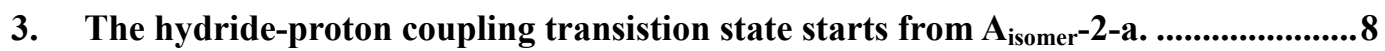

4. The $\mathrm{Ru}^{\mathrm{II}} / \mathrm{Ru}^{0}$ mechanism for Ru-NNN system proposed by Suresh et al....................9

5. The inner-sphere mechanism for NNN-Ru system via ligand rotation ......................10

6. The possibility dissociation of $\mathrm{CO}$ ligand...........................................................11

7. The Cartesian coordinates (xyz) for all optimized structures are presented .............12

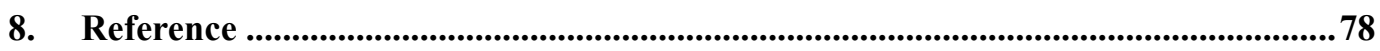




\section{The comparison of the energy barrier using different functionals}

\begin{tabular}{|c|c|c|}
\hline Functionals & B-TS1 $1_{0}-P S$ & B-TS3 \\
\hline M06L/BSII//M06L/BSI & 11.6 & 24.0 \\
\hline B3LYP-D3/BSII// B3LYP-D3/BSI & 7.0 & 23.4 \\
\hline M06/BSII//M06/BSI & 10.9 & 21.6 \\
\hline$\omega \mathrm{B} 97 \mathrm{XD} / \mathrm{BSII} / / \omega \mathrm{B} 97 \mathrm{XD} / \mathrm{BSI}$ & 6.8 & 24.3 \\
\hline BMK /BSII// BMK /BSI & 6.0 & 31.3 \\
\hline
\end{tabular}

Table S1. The comparison of energy barrier using different functionals

- BSII: 6-31G(d, p)/Ru:SDD; BS2: 6-311++G(d,p)/Ru: SDD;

In order to select appropriate functional for our calculation. We compare a series of functionals which are widely used in the computational studies relevant to catalytic hydrogenation and dehydrogenation reaction. The results are shown in Table S1. We choose two transition states (B-TS1 $1_{\mathbf{O}}-\mathbf{P S}$ and $\mathbf{B}-\mathbf{T S 3}_{\mathbf{I}}$ ) in Ru-NNN system as the example for comparison. For most functionals which involve dispersion effect, the results are similar. Based on these results, we choose M06-L as the functional considering the computational expense and accuracy.

Table S2. The comparison of energy barrier using different functionals

\begin{tabular}{ccc}
\hline ECP & $\Delta G^{\ddagger}(\mathrm{kcal} / \mathbf{m o l})$ & $\Delta G^{\ddagger}(\mathrm{kcal} / \mathbf{m o l})$ \\
& A-4I $\rightarrow$ A-TS2I & A-1 $\rightarrow$ A-TS2 $\mathbf{O}_{\mathbf{0}}$-PS \\
\hline SDD & 19.2 & 25.7 \\
AUG-cc-pVTZ-PP & 20.4 & 26.4 \\
\hline
\end{tabular}

We also test the ECP for the Ru atom. The result of the AUG-cc-pVTZ-PP ECP is basically consistent with the SDD data. The predication of mechanistic preference also remain unchanged. Therefore, we believe the SDD data is convincible. In order to reduce the computational expense, we choose the data based on BSII for the single point energy calculation. 


\section{The absolute energies of all optimized structures.}

Table S3. The absolute energies of all optimized structures

\begin{tabular}{|c|c|c|c|c|c|c|c|c|c|c|}
\hline Species & $\begin{array}{l}\text { Zero }_{\mathbf{c}} \\
\text { (BSI) }\end{array}$ & $\begin{array}{c}\mathrm{H}_{\mathrm{c}} \\
\text { (BSI) }\end{array}$ & $\begin{array}{c}\mathbf{G}_{\mathrm{c}} \\
(\mathrm{BSI})\end{array}$ & $\begin{array}{c}\mathbf{E}_{0} \\
\text { (BSI) }\end{array}$ & $\begin{array}{c}\mathrm{H} \\
\text { (BSI) }\end{array}$ & $\begin{array}{c}\text { G } \\
\text { (BSI) }\end{array}$ & $\begin{array}{c}\text { SP+SOL } \\
\text { (BSII) }\end{array}$ & $\begin{array}{c}\text { SP } \\
\text { (BSII) }\end{array}$ & $\begin{array}{c}\text { Sol G } \\
\text { kcal/mol }\end{array}$ & $\begin{array}{c}\text { Final } \\
\text { Free energy }\end{array}$ \\
\hline $\mathrm{H}_{2}$ & 0.009991 & 0.013296 & -0.001498 & -1.160086 & -1.156781 & -1.171575 & -1.17912 & -1.179571 & 0.28 & -1.17376552 \\
\hline PEA & 0.161653 & 0.170998 & 0.128254 & -385.886207 & -385.876862 & -385.919606 & -386.150086 & -386.138568 & -7.23 & -386.0149795 \\
\hline ACP & 0.138211 & 0.14694 & 0.105593 & -384.762122 & -384.753393 & -384.794741 & -384.955544 & -384.943158 & -7.77 & -384.8430985 \\
\hline \multicolumn{11}{|l|}{ Isomerization } \\
\hline A-1 & 0.609293 & 0.647877 & 0.539983 & -2176.696128 & -2176.657544 & -2176.765439 & -2177.729839 & -2177.689064 & -25.59 & -2177.183004 \\
\hline Tsiso-1 & 0.609188 & 0.646852 & 0.542745 & -2176.667776 & -2176.630112 & -2176.734219 & -2177.700091 & -2177.66001 & -25.15 & -2177.150494 \\
\hline Aisomer-1-a & 0.609655 & 0.647942 & 0.542053 & -2176.705305 & -2176.667018 & -2176.772907 & -2177.741813 & -2177.69914 & -26.78 & -2177.192908 \\
\hline Aisomer-2-a & 0.77395 & 0.821434 & 0.696226 & -2562.588893 & -2562.541409 & -2562.666617 & -2563.87756 & -2563.833594 & -27.59 & -2563.174482 \\
\hline Aisomer-2-a-TS & 0.76918 & 0.815951 & 0.692573 & -2562.565744 & -2562.518974 & -2562.642352 & -2563.847205 & -2563.804875 & -26.56 & -2563.14778 \\
\hline \multicolumn{11}{|l|}{ Outer-sphere } \\
\hline A-1 & 0.609293 & 0.647877 & 0.539983 & -2176.696128 & -2176.657544 & -2176.765439 & -2177.729839 & -2177.689064 & -25.59 & -2177.183004 \\
\hline A-TS1o-PS & 0.933397 & 0.989685 & 0.844267 & -2948.484471 & -2948.428183 & -2948.573602 & -2950.023491 & -2949.974849 & -30.52 & -2949.172372 \\
\hline A-TS1o & 0.766515 & 0.812534 & 0.691538 & -2562.550811 & -2562.504792 & -2562.625788 & -2563.830273 & -2563.787809 & -26.65 & -2563.131883 \\
\hline A-2o & 0.629132 & 0.668294 & 0.560107 & -2177.843891 & -2177.80473 & -2177.912916 & -2178.899574 & -2178.858479 & -25.79 & -2178.332615 \\
\hline A-TS2o & 0.62307 & 0.661532 & 0.555923 & -2177.806681 & -2177.768218 & -2177.873828 & -2178.853011 & -2178.814583 & -24.11 & -2178.290236 \\
\hline A-TS2o-PS & 0.951096 & 1.008426 & 0.860497 & -2949.653661 & -2949.596331 & -2949.74426 & -2951.211188 & -2951.16516 & -28.88 & -2950.343839 \\
\hline \multicolumn{11}{|l|}{$\beta$-Elimination } \\
\hline A-1 & 0.609293 & 0.647877 & 0.539983 & -2176.696128 & -2176.657544 & -2176.765439 & -2177.729839 & -2177.689064 & -25.59 & -2177.183004 \\
\hline
\end{tabular}




\begin{tabular}{|c|c|c|c|c|c|c|c|c|c|c|}
\hline A-2I & 0.774361 & 0.822188 & 0.69568 & -2562.61782 & -2562.569994 & -2562.696501 & -2563.902254 & -2563.862835 & -24.74 & -2563.199722 \\
\hline A-TS1I-PS & 0.934354 & 0.990469 & 0.847132 & -2948.521514 & -2948.465399 & -2948.608736 & -2950.057095 & -2950.012367 & -28.07 & -2949.203111 \\
\hline A-TS1I & 0.769589 & 0.816828 & 0.691538 & -2562.59965 & -2562.552411 & -2562.677701 & -2563.878417 & -2563.839082 & -24.68 & -2563.180027 \\
\hline A-3I & 0.772578 & 0.819948 & 0.694629 & -2562.612756 & -2562.565386 & -2562.690705 & -2563.896437 & -2563.856077 & -25.33 & -2563.194956 \\
\hline A-3I & 0.752633 & 0.798963 & 0.676457 & -2561.457819 & -2561.411489 & -2561.533994 & -2562.719765 & -2562.678916 & -25.63 & -2562.036456 \\
\hline A-TS2I & 0.74945 & 0.795879 & 0.673223 & -2561.42721 & -2561.38078 & -2561.503436 & -2562.685962 & -2562.643481 & -26.66 & -2562.005887 \\
\hline A-5I & 0.750785 & 0.797977 & 0.671854 & -2561.448041 & -2561.40085 & -2561.526972 & -2562.711935 & -2562.667361 & -27.97 & -2562.033229 \\
\hline \multicolumn{11}{|l|}{ ligand rotation } \\
\hline A-1 & 0.609293 & 0.647877 & 0.539983 & -2176.696128 & -2176.657544 & -2176.765439 & -2177.729839 & -2177.689064 & -25.59 & -2177.183004 \\
\hline A-rot-TS1I & 0.769299 & 0.814493 & 0.695664 & -2562.57235 & -2562.527156 & -2562.645985 & -2563.850654 & -2563.810052 & -25.48 & -2563.148138 \\
\hline A-rot-TS1I-PS & 0.935505 & 0.991799 & 0.84826 & -2948.498844 & -2948.44255 & -2948.586089 & -2950.036982 & -2949.992742 & -27.76 & -2949.18187 \\
\hline \multicolumn{11}{|l|}{ Ru-PNN } \\
\hline \multicolumn{11}{|l|}{ Isomerization } \\
\hline B-1 & 0.519867 & 0.550345 & 0.462534 & -1403.704998 & -1403.674521 & -1403.762331 & -1404.47971 & -1404.457447 & -13.97 & -1404.010324 \\
\hline B-TSiso-1 & 0.518533 & 0.548715 & 0.461715 & -1403.632894 & -1403.602711 & -1403.689711 & -1404.403767 & -1404.38492 & -11.83 & -1403.9352 \\
\hline Bisomer-1-a & 0.520693 & 0.550826 & 0.464438 & -1403.660918 & -1403.630785 & -1403.717173 & -1404.438353 & -1404.413921 & -15.33 & -1403.967063 \\
\hline Bisomer-2-a & 0.685492 & 0.724526 & 0.620011 & -1789.593814 & -1789.554781 & -1789.659295 & -1790.622433 & -1790.5971 & -15.9 & -1789.99557 \\
\hline B-TSiso-2 & 0.518202 & 0.548194 & 0.46229 & -1403.655267 & -1403.625276 & -1403.71118 & -1404.428557 & -1404.405638 & -14.38 & -1403.959415 \\
\hline Bisomer-1-b & 0.519339 & 0.549643 & 0.463095 & -1403.697025 & -1403.66672 & -1403.753269 & -1404.469973 & -1404.448399 & -13.54 & -1404.000026 \\
\hline Bisomer-2-b & 0.685807 & 0.725181 & 0.619089 & -1789.592991 & -1789.553617 & -1789.65971 & -1790.623399 & -1790.596791 & -16.7 & -1789.997458 \\
\hline \multicolumn{11}{|l|}{ Outer-sphere } \\
\hline B-1 & 0.519867 & 0.550345 & 0.462534 & -1403.704998 & -1403.674521 & -1403.762331 & -1404.47971 & -1404.457447 & -13.97 & -1404.010324 \\
\hline B-TS1o & 0.680177 & 0.718512 & 0.615394 & -1789.600856 & -1789.562521 & -1789.665638 & -1790.626566 & -1790.598196 & -17.8 & -1790.00432 \\
\hline B-TS1o-PS & 0.843508 & 0.891156 & 0.767099 & -2175.512926 & -2175.465278 & -2175.589335 & -2176.795682 & -2176.760044 & -22.36 & -2176.021731 \\
\hline
\end{tabular}




\begin{tabular}{|c|c|c|c|c|c|c|c|c|c|c|}
\hline B-2o & 0.540316 & 0.570253 & 0.485385 & -1404.890825 & -1404.860887 & -1404.945756 & -1405.684349 & -1405.662044 & -14 & -1405.192112 \\
\hline B-TS2o & 0.534888 & 0.564805 & 0.479181 & -1404.83578 & -1404.805864 & -1404.891488 & -1405.622617 & -1405.603064 & -12.27 & -1405.136584 \\
\hline B-TS2o-PS & 0.698339 & 0.737347 & 0.632343 & -1790.766674 & -1790.727667 & -1790.832671 & -1791.810101 & -1791.784774 & -15.89 & -1791.170906 \\
\hline \multicolumn{11}{|l|}{ Inner-sphere } \\
\hline B-1 & 0.519867 & 0.550345 & 0.462534 & -1403.704998 & -1403.674521 & -1403.762331 & -1404.47971 & -1404.457447 & -13.97 & -1404.010324 \\
\hline B-2I & 0.686459 & 0.725711 & 0.620306 & -1789.622092 & -1789.58284 & -1789.688245 & -1790.651476 & -1790.626449 & -15.7 & -1790.024318 \\
\hline B-TS1I & 0.682555 & 0.720474 & 0.618854 & -1789.60268 & -1789.564761 & -1789.666381 & -1790.624395 & -1790.602222 & -13.91 & -1789.998689 \\
\hline B-TS1I-PS & 0.847192 & 0.894245 & 0.773558 & -2175.532147 & -2175.485094 & -2175.60578 & -2176.810993 & -2176.783337 & -17.35 & -2176.030583 \\
\hline B-3I & 0.686376 & 0.724745 & 0.621536 & -1789.635651 & -1789.597282 & -1789.700491 & -1790.664809 & -1790.639225 & -16.05 & -1790.036421 \\
\hline B-TS2I & 0.683951 & 0.722819 & 0.615999 & -1789.608512 & -1789.569644 & -1789.676465 & -1790.636992 & -1790.610869 & -16.39 & -1790.014141 \\
\hline B-4I & 0.685513 & 0.724768 & 0.617153 & -1789.613212 & -1789.573957 & -1789.681572 & -1790.642822 & -1790.616853 & -16.3 & -1790.018817 \\
\hline B-TS3I & 0.679771 & 0.719007 & 0.611935 & -1789.594095 & -1789.55486 & -1789.661931 & -1790.616965 & -1790.590494 & -16.61 & -1789.998178 \\
\hline B-5I & 0.68143 & 0.721056 & 0.612384 & -1789.60513 & -1789.565504 & -1789.674175 & -1790.628473 & -1790.602899 & -16.05 & -1790.009237 \\
\hline B-TS4I & 0.538242 & 0.568773 & 0.480798 & -1404.850172 & -1404.819641 & -1404.907616 & -1405.644056 & -1405.621035 & -14.45 & -1405.156406 \\
\hline $\mathrm{H} 2$ & 0.009991 & 0.013296 & -0.001498 & -1.160086 & -1.156781 & -1.171575 & -1.17912 & -1.179571 & 0.28 & -1.17376552 \\
\hline $\mathrm{PE}$ & 0.161653 & 0.170998 & 0.128254 & -385.886207 & -385.876862 & -385.919606 & -386.150086 & -386.138568 & -7.23 & -386.0149795 \\
\hline ACP & 0.138211 & 0.14694 & 0.105593 & -384.762122 & -384.753393 & -384.794741 & -384.955544 & -384.943158 & -7.77 & -384.8430985 \\
\hline \multicolumn{11}{|l|}{ Tautomerization } \\
\hline Ligand A-1 & 0.337558 & 0.358526 & 0.287446 & -1045.41 & -1045.38616 & -1045.45724 & -1046.03 & -1046 & -19.26 & -1045.73594 \\
\hline Ligand A-2 & 0.334425 & 0.355401 & 0.286222 & -1045.37 & -1045.3487 & -1045.41788 & -1046 & -1045.96 & -23.09 & -1045.70553 \\
\hline Ligand B-1 & 0.517346 & 0.544353 & 0.46269 & -1195 & -1194.97221 & -1195.053876 & -1195.77 & -1195.75 & -11.37 & -1195.30231 \\
\hline Ligand B-2 & 0.516738 & 0.543748 & 0.459412 & -1195.02 & -1194.98816 & -1195.072495 & -1195.79 & -1195.77 & -11.39 & -1195.31938 \\
\hline L-1-a & 0.623465 & 0.656899 & 0.562005 & -1638.66 & -1638.62962 & -1638.724517 & -1639.6 & -1639.58 & -12.35 & -1639.02704 \\
\hline L-1-b & 0.624146 & 0.657079 & 0.564028 & -1638.68 & -1638.64942 & -1638.74247 & -1639.61 & -1639.59 & -12 & -1639.04287 \\
\hline
\end{tabular}




\begin{tabular}{|c|c|c|c|c|c|c|c|c|c|c|}
\hline L-2-a & 0.611325 & 0.644748 & 0.548415 & -1654.74 & -1654.70273 & -1654.799061 & -1655.67 & -1655.64 & -13.7 & -1655.11024 \\
\hline L-2-b & 0.611851 & 0.644823 & 0.551616 & -1654.75 & -1654.71682 & -1654.810027 & -1655.68 & -1655.66 & -13.17 & -1655.1198 \\
\hline L-3-a & 0.42634 & 0.45024 & 0.373914 & -1190.3 & -1190.27147 & -1190.347795 & -1190.98 & -1190.96 & -12.29 & -1190.5962 \\
\hline L-3-b & 0.426222 & 0.45002 & 0.373537 & -1190.31 & -1190.28531 & -1190.361788 & -1190.99 & -1190.97 & -13.33 & -1190.61101 \\
\hline L-4-a & 0.415072 & 0.43882 & 0.362832 & -1206.37 & -1206.34394 & -1206.419923 & -1207.05 & -1207.02 & -13.69 & -1206.67695 \\
\hline L-4-b & 0.414662 & 0.438336 & 0.363412 & -1206.38 & -1206.35328 & -1206.428209 & -1207.06 & -1207.03 & -14.27 & -1206.68614 \\
\hline L-5-a & 0.313458 & 0.331942 & 0.267412 & -859.74 & -859.721461 & -859.785991 & -860.284 & -860.262 & -13.81 & -860.010034 \\
\hline L-5-b & 0.312183 & 0.33143 & 0.262186 & -859.711 & -859.691944 & -859.761188 & -860.257 & -860.233 & -14.93 & -859.987966 \\
\hline L-6-a & 0.28988 & 0.308175 & 0.243349 & -891.854 & -891.835875 & -891.900701 & -892.392 & -892.366 & -16.88 & -892.142272 \\
\hline L-6-b & 0.288336 & 0.307548 & 0.237316 & -891.82 & -891.80108 & -891.871312 & -892.365 & -892.333 & -20.3 & -892.121284 \\
\hline L-7-a & 0.361067 & 0.382237 & 0.311805 & -1013.29 & -1013.26713 & -1013.337564 & -1013.92 & -1013.89 & -16.27 & -1013.59908 \\
\hline L-7-b & 0.359689 & 0.381763 & 0.305653 & -1013.26 & -1013.24092 & -1013.317029 & -1013.89 & -1013.87 & -17.5 & -1013.58166 \\
\hline L-8-a & 0.349217 & 0.370361 & 0.299166 & -1029.35 & -1029.32791 & -1029.3991 & -1029.98 & -1029.95 & -17.98 & -1029.66949 \\
\hline L-8-b & 0.347585 & 0.369667 & 0.293658 & -1029.32 & -1029.29354 & -1029.369549 & -1029.95 & -1029.91 & -20.92 & -1029.64641 \\
\hline L-9-a & 0.505661 & 0.532557 & 0.450437 & -1211.06 & -1211.03189 & -1211.11401 & -1211.83 & -1211.81 & -12.09 & -1211.37019 \\
\hline L-9-b & 0.50492 & 0.531659 & 0.450539 & -1211.07 & -1211.04099 & -1211.122113 & -1211.83 & -1211.81 & -12.19 & -1211.37686 \\
\hline L-10-a & 0.49386 & 0.520669 & 0.438932 & -1227.11 & -1227.0856 & -1227.167334 & -1227.88 & -1227.86 & -14.81 & -1227.43465 \\
\hline L-10-b & 0.493426 & 0.520287 & 0.437529 & -1227.12 & -1227.09483 & -1227.177588 & -1227.89 & -1227.87 & -14.75 & -1227.44418 \\
\hline L-11-a & 0.550346 & 0.579934 & 0.4914 & -1309.44 & -1309.41463 & -1309.503164 & -1310.28 & -1310.26 & -11.91 & -1309.78555 \\
\hline L-11-b & 0.55087 & 0.580237 & 0.494829 & -1309.43 & -1309.40079 & -1309.486196 & -1310.27 & -1310.25 & -11.3 & -1309.76789 \\
\hline L-12-a & 0.521463 & 0.550457 & 0.464975 & -1399.43 & -1399.40048 & -1399.485961 & -1400.28 & -1400.25 & -13.68 & -1399.80348 \\
\hline L-12-b & 0.520947 & 0.550135 & 0.46364 & -1399.43 & -1399.39924 & -1399.485733 & -1400.27 & -1400.25 & -12.77 & -1399.80121 \\
\hline L-13-a & 0.324661 & 0.343688 & 0.277481 & -876.93 & -876.910747 & -876.976955 & -877.495 & -877.472 & -14.89 & -877.210939 \\
\hline L-13-b & 0.324781 & 0.344391 & 0.273359 & -876.968 & -876.947942 & -877.018974 & -877.532 & -877.51 & -14.03 & -877.25185 \\
\hline
\end{tabular}




\begin{tabular}{|c|c|c|c|c|c|c|c|c|c|c|}
\hline L-14-a & 0.312855 & 0.331816 & 0.264856 & -892.995 & -892.976481 & -893.043441 & -893.559 & -893.531 & -17.39 & -893.287386 \\
\hline L-14-b & 0.312715 & 0.332536 & 0.260003 & -893.013 & -892.993228 & -893.06576 & -893.577 & -893.55 & -16.79 & -893.31033 \\
\hline L-15-a & 0.371629 & 0.393394 & 0.320352 & -1030.48 & -1030.45446 & -1030.527501 & -1031.13 & -1031.1 & -16.67 & -1030.79818 \\
\hline L-15-b & 0.372374 & 0.394548 & 0.317899 & -1030.5 & -1030.47866 & -1030.555313 & -1031.15 & -1031.12 & -16.34 & -1030.82569 \\
\hline L-16-a & 0.41618 & 0.439777 & 0.364563 & -1125.08 & -1125.05415 & -1125.129359 & -1125.8 & -1125.77 & -18.1 & -1125.42624 \\
\hline L-16-b & 0.416832 & 0.440839 & 0.36253 & -1125.08 & -1125.05822 & -1125.136529 & -1125.81 & -1125.77 & -19.42 & -1125.43579 \\
\hline $1_{\text {mod }}$ & 0.632974 & 0.671861 & 0.564557 & -2177.838192 & -2177.799305 & -2177.906609 & -2178.896541 & -2178.856726 & -24.98 & -2178.325132 \\
\hline $\mathrm{TS}_{\text {mod }}$ & 0.955889 & 1.012901 & 0.865926 & -2949.641496 & -2949.584484 & -2949.731459 & -2951.205983 & -2951.156422 & -31.1 & -2950.333205 \\
\hline A $-4_{\mathrm{I}}-2$ & 0.611418 & 0.650481 & 0.541933 & -2176.644612 & -2176.605549 & -2176.714098 & -2177.684403 & -2177.640542 & -27.52 & -2177.135618 \\
\hline A-TS3 ${ }^{-}-P S$ & 0.904975 & 0.961025 & 0.817098 & -2947.272325 & -2947.216276 & -2947.360202 & -2948.783245 & -2948.733369 & -31.3 & -2947.98543 \\
\hline $\mathrm{A}^{-T S 3_{I}}$ & 0.747145 & 0.79385 & 0.670869 & -2561.384312 & -2561.337607 & -2561.460588 & -2562.64682 & -2562.601574 & -28.39 & -2561.969099 \\
\hline
\end{tabular}

- The Zero $\mathbf{C}, \mathbf{H}_{\mathbf{C}}$ and $\mathbf{G}_{\mathbf{C}}$ designate the thermal correction to Energy, Enthalpy and Gibbs Free Energy.

- The E, $\mathbf{H}$ and $\mathbf{G}$ designate the Electronic Energy with ZPE correction, Enthalpy and Gibbs Free Energy in gas phase, respectively.

- SP designates the single point energy at BSII level. Sol G designates the solvation free energy calculated by SMD continuum solvent mode. The SP + Sol G designates the sum of single point energy at BSII level and the solvation free energy.

- The Final Free energy results are given with the correction of MHP scheme. 


\section{The hydride-proton coupling transistion state starts from $\mathbf{A}_{\text {isomer-2-a. }}$}

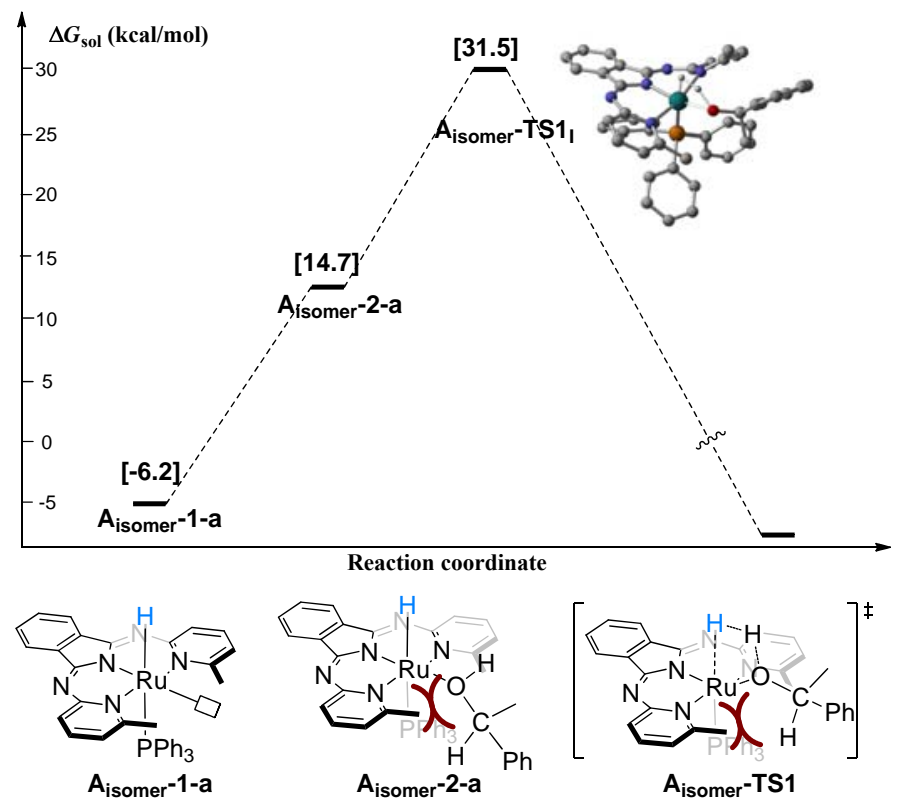

Figure S1. The hydride-proton coupling transistion state $\mathbf{A}_{\text {isomer }}-\mathbf{T S 1}$ I

The transition state of the hydride-proton coupling $\mathbf{A}_{\text {isomer }}-\mathbf{T S 1} 1_{\text {I }}$ is further confirmed with the activation free energy of $37.7 \mathrm{kcal} / \mathrm{mol}$ relative to $\mathbf{A}_{\text {isomer }} \mathbf{- 1 - a}$, which is much higher than the A-TS1 I (11.3 kcal/mol). The proton-shuttle mechanism cannot be located with such a crowded ligand environment. The high barrier is mainly caused by the steric effects between the methyl group on the pincer ligand and the substrate, phenethyl alcohol. On the other hand, the hydride in $\mathbf{A - 1}$ is at the trans-site of a anionic nitrogen atom. Therefore, $\mathbf{A}-\mathbf{1}$ is more active than $\mathbf{A}_{\text {isomer }} \mathbf{- 1 - a}$. 


\section{The $R u^{\mathrm{II}} / \mathrm{Ru}^{0}$ mechanism for $\mathrm{Ru}-\mathrm{NNN}$ system proposed by Suresh et al.}

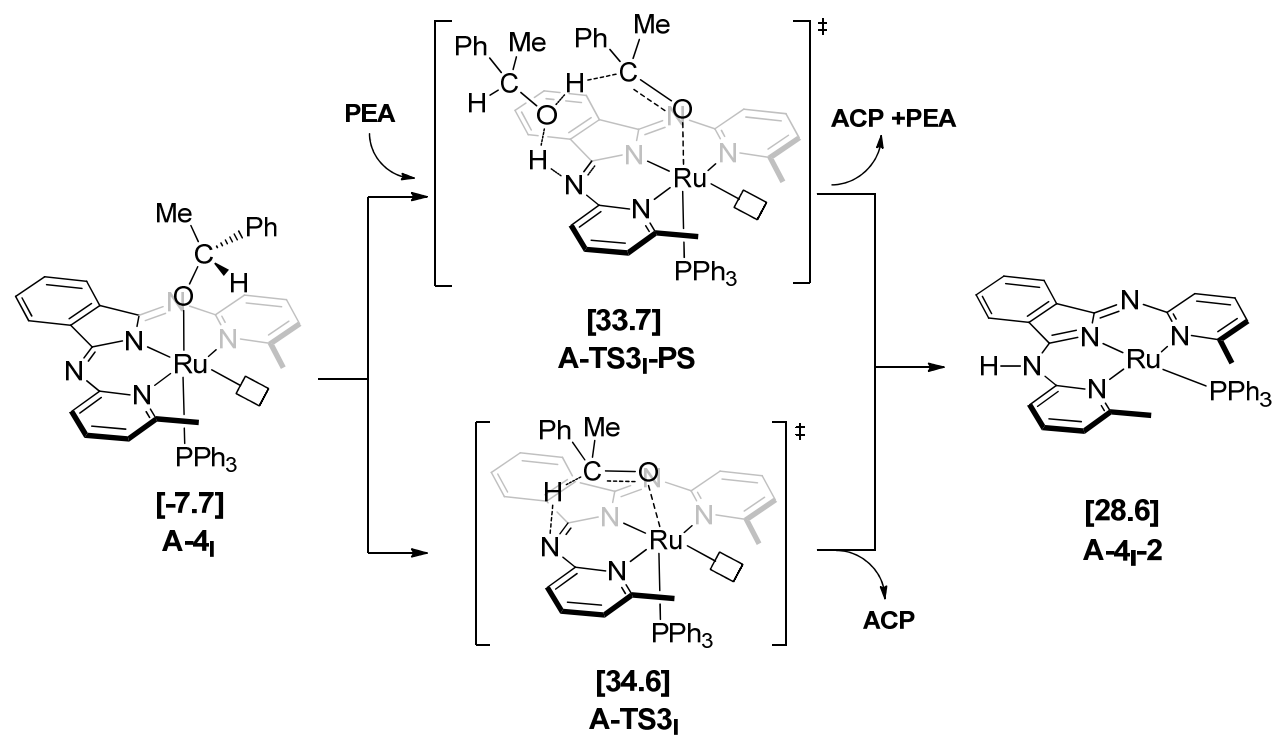

Figure S2. The $\mathrm{Ru}^{\mathrm{II}} / \mathrm{Ru}^{0}$ mechanism for Ru-NNN system proposed by Suresh et al.

The calculated result is depicted in Figure S2. In 2013, Suresh et al proposed a MLC mechanism. ${ }^{1}$ During this mechanism, the $\mathrm{C}-\mathrm{H}$ will transfer the hydrogen atom as proton, and the metal center will be reduced to $\mathrm{Ru}^{0}$ from $\mathrm{Ru}^{\mathrm{II}}$, which leads to a square-planar complex with $d^{8}$ center. As we can see, the $\mathrm{Ru}^{\mathrm{II}} / \mathrm{Ru}^{0}$ mechanism has to overcome much higher barrier in free activation $\left(\Delta \mathrm{G}^{\neq}=34.6 \mathrm{kcal} / \mathrm{mol}\right)$. The proton shuttle type mechanism is also less feasible $\left(\Delta \mathrm{G}^{\neq}=33.7 \mathrm{kcal} / \mathrm{mol}\right)$ Therefore, this mechanism can be ruled out. The high energy barrier is caused by multiple reasons. First of all, the ligand tautomerization is still unfavored for this mechanism, which is reflected in the high free energy of $\mathbf{A}-\mathbf{4}_{\mathrm{I}} \mathbf{-} \mathbf{2}$. Secondly, the $\mathrm{Ru}^{\mathrm{II}}$ is difficult to be reduced to $\mathrm{Ru}^{0}$ under experimental condition. Thirdly, the steric effect makes it difficult to operate this mechanism. 


\section{The inner-sphere mechanism for NNN-Ru system via ligand rotation}

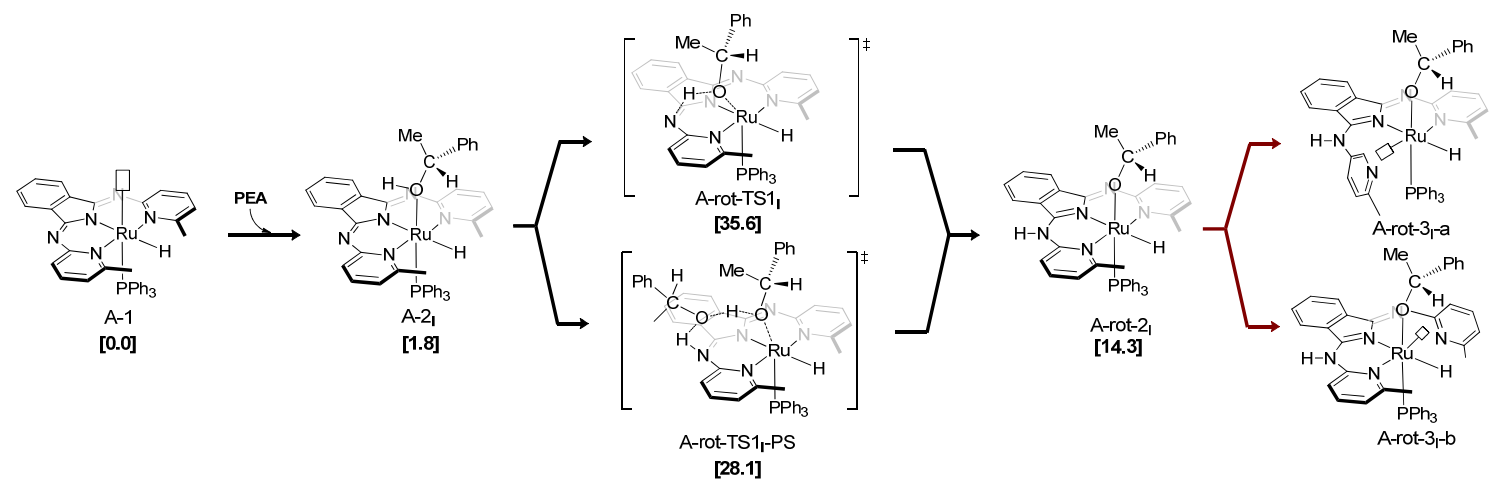

Figure S3. The bifunctional mechanism for NNN-Ru system via ligand rotation

For the ligand rotation mechanism, the $\mathrm{O}-\mathrm{H}$ bond will firstly be cleaved by MLC mechanism. Then, the pyridine ligand will rotate to provide a vacant site for $\beta$-elimination. The cleavage of O-H is found to be very difficult $\left(\Delta \mathrm{G}^{\neq}=28.1 \mathrm{kcal} / \mathrm{mol}\right)$, which is much higher the A-TS2 $\left(\Delta \mathrm{G}^{\ddagger}=19.2 \mathrm{kcal} / \mathrm{mol}\right)$. Thus, the rotation of ligand cannot even occur. This is consistent with the experimental observation. Therefore, this inner-sphere mechanism via ligand rotation is ruled out. 


\section{The possibility dissociation of CO ligand}

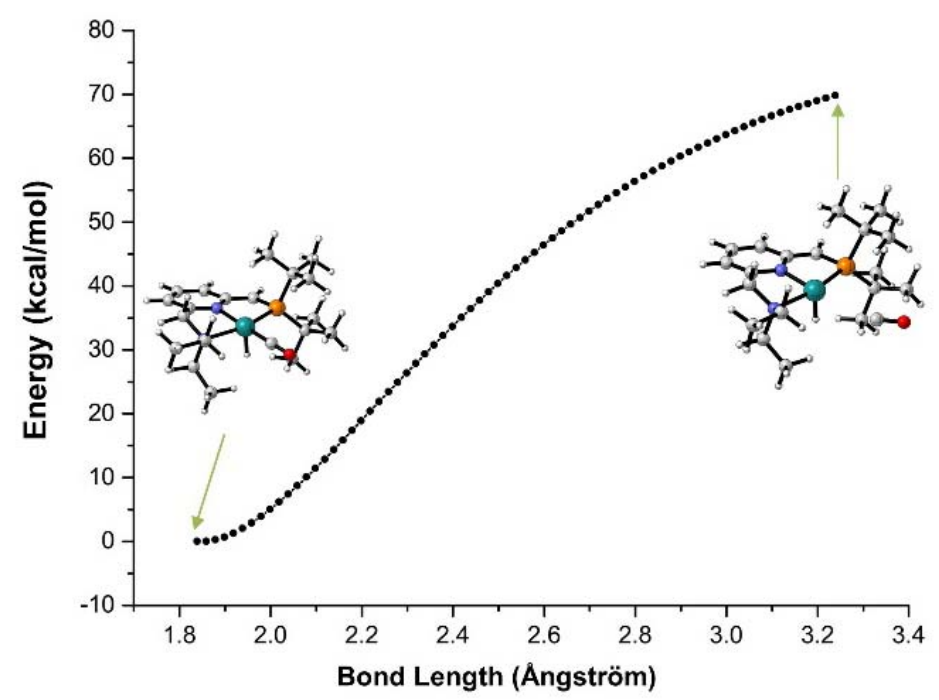

Figure S4. The scan calculation of the Ru-CO bond

For the PNN-Ru system, the dissociation of CO ligand is also considered. We cannot locate the transition state of the dissociation of CO ligand. Therefore, we carried out a scan calculation by enlength the Ru-CO bond. The result indicated that there was not a saddle point when the bond lengh increased. On the other hand, the energy kept increasing ( $~ 70$ $\mathrm{kcal} / \mathrm{mol}$ ) along with the bond length (from $1.84 \AA$ to $3.24 \AA$ ). Therefore, the dissociation of CO seems to be difficult to occur. 


\section{A-TS iso -1}

$\begin{array}{lllll}44 & 0.418219 & -0.011703 & -1.408514\end{array}$

$\begin{array}{llll}1 & 1.479738 & -0.071976 & -2.734634\end{array}$

$\begin{array}{lllll}15 & 0.841758 & -0.025166 & 0.817266\end{array}$

$\begin{array}{lllll}7 & 0.423074 & 2.146606 & -1.589898\end{array}$

$\begin{array}{llll}7 & -1.884642 & 2.400447 & -0.795345\end{array}$

$\begin{array}{llll}7 & -1.535830 & 0.025860 & -0.958345\end{array}$

$\begin{array}{lllll}7 & -1.984566 & -2.339027 & -0.855286\end{array}$

$\begin{array}{llll}7 & 0.379888 & -2.152078 & -1.504248\end{array}$

$\begin{array}{lllll}6 & 1.494570 & 2.854837 & -2.073124\end{array}$

$\begin{array}{lllll}6 & 1.577500 & 4.238705 & -1.971002\end{array}$

$\begin{array}{llll}1 & 2.466579 & 4.731975 & -2.350896\end{array}$

$\begin{array}{lllll}6 & 0.557983 & 4.968114 & -1.364296\end{array}$

$\begin{array}{llll}1 & 0.631138 & 6.045698 & -1.248670\end{array}$

$\begin{array}{llll}6 & -0.552965 & 4.281780 & -0.943993\end{array}$

$1 \quad-1.416523 \quad 4.772840 \quad-0.510486$

$6 \quad-0.645255 \quad 2.880145 \quad-1.106874$

$\begin{array}{llll}6 & -2.282764 & 1.159502 & -0.806015\end{array}$

$\begin{array}{lllll}6 & -3.669090 & 0.763918 & -0.535544\end{array}$

$\begin{array}{lllll}6 & -4.807503 & 1.507229 & -0.264952\end{array}$

$\begin{array}{llll}1 & -4.776211 & 2.592951 & -0.280789\end{array}$

$\begin{array}{llll}6 & -5.979781 & 0.808693 & 0.037513\end{array}$

$\begin{array}{llll}1 & -6.890020 & 1.358715 & 0.261150\end{array}$

$\begin{array}{lllll}6 & -6.000991 & -0.588608 & 0.061036\end{array}$

$\begin{array}{llll}1 & -6.927443 & -1.102517 & 0.303500\end{array}$

$6 \quad-4.851738-1.333198 \quad-0.219012$

$\begin{array}{lllll}1 & -4.853947 & -2.419140 & -0.197317\end{array}$

$6 \quad-3.690539-0.636260-0.512552$

$6 \quad-2.321099-1.083316-0.789572$

$6 \quad-0.767331-2.859254-1.185051$

$6 \quad-0.768833-4.273228-1.187828$

$1 \quad-1.700011-4.751208-0.906706$

$\begin{array}{lllll}6 & 0.351625 & -4.983511 & -1.544160\end{array}$

$1 \quad 0.348934 \quad-6.069775-1.551301$

$6 \quad 1.493496 \quad-4.264514 \quad-1.886858$

$1 \quad 2.415078 \quad-4.772689-2.152615$

$\begin{array}{llll}6 & 1.493461 & -2.874687 & -1.852139\end{array}$

$\begin{array}{llll}6 & 2.647750 & 2.154974 & -2.717227\end{array}$

$\begin{array}{llll}1 & 3.073910 & 1.373628 & -2.081394\end{array}$
$1 \quad 3.424714 \quad 2.883315 \quad-2.967156$

$\begin{array}{llll}1 & 2.335060 & 1.633790 & -3.625402\end{array}$

$\begin{array}{lllll}6 & 2.782954 & -2.180968 & -2.146881\end{array}$

$1 \quad 3.008594 \quad-1.418985-1.394944$

$1 \quad 2.741269-1.651168-3.102156$

$1 \quad 3.595713 \quad-2.913235 \quad-2.172244$

$\begin{array}{llll}6 & 1.421236 & 1.590402 & 1.477589\end{array}$

$\begin{array}{llll}6 & 2.443955 & 2.260026 & 0.791804\end{array}$

$\begin{array}{llll}6 & 0.891328 & 2.188408 & 2.627117\end{array}$

$\begin{array}{llll}6 & 2.911412 & 3.494660 & 1.226210\end{array}$

$\begin{array}{lllll}1 & 2.877971 & 1.793824 & -0.089057\end{array}$

$\begin{array}{llll}6 & 1.350830 & 3.431251 & 3.055140\end{array}$

$\begin{array}{llll}1 & 0.119515 & 1.683555 & 3.198049\end{array}$

$\begin{array}{llll}6 & 2.355394 & 4.091374 & 2.354638\end{array}$

$\begin{array}{llll}1 & 3.701238 & 3.994596 & 0.671336\end{array}$

$\begin{array}{llll}1 & 0.921484 & 3.881014 & 3.946372\end{array}$

$\begin{array}{llll}1 & 2.710286 & 5.061586 & 2.691415\end{array}$

$\begin{array}{llll}6 & 2.212345 & -1.184922 & 1.234059\end{array}$

$\begin{array}{lllll}6 & 3.479186 & -0.717460 & 1.611295\end{array}$

$\begin{array}{lllll}6 & 2.038623 & -2.572307 & 1.092552\end{array}$

$\begin{array}{lllll}6 & 4.535174 & -1.600408 & 1.819948\end{array}$

$\begin{array}{llll}1 & 3.647679 & 0.345262 & 1.752163\end{array}$

$\begin{array}{lllll}6 & 3.091903 & -3.452621 & 1.311286\end{array}$

$\begin{array}{lllll}1 & 1.070857 & -2.975029 & 0.808704\end{array}$

$\begin{array}{lllll}6 & 4.348995 & -2.970418 & 1.666251\end{array}$

$\begin{array}{lllll}1 & 5.507148 & -1.210549 & 2.110261\end{array}$

$\begin{array}{lllll}1 & 2.925697 & -4.520301 & 1.191839\end{array}$

$\begin{array}{lllll}1 & 5.174294 & -3.658536 & 1.827048\end{array}$

$\begin{array}{lllll}6 & -0.503465 & -0.485192 & 1.997409\end{array}$

$\begin{array}{lllll}6 & -0.532789 & -1.674123 & 2.736808\end{array}$

$\begin{array}{llll}6 & -1.624979 & 0.358576 & 2.064541\end{array}$

$\begin{array}{llll}6 & -1.658479 & -2.025012 & 3.479042\end{array}$

$\begin{array}{lllll}1 & 0.329875 & -2.330688 & 2.753683\end{array}$

$\begin{array}{llll}6 & -2.748463 & 0.006862 & 2.801590\end{array}$

$\begin{array}{lllll}1 & -1.614228 & 1.310631 & 1.539634\end{array}$

$\begin{array}{lllll}6 & -2.776470 & -1.198107 & 3.498934\end{array}$

$1 \quad-1.655802-2.952730 \quad 4.045133$

$1 \quad-3.609621 \quad 0.670242 \quad 2.813141$

$1 \quad-3.659528 \quad-1.483391 \quad 4.063724$

\section{$\mathbf{A}_{\text {isomer-1-a }}$}




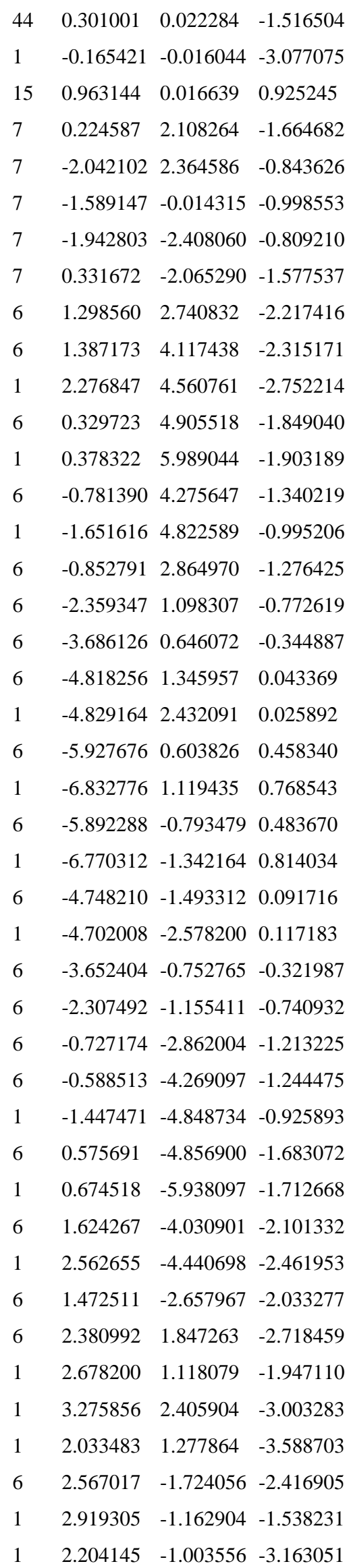




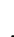$$
\begin{array}{lllll}
7 & 0.163819 & 2.498803 & -0.600634
\end{array}
$$$$
6 \quad-1.700126 \quad-2.250927-1.991019
$$$$
6 \quad-2.032942 \quad-3.587160-1.791525
$$$$
1 \quad-2.979376 \quad-3.951478-2.179021
$$$$
\begin{array}{llll}
6 & -1.134633 & -4.443745 & -1.149609
\end{array}
$$$$
\begin{array}{lllll}
1 & -1.399079 & -5.477898 & -0.946853
\end{array}
$$$$
\begin{array}{lllll}
6 & 0.131112 & -3.972964 & -0.886458
\end{array}
$$$$
\begin{array}{lllll}
1 & 0.935885 & -4.610031 & -0.535544
\end{array}
$$$$
\begin{array}{lllll}
6 & 0.446822 & -2.622732 & -1.150911
\end{array}
$$$$
\begin{array}{lllll}
6 & 2.243076 & -1.149755 & -1.525148
\end{array}
$$$$
\begin{array}{lllll}
6 & 3.625154 & -0.934791 & -1.959997
\end{array}
$$$$
\begin{array}{lllll}
6 & 4.685100 & -1.808072 & -2.141155
\end{array}
$$$$
\begin{array}{lllll}
1 & 4.564297 & -2.871318 & -1.954000
\end{array}
$$$$
\begin{array}{lllll}
6 & 5.906411 & -1.266567 & -2.551696
\end{array}
$$$$
1 \quad 6.761248 \quad-1.920955-2.700272
$$$$
\begin{array}{llll}
6 & 6.048801 & 0.106159 & -2.771560
\end{array}
$$$$
\begin{array}{lllll}
1 & 7.011089 & 0.496586 & -3.091774
\end{array}
$$$$
\begin{array}{lllll}
6 & 4.975167 & 0.982396 & -2.586385
\end{array}
$$$$
\begin{array}{lllll}
1 & 5.075774 & 2.051892 & -2.748179
\end{array}
$$$$
\begin{array}{llll}
6 & 3.766489 & 0.439985 & -2.181568
\end{array}
$$$$
\begin{array}{llll}
6 & 2.479921 & 1.053830 & -1.831586
\end{array}
$$$$
\begin{array}{llll}
6 & 1.211316 & 3.024840 & -1.336294
\end{array}
$$$$
\begin{array}{llll}
6 & 1.296170 & 4.408635 & -1.589144
\end{array}
$$$$
\begin{array}{llll}
1 & 2.137513 & 4.735391 & -2.188690
\end{array}
$$$$
\begin{array}{llll}
6 & 0.370668 & 5.284375 & -1.067662
\end{array}
$$$$
\begin{array}{llll}
1 & 0.432770 & 6.350621 & -1.266079
\end{array}
$$$$
\begin{array}{lllll}
6 & -0.610735 & 4.761434 & -0.238958
\end{array}
$$$$
\begin{array}{llll}
1 & -1.330508 & 5.403248 & 0.260317
\end{array}
$$$$
6 \quad-0.690253 \quad 3.387855-0.011436
$$$$
6 \quad-2.541129-1.428525-2.917233
$$$$
1 \quad-3.023876 \quad-0.557801-2.478401
$$$$
\begin{array}{llll}
1 & -3.328114 & -2.052613 & -3.346548
\end{array}
$$$$
\begin{array}{llll}
1 & -1.910958 & -1.058928 & -3.732588
\end{array}
$$$$
\begin{array}{lllll}
6 & -1.734717 & 2.934990 & 0.953178
\end{array}
$$$$
\begin{array}{llll}
1 & -1.678878 & 3.548968 & 1.859102
\end{array}
$$$$
\begin{array}{llll}
1 & -1.615880 & 1.892393 & 1.220594
\end{array}
$$$$
\begin{array}{llll}
1 & -2.734418 & 3.046473 & 0.525865
\end{array}
$$$$
6 \quad-5.607197 \quad-1.020373-0.904095
$$$$
6 \quad-6.862749-0.907301-1.491816
$$$$
\begin{array}{lllll}
6 & -7.266596 & 0.308789 & -2.039962
\end{array}
$$$$
\begin{array}{lllll}
6 & -6.406275 & 1.402867 & -1.998345
\end{array}
$$$$
1 \quad-7.524418 \text { - } 1.768949-1.529936
$$$$
\begin{array}{lllll}
1 & -8.245020 & 0.400269 & -2.503766
\end{array}
$$$$
\begin{array}{lllll}
1 & -6.714398 & 2.351494 & -2.431108
\end{array}
$$$$
\begin{array}{lllll}
1 & -4.462420 & 2.128630 & -1.384174
\end{array}
$$$$
\begin{array}{lllll}
6 & -3.391541 & -0.065582 & -0.177175
\end{array}
$$$$
1 \quad-3.060773-1.107366-0.343205
$$$$
\begin{array}{llll}
6 & -3.532260 & 0.125026 & 1.330489
\end{array}
$$$$
\begin{array}{lllll}
1 & -2.551969 & 0.049678 & 1.814805
\end{array}
$$$$
\begin{array}{llll}
1 & -3.954171 & 1.108985 & 1.558748
\end{array}
$$$$
\begin{array}{lllll}
1 & -4.186331 & -0.639567 & 1.763620
\end{array}
$$$$
\begin{array}{lllll}
8 & -2.457434 & 0.857870 & -0.676461
\end{array}
$$$$
\begin{array}{lllll}
1 & -1.791047 & 0.797344 & -1.793282
\end{array}
$$$$
\begin{array}{lllll}
1 & -0.178520 & -1.658715 & 2.217780
\end{array}
$$$$
\begin{array}{lllll}
6 & -1.266577 & -2.320411 & 1.648755
\end{array}
$$$$
\begin{array}{lllll}
6 & 0.268923 & -2.071361 & 3.483915
\end{array}
$$$$
\begin{array}{lllll}
6 & -1.898046 & -3.368526 & 2.314824
\end{array}
$$$$
\begin{array}{lllll}
1 & -1.629022 & -2.003598 & 0.676673
\end{array}
$$$$
\begin{array}{lllll}
6 & -0.355486 & -3.120366 & 4.148336
\end{array}
$$$$
\begin{array}{lllll}
1 & 1.105232 & -1.555204 & 3.951308
\end{array}
$$$$
\begin{array}{lllll}
6 & -1.442757 & -3.771783 & 3.564220
\end{array}
$$$$
\begin{array}{lllll}
1 & -2.745607 & -3.864488 & 1.847195
\end{array}
$$$$
\begin{array}{lllll}
1 & 0.002117 & -3.427394 & 5.127494
\end{array}
$$$$
\begin{array}{lllll}
1 & -1.932767 & -4.587762 & 4.088468
\end{array}
$$$$
\begin{array}{lllll}
6 & 0.326952 & 1.099474 & 2.646885
\end{array}
$$$$
\begin{array}{lllll}
6 & 1.007802 & 2.322853 & 2.560834
\end{array}
$$$$
\begin{array}{lllll}
6 & -0.631583 & 0.955011 & 3.660080
\end{array}
$$$$
\begin{array}{lllll}
6 & 0.767235 & 3.346066 & 3.471747
\end{array}
$$$$
\begin{array}{lllll}
1 & 1.716213 & 2.501708 & 1.759917
\end{array}
$$$$
\begin{array}{lllll}
6 & -0.881500 & 1.984073 & 4.564094
\end{array}
$$$$
\begin{array}{lllll}
1 & -1.198057 & 0.032684 & 3.746888
\end{array}
$$$$
\begin{array}{lllll}
6 & -0.178737 & 3.181512 & 4.479958
\end{array}
$$$$
\begin{array}{lllll}
1 & 1.314356 & 4.280919 & 3.380486
\end{array}
$$$$
\begin{array}{lllll}
1 & -1.630349 & 1.842943 & 5.338697
\end{array}
$$$$
\begin{array}{llll}
1 & -0.370740 & 3.982318 & 5.188759
\end{array}
$$$$
\begin{array}{lllll}
6 & 2.374736 & -0.563006 & 1.472152
\end{array}
$$$$
\begin{array}{lllll}
6 & 2.877715 & -1.836429 & 1.769754
\end{array}
$$$$
\begin{array}{lllll}
6 & 3.293690 & 0.439469 & 1.131014
\end{array}
$$$$
\begin{array}{lllll}
6 & 4.248233 & -2.078067 & 1.784105
\end{array}
$$$$
\begin{array}{lllll}
1 & 2.196856 & -2.658348 & 1.969915
\end{array}
$$$$
\begin{array}{lllll}
6 & 4.663529 & 0.199030 & 1.144188
\end{array}
$$ 


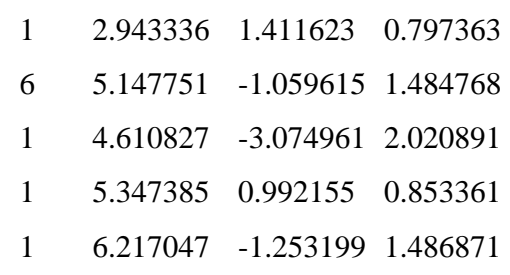

$$
\begin{aligned}
& \text { A-TS1 }_{0} \text {-PS } \\
& \begin{array}{lllll}
44 & -0.852664 & 0.023544 & -0.515238
\end{array} \\
& 1 \quad-1.492904 \quad 0.391105 \quad-1.924522 \\
& \begin{array}{llll}
15 & -2.475574 & 1.334390 & 0.288275
\end{array} \\
& \begin{array}{lllll}
7 & -2.087719 & -1.753989 & -0.368063
\end{array} \\
& \begin{array}{lllll}
7 & -0.999247 & -2.710473 & 1.616362
\end{array} \\
& \begin{array}{lllll}
7 & 0.259210 & -0.728018 & 1.118475
\end{array} \\
& \begin{array}{llll}
7 & 2.122791 & 0.720642 & 0.898037
\end{array} \\
& \begin{array}{lllll}
7 & 0.623065 & 1.603019 & -0.817356
\end{array} \\
& 6 \quad-3.039489-2.044275-1.313259 \\
& \begin{array}{lllll}
6 & -4.041219 & -2.985532 & -1.090249
\end{array} \\
& 1 \quad-4.780850-3.138707-1.869882 \\
& \begin{array}{lllll}
6 & -4.093010 & -3.706802 & 0.095788
\end{array} \\
& \begin{array}{lllll}
1 & -4.886053 & -4.424000 & 0.286142
\end{array} \\
& \begin{array}{lllll}
6 & -3.057887 & -3.534227 & 0.983492
\end{array} \\
& \begin{array}{lllll}
1 & -2.953100 & -4.126965 & 1.884907
\end{array} \\
& \begin{array}{lllll}
6 & -2.036724 & -2.596672 & 0.728164
\end{array} \\
& \begin{array}{lllll}
6 & 0.042702 & -1.953665 & 1.723816
\end{array} \\
& \begin{array}{lllll}
6 & 1.227546 & -2.313884 & 2.510659
\end{array} \\
& \begin{array}{lllll}
6 & 1.527326 & -3.409340 & 3.302379
\end{array} \\
& \begin{array}{lllll}
1 & 0.791223 & -4.190461 & 3.468208
\end{array} \\
& \begin{array}{llll}
6 & 2.804628 & -3.472381 & 3.866424
\end{array} \\
& 1 \quad 3.073014 \quad-4.316555 \quad 4.495100 \\
& \begin{array}{lllll}
6 & 3.748899 & -2.471710 & 3.626480
\end{array} \\
& \begin{array}{lllll}
1 & 4.737452 & -2.553646 & 4.069182
\end{array} \\
& \begin{array}{lllll}
6 & 3.448408 & -1.372304 & 2.817566
\end{array} \\
& \begin{array}{lllll}
1 & 4.194126 & -0.608797 & 2.611032
\end{array} \\
& \begin{array}{llll}
6 & 2.175823 & -1.311755 & 2.271591
\end{array} \\
& \begin{array}{llll}
6 & 1.509970 & -0.370384 & 1.375060
\end{array} \\
& \begin{array}{lllll}
6 & 1.769505 & 1.646766 & -0.080851
\end{array} \\
& \begin{array}{lllll}
6 & 2.766938 & 2.620283 & -0.256336
\end{array} \\
& \begin{array}{llll}
1 & 3.653571 & 2.565062 & 0.365674
\end{array} \\
& \begin{array}{lllll}
6 & 2.618071 & 3.588396 & -1.218570
\end{array} \\
& \begin{array}{lllll}
1 & 3.381904 & 4.345114 & -1.369999
\end{array} \\
& \begin{array}{llll}
6 & 1.467851 & 3.548430 & -1.997062
\end{array}
\end{aligned}
$$

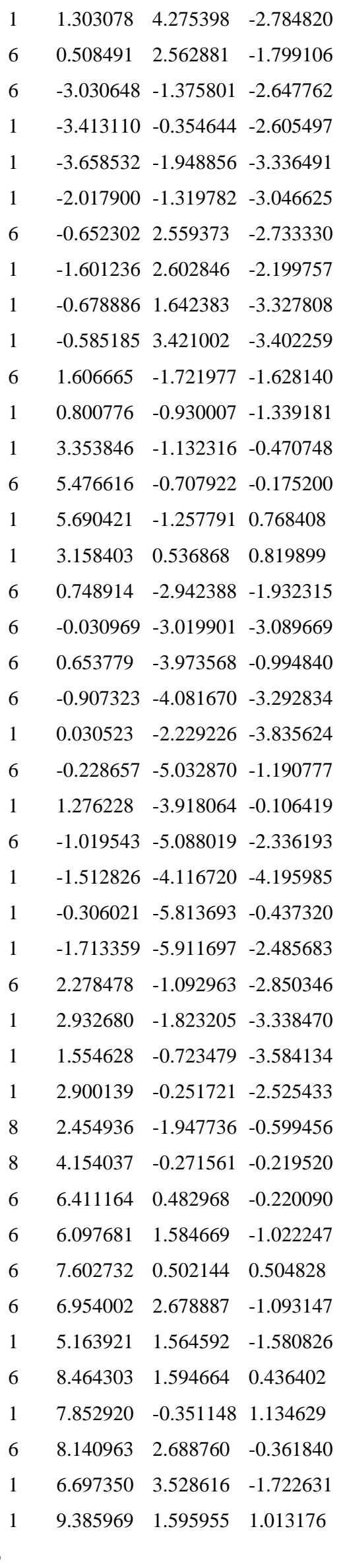




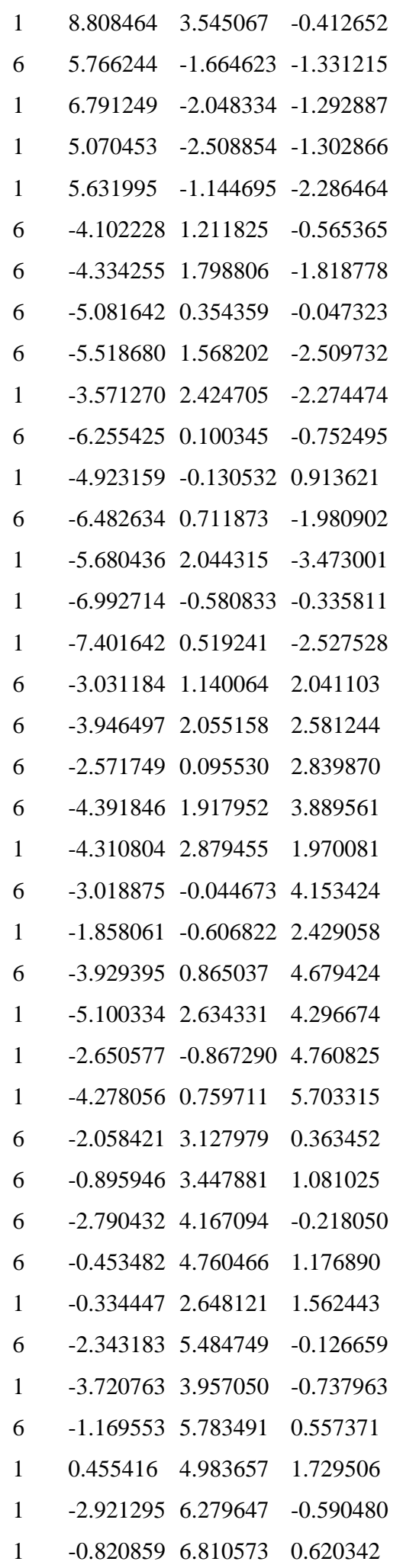

\section{A-TS1}

$44 \quad-0.154134-0.129045 \quad-0.694395$
$1 \quad-0.033501-1.399851-1.681381$

$15 \quad 1.740466 \quad-0.7199350 .532614$

$\begin{array}{lllll}7 & 1.063374 & 1.058272 & -2.006886\end{array}$

$\begin{array}{lllll}7 & 0.989915 & 3.113006 & -0.689391\end{array}$

$\begin{array}{llll}7 & -0.581555 & 1.635705 & 0.369568\end{array}$

$\begin{array}{llll}7 & -2.683356 & 0.874971 & 1.189913\end{array}$

$\begin{array}{llll}7 & -1.498008 & -1.196771 & 0.606592\end{array}$

$\begin{array}{lllll}6 & 1.504005 & 0.591124 & -3.218557\end{array}$

$\begin{array}{lllll}6 & 2.604736 & 1.141668 & -3.865789\end{array}$

$1 \quad 2.926960 \quad 0.696695 \quad-4.802456$

$\begin{array}{llll}6 & 3.260514 & 2.251342 & -3.334489\end{array}$

$1 \quad 4.135918 \quad 2.672906 \quad-3.819957$

$\begin{array}{lllll}6 & 2.701713 & 2.845485 & -2.229040\end{array}$

$\begin{array}{lllll}1 & 3.072778 & 3.774377 & -1.811069\end{array}$

$\begin{array}{lllll}6 & 1.576179 & 2.275891 & -1.599360\end{array}$

$\begin{array}{llll}6 & -0.016169 & 2.873285 & 0.083045\end{array}$

$\begin{array}{llll}6 & -0.871462 & 3.921850 & 0.646220\end{array}$

$\begin{array}{llll}6 & -0.757292 & 5.300395 & 0.707492\end{array}$

$\begin{array}{llll}1 & 0.130548 & 5.798788 & 0.328665\end{array}$

$\begin{array}{llll}6 & -1.818083 & 6.017824 & 1.268004\end{array}$

$\begin{array}{llll}1 & -1.758138 & 7.100464 & 1.334177\end{array}$

$\begin{array}{llll}6 & -2.960903 & 5.364971 & 1.738708\end{array}$

$1 \quad-3.773005 \quad 5.951168 \quad 2.159881$

$\begin{array}{llll}6 & -3.077686 & 3.974887 & 1.674333\end{array}$

$\begin{array}{llll}1 & -3.968113 & 3.467105 & 2.034760\end{array}$

$\begin{array}{llll}6 & -2.018860 & 3.264218 & 1.124213\end{array}$

$\begin{array}{llll}6 & -1.763130 & 1.846377 & 0.923714\end{array}$

$\begin{array}{lllll}6 & -2.436545 & -0.509804 & 1.323815\end{array}$

$\begin{array}{lllll}6 & -3.310889 & -1.127671 & 2.219978\end{array}$

$\begin{array}{lllll}1 & -4.004457 & -0.494439 & 2.762163\end{array}$

$6 \quad-3.262087 \quad-2.4953992 .406403$

$1 \quad-3.924789-2.990554 \quad 3.109817$

$\begin{array}{lllll}6 & -2.340803 & -3.207860 & 1.657328\end{array}$

$\begin{array}{lllll}1 & -2.268034 & -4.287491 & 1.738808\end{array}$

$\begin{array}{llll}6 & -1.488128 & -2.556640 & 0.768326\end{array}$

$\begin{array}{lllll}6 & 0.758265 & -0.508790 & -3.900334\end{array}$

$11.043987 \quad-1.501867 \quad-3.543405$

$1 \quad 0.949545 \quad-0.463226 \quad-4.976895$

$1 \quad-0.312992 \quad-0.413518-3.704507$

$6 \quad-0.570743 \quad-3.394993 \quad-0.050587$

$1 \quad 0.480201 \quad-3.1446200 .112109$

$1 \quad-0.757792 \quad-3.231912-1.115649$

$\begin{array}{lllll}1 & -0.717300 & -4.450976 & 0.191777\end{array}$ 


\begin{tabular}{|c|c|c|c|}
\hline 6 & -3.123775 & 1.115671 & -3.167076 \\
\hline 6 & -3.447546 & 0.513488 & -1.820389 \\
\hline 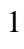 & -1.692529 & 0.219288 & -1.547805 \\
\hline & -3.881939 & 1.271531 & -0.920934 \\
\hline 1 & -3.464805 & 1.027895 & 0.566556 \\
\hline & -2.550810 & 0.445220 & -3.810326 \\
\hline 1 & -4.069751 & 1.349893 & -3.671964 \\
\hline 1 & -2.570148 & 2.046422 & -3.031868 \\
\hline 6 & -3.769435 & -0.951771 & -1.756353 \\
\hline 6 & -3.092772 & -1.914038 & -2.514662 \\
\hline 6 & -4.801560 & -1.364771 & -0.909929 \\
\hline 6 & -3.440936 & -3.255236 & -2.424992 \\
\hline 1 & -2.247100 & -1.608973 & -3.127223 \\
\hline 6 & -5.151574 & -2.708827 & -0.818031 \\
\hline 1 & -5.325230 & -0.609721 & -0.328692 \\
\hline b & -4.471008 & -3.658150 & -1.573945 \\
\hline 1 & -2.896589 & -3.994373 & -3.007536 \\
\hline 1 & -5.953928 & -3.014397 & -0.150932 \\
\hline 1 & -4.738365 & -4.709222 & -1.501430 \\
\hline 6 & 2.948293 & -1.964737 & -0.099423 \\
\hline 6 & 4.155475 & -2.226890 & 0.566056 \\
\hline 6 & 2.671863 & -2.654255 & -1.281728 \\
\hline 6 & 5.052833 & -3.162625 & 0.064068 \\
\hline 1 & 4.391076 & -1.688381 & 1.482365 \\
\hline 6 & 3.572274 & -3.589894 & -1.788781 \\
\hline 1 & 1.730386 & -2.441173 & -1.787032 \\
\hline 6 & 4.762446 & -3.846477 & -1.116330 \\
\hline 1 & 5.982716 & -3.357551 & 0.592090 \\
\hline 1 & 3.341544 & -4.117652 & -2.710808 \\
\hline 1 & 5.466422 & -4.574830 & -1.509937 \\
\hline 6 & 1.304152 & -1.390932 & 2.197190 \\
\hline 6 & 0.389127 & -0.663647 & 2.974954 \\
\hline 6 & 1.734155 & -2.633718 & 2.676679 \\
\hline 6 & -0.046047 & -1.138793 & 4.206371 \\
\hline 1 & -0.013297 & 0.270158 & 2.585430 \\
\hline 6 & 1.288054 & -3.117035 & 3.905276 \\
\hline 1 & 2.414148 & -3.238780 & 2.083375 \\
\hline 6 & 0.406087 & -2.369167 & 4.678473 \\
\hline 1 & -0.755469 & -0.555116 & 4.787728 \\
\hline 1 & 1.634479 & -4.085483 & 4.256959 \\
\hline 1 & 0.060163 & -2.748406 & 5.636179 \\
\hline 6 & 2.906215 & 0.656040 & 0.936929 \\
\hline 5 & 2.637543 & 1.569811 & 1.964390 \\
\hline
\end{tabular}

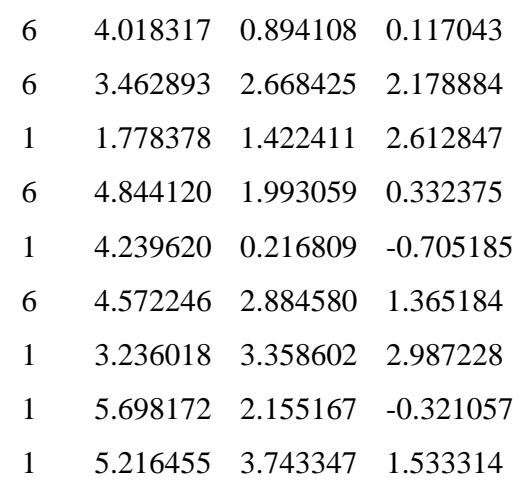

A-2 0

$\begin{array}{lllll}44 & 0.422218 & -0.032649 & -1.475846\end{array}$

$\begin{array}{llll}1 & 0.387867 & 0.054355 & -3.121001\end{array}$

$\begin{array}{lllll}15 & 0.957594 & -0.115844 & 0.891270\end{array}$

$\begin{array}{lllll}7 & 0.557872 & 2.131543 & -1.623764\end{array}$

$\begin{array}{lllll}7 & -1.657944 & 2.497645 & -0.735118\end{array}$

$\begin{array}{lllll}7 & -1.621966 & 0.165393 & -1.073241\end{array}$

$7 \quad-2.166175 \quad-2.160156-0.784868$

$\begin{array}{lllll}7 & 0.132374 & -2.113615 & -1.664614\end{array}$

$\begin{array}{lllll}6 & 1.634746 & 2.756141 & -2.208906\end{array}$

$\begin{array}{lllll}6 & 1.813352 & 4.134299 & -2.163698\end{array}$

$\begin{array}{lllll}1 & 2.701738 & 4.554997 & -2.622734\end{array}$

$\begin{array}{lllll}6 & 0.880368 & 4.948274 & -1.530853\end{array}$

$1 \quad 1.023272 \quad 6.021636 \quad-1.455720$

$\begin{array}{lllll}6 & -0.257108 & 4.351872 & -1.039833\end{array}$

$\begin{array}{lllll}1 & -1.053080 & 4.937944 & -0.588541\end{array}$

$\begin{array}{lllll}6 & -0.411830 & 2.959264 & -1.146385\end{array}$

$\begin{array}{lllll}6 & -2.265505 & 1.276195 & -0.767833\end{array}$

$\begin{array}{llll}6 & -3.629632 & 1.031173 & -0.345418\end{array}$

$\begin{array}{llll}6 & -4.684322 & 1.839247 & 0.069667\end{array}$

$1 \quad-4.605896 \quad 2.924434 \quad 0.099710$

$\begin{array}{llll}6 & -5.874402 & 1.214792 & 0.451673\end{array}$

$\begin{array}{llll}1 & -6.715681 & 1.822783 & 0.772526\end{array}$

$\begin{array}{llll}6 & -6.002297 & -0.176338 & 0.428225\end{array}$

$\begin{array}{llll}1 & -6.940512 & -0.629912 & 0.734764\end{array}$

$\begin{array}{llll}6 & -4.939199 & -0.987224 & 0.019095\end{array}$

$\begin{array}{llll}1 & -5.016980 & -2.070637 & 0.012672\end{array}$

$\begin{array}{lllll}6 & -3.763694 & -0.372194 & -0.373519\end{array}$

$\begin{array}{lllll}6 & -2.464372 & -0.900615 & -0.790889\end{array}$

$6 \quad-0.994923-2.751725-1.162118$

$6 \quad-1.038435-4.156043-1.019240$ 


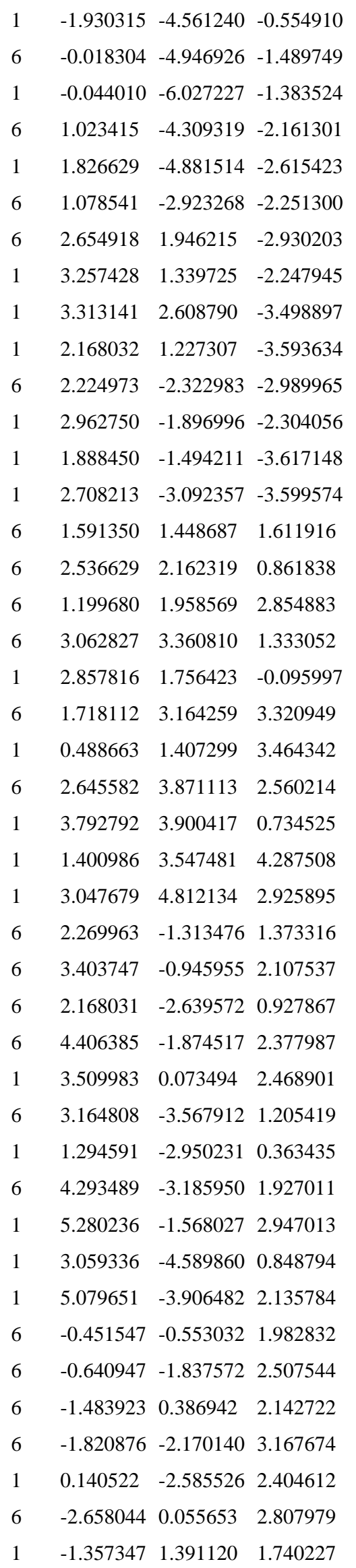

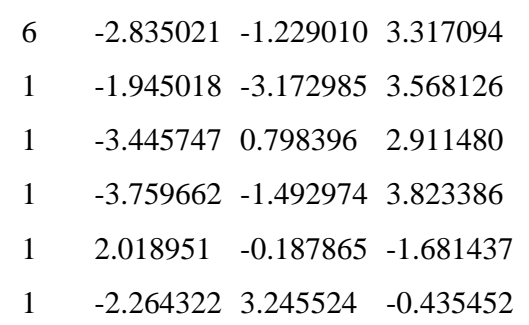

$\mathrm{A}^{-T S 2} 2_{0}$

$\begin{array}{lllll}44 & 0.169355 & 0.247110 & -1.279359\end{array}$

$\begin{array}{lllll}1 & -1.067377 & 0.554931 & -2.286899\end{array}$

$\begin{array}{llll}15 & -1.403404 & 0.216988 & 0.399803\end{array}$

$7 \quad-0.013846-1.900510-1.421516$

$\begin{array}{lllll}7 & 1.666754 & -2.494957 & 0.267476\end{array}$

$\begin{array}{lllll}7 & 1.966501 & -0.197627 & -0.315534\end{array}$

$\begin{array}{lllll}7 & 2.943369 & 1.523168 & -1.535775\end{array}$

$\begin{array}{lllll}7 & 0.714390 & 2.355005 & -1.212774\end{array}$

$6 \quad-0.820711-2.468844-2.367804$

$6 \quad-1.303130 \quad-3.768716 \quad-2.234567$

$1 \quad-1.969558-4.154153-3.000419$

$6 \quad-0.920150-4.556313-1.153327$

$1 \quad-1.314003-5.559958-1.022250$

$\begin{array}{lllll}6 & 0.037180 & -4.051638 & -0.301322\end{array}$

$\begin{array}{lllll}1 & 0.478418 & -4.636742 & 0.497384\end{array}$

$\begin{array}{lllll}6 & 0.528205 & -2.744467 & -0.472867\end{array}$

$\begin{array}{lllll}6 & 2.360244 & -1.405794 & 0.244354\end{array}$

$\begin{array}{lllll}6 & 3.796268 & -1.319012 & 0.545694\end{array}$

$\begin{array}{lllll}6 & 4.683929 & -2.184623 & 1.159873\end{array}$

$\begin{array}{lllll}1 & 4.333700 & -3.109731 & 1.609125\end{array}$

$\begin{array}{lllll}6 & 6.036968 & -1.829770 & 1.180238\end{array}$

$\begin{array}{llll}1 & 6.756163 & -2.481078 & 1.668750\end{array}$

$\begin{array}{lllll}6 & 6.479950 & -0.654002 & 0.569389\end{array}$

$\begin{array}{lllll}1 & 7.539056 & -0.411988 & 0.588077\end{array}$

$\begin{array}{lllll}6 & 5.588958 & 0.210363 & -0.071536\end{array}$

$\begin{array}{llll}1 & 5.933232 & 1.114672 & -0.565607\end{array}$

$\begin{array}{lllll}6 & 4.241555 & -0.127298 & -0.069058\end{array}$

$\begin{array}{lllll}6 & 3.051962 & 0.516504 & -0.605097\end{array}$

$\begin{array}{lllll}6 & 2.064507 & 2.579664 & -1.230283\end{array}$

$\begin{array}{lllll}6 & 2.611658 & 3.847319 & -1.041567\end{array}$

$1 \quad 3.691500 \quad 3.938520 \quad-1.060462$

$\begin{array}{lllll}6 & 1.773792 & 4.934797 & -0.860876\end{array}$

$\begin{array}{lllll}1 & 2.179167 & 5.930733 & -0.706821\end{array}$ S 19 


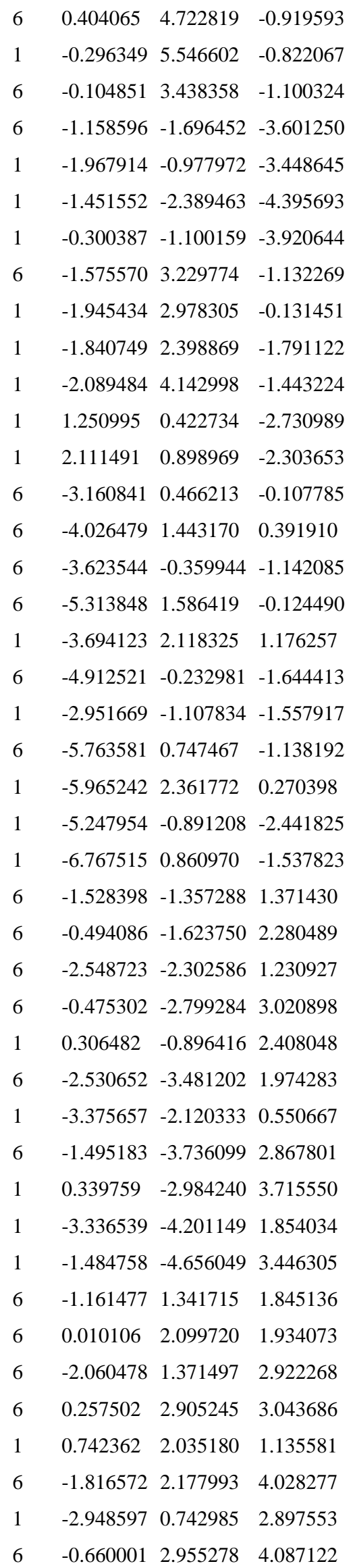

\section{A-TS2 $2_{0}-\mathrm{PS}$}

$1 \quad 2.071676 \quad 0.667625 \quad 1.953286$

$15 \quad 2.833950 \quad-0.697318-0.289273$

$\begin{array}{llll}7 & 0.334103 & -1.500898 & 2.049690\end{array}$

$7 \quad-1.584415-1.969423 \quad 0.644159$

$\begin{array}{lllll}7 & -0.399900 & -0.365732 & -0.626144\end{array}$

$\begin{array}{llll}7 & 0.076682 & 1.289358 & -2.295553\end{array}$

$\begin{array}{lllll}7 & 1.255150 & 2.041069 & -0.272043\end{array}$

$\begin{array}{llll}6 & 0.947918 & -1.810984 & 3.235413\end{array}$

$\begin{array}{llll}6 & 0.286107 & -2.474540 & 4.260483\end{array}$

$1 \quad 0.827446 \quad-2.683615 \quad 5.177252$

$\begin{array}{llll}6 & -1.042810 & -2.853502 & 4.106162\end{array}$

$\begin{array}{llll}1 & -1.586843 & -3.334892 & 4.912935\end{array}$

$\begin{array}{llll}6 & -1.644411 & -2.633468 & 2.889382\end{array}$

$\begin{array}{llll}1 & -2.678028 & -2.897588 & 2.691110\end{array}$

$6 \quad-0.923918-1.985855 \quad 1.872155$

$6 \quad-1.381961-1.233186-0.462288$

$6 \quad-2.249123-1.292232-1.633761$

$6 \quad-3.381088-2.030478-1.948567$

$1 \quad-3.816829 \quad-2.734778 \quad-1.244854$

$6 \quad-3.976020 \quad-1.799334 \quad-3.190119$

$1 \quad-4.878227-2.341341-3.457642$

$6 \quad-3.452935-0.855885-4.076545$

$1 \quad-3.950576-0.689734-5.028100$

$6 \quad-2.317266 \quad-0.108810 \quad-3.751859$

$1 \quad-1.912453 \quad 0.637911 \quad-4.428751$

$6 \quad-1.721843 \quad-0.343619-2.523851$

$\begin{array}{lllll}6 & -0.576140 & 0.270440 & -1.838174\end{array}$

$\begin{array}{llll}6 & 0.938507 & 2.113708 & -1.613451\end{array}$

$6 \quad 1.413379 \quad 3.172889-2.411752$

$1 \quad 1.164615 \quad 3.132372 \quad-3.465803$

$\begin{array}{lllll}6 & 2.108090 & 4.219628 & -1.851479\end{array}$

$1 \quad 2.482867 \quad 5.035865 \quad-2.462445$

$\begin{array}{llll}6 & 2.247477 & 4.231760 & -0.468112\end{array}$

$\begin{array}{llll}1 & 2.694292 & 5.078418 & 0.043559\end{array}$

$\begin{array}{lllll}6 & 1.794423 & 3.162056 & 0.299398\end{array}$

$\begin{array}{llll}6 & 2.382995 & -1.462293 & 3.429388\end{array}$

S 20 


$$
\begin{aligned}
& 1 \quad 2.965594 \quad-1.682543 \quad 2.532311 \\
& \begin{array}{llll}
1 & 2.792773 & -2.034338 & 4.265736
\end{array} \\
& \begin{array}{llll}
1 & 2.526747 & -0.397289 & 3.628312
\end{array} \\
& \begin{array}{lllll}
6 & 1.836727 & 3.296427 & 1.785181
\end{array} \\
& \begin{array}{llll}
1 & 2.501150 & 2.575072 & 2.265217
\end{array} \\
& \begin{array}{llll}
1 & 0.837241 & 3.127116 & 2.202068
\end{array} \\
& \begin{array}{llll}
1 & 2.145714 & 4.310296 & 2.051567
\end{array} \\
& \begin{array}{llll}
44 & 1.009828 & 0.223397 & 0.828860
\end{array} \\
& \begin{array}{llll}
1 & 0.079301 & 1.113143 & 2.028648
\end{array} \\
& \begin{array}{llll}
1 & -2.590242 & -2.200470 & 0.751876
\end{array} \\
& \begin{array}{llll}
8 & -2.608649 & 0.447109 & 1.553859
\end{array} \\
& 1 \quad-3.485073 \quad-0.549857 \quad 1.509835 \\
& \begin{array}{llll}
8 & -4.067855 & -1.495959 & 1.370122
\end{array} \\
& \begin{array}{llll}
1 & -0.562669 & 0.820761 & 1.565868
\end{array} \\
& \begin{array}{llll}
6 & -2.975751 & 1.470666 & 2.405354
\end{array} \\
& \begin{array}{llll}
1 & -4.078748 & 1.646297 & 2.363287
\end{array} \\
& 6 \quad-5.450162 \quad-1.316238 \quad 1.198140 \\
& 1 \quad-5.821130 \quad-0.6368591 .992751 \\
& 6 \quad-5.792951-0.647876-0.128993 \\
& 6 \quad-6.876068-1.033994-0.923614 \\
& \begin{array}{llll}
6 & -5.000225 & 0.421988 & -0.567468
\end{array} \\
& 6 \quad-7.159645-0.373659-2.119944 \\
& 1 \quad-7.512281-1.861376-0.617257 \\
& 6 \quad-5.283154 \quad 1.082381 \quad-1.755832 \\
& 1 \quad-4.1272090 .705065 \quad 0.019620 \\
& \begin{array}{llll}
6 & -6.366179 & 0.688502 & -2.540240
\end{array} \\
& \begin{array}{llll}
1 & -8.006444 & -0.693742 & -2.722972
\end{array} \\
& \begin{array}{llll}
1 & -4.644758 & 1.903727 & -2.076194
\end{array} \\
& \begin{array}{llll}
1 & -6.586642 & 1.202203 & -3.473179
\end{array} \\
& \begin{array}{llll}
6 & -2.345030 & 2.791264 & 1.968500
\end{array} \\
& \begin{array}{lllll}
6 & -2.164233 & 3.875140 & 2.835167
\end{array} \\
& \begin{array}{llll}
6 & -1.944375 & 2.946388 & 0.635422
\end{array} \\
& \begin{array}{llll}
6 & -1.597517 & 5.068220 & 2.389207
\end{array} \\
& \begin{array}{llll}
1 & -2.467122 & 3.790834 & 3.876644
\end{array} \\
& \begin{array}{llll}
6 & -1.386596 & 4.138617 & 0.183475
\end{array} \\
& \begin{array}{llll}
1 & -2.059509 & 2.094698 & -0.032732
\end{array} \\
& \begin{array}{llll}
6 & -1.204382 & 5.206000 & 1.060323
\end{array} \\
& \begin{array}{llll}
1 & -1.460758 & 5.892185 & 3.086084
\end{array} \\
& \begin{array}{lllll}
1 & -1.075437 & 4.226639 & -0.856828
\end{array} \\
& \begin{array}{llll}
1 & -0.757219 & 6.134048 & 0.711182
\end{array} \\
& \begin{array}{llll}
6 & -6.133622 & -2.657349 & 1.400833
\end{array} \\
& \begin{array}{llll}
1 & -5.811300 & -3.085850 & 2.352770
\end{array} \\
& \begin{array}{llll}
1 & -7.224303 & -2.573058 & 1.418250
\end{array}
\end{aligned}
$$

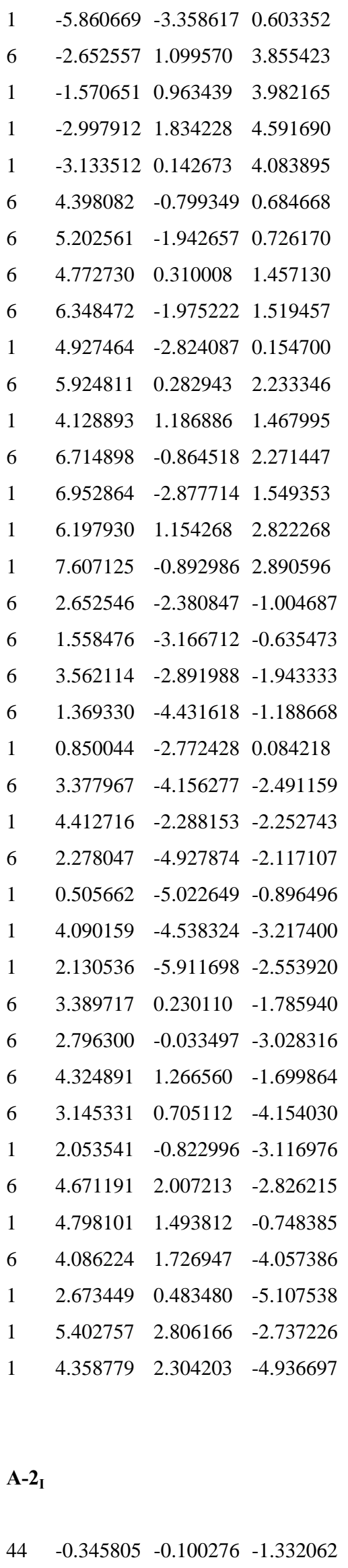


$1 \quad-1.913571 \quad-0.450654-1.588719$

$\begin{array}{llll}15 & -1.017492 & -0.207303 & 0.755419\end{array}$

$\begin{array}{lllll}7 & 0.274075 & -2.145377 & -1.601625\end{array}$

$7 \quad 2.531672 \quad-1.858939-0.672653$

$\begin{array}{llll}7 & 1.668842 & 0.361095 & -1.017467\end{array}$

$\begin{array}{llll}7 & 1.434337 & 2.723781 & -0.606796\end{array}$

$\begin{array}{lllll}7 & -0.691020 & 1.997297 & -1.594931\end{array}$

$6 \quad-0.518960-3.051384-2.266397$

$6 \quad-0.228292 \quad-4.407869-2.301608$

$\begin{array}{lllll}1 & -0.902262 & -5.068314 & -2.838137\end{array}$

$6 \quad 0.896016 \quad-4.904971-1.641971$

$\begin{array}{lllll}1 & 1.107812 & -5.970127 & -1.620447\end{array}$

$6 \quad 1.752106 \quad-3.999242-1.066861$

$1 \quad 2.684104 \quad-4.291023-0.596430$

$6 \quad 1.485712 \quad-2.610100 \quad-1.114751$

$\begin{array}{lllll}6 & 2.624522 & -0.556855 & -0.685593\end{array}$

$\begin{array}{llll}6 & 3.826175 & 0.148771 & -0.221875\end{array}$

$\begin{array}{lllll}6 & 5.064725 & -0.299073 & 0.211151\end{array}$

$\begin{array}{llll}1 & 5.298564 & -1.359990 & 0.216600\end{array}$

$\begin{array}{lllll}6 & 5.983885 & 0.658110 & 0.646736\end{array}$

$\begin{array}{llll}1 & 6.966526 & 0.343430 & 0.988323\end{array}$

$\begin{array}{llll}6 & 5.659961 & 2.018918 & 0.655365\end{array}$

$\begin{array}{llll}1 & 6.396460 & 2.738519 & 1.002969\end{array}$

$\begin{array}{llll}6 & 4.406492 & 2.463919 & 0.228512\end{array}$

$\begin{array}{llll}1 & 4.138302 & 3.516511 & 0.242148\end{array}$

$\begin{array}{lllll}6 & 3.502109 & 1.510333 & -0.213786\end{array}$

$\begin{array}{lllll}6 & 2.109929 & 1.606170 & -0.670995\end{array}$

$\begin{array}{lllll}6 & 0.159979 & 2.937373 & -1.042814\end{array}$

$\begin{array}{llll}6 & -0.243859 & 4.288510 & -0.933093\end{array}$

$\begin{array}{llll}1 & 0.435764 & 4.953655 & -0.412838\end{array}$

$\begin{array}{lllll}6 & -1.404961 & 4.726872 & -1.519806\end{array}$

$1 \quad-1.708687 \quad 5.767650 \quad-1.453234$

$\begin{array}{lllll}6 & -2.148959 & 3.806308 & -2.258097\end{array}$

$\begin{array}{llll}1 & -3.027766 & 4.112933 & -2.816775\end{array}$

$\begin{array}{llll}6 & -1.784443 & 2.467528 & -2.276295\end{array}$

$6 \quad-1.761714-2.589696-2.954586$

$\begin{array}{lllll}1 & -2.546343 & -2.335747 & -2.235077\end{array}$

$1 \quad-2.130156-3.379920-3.615027$

$1 \quad-1.590260 \quad-1.682009-3.538793$

$\begin{array}{lllll}6 & -2.613024 & 1.524735 & -3.088469\end{array}$

$\begin{array}{lllll}1 & -3.250609 & 0.889068 & -2.467727\end{array}$

$\begin{array}{llll}1 & -1.986019 & 0.837422 & -3.663213\end{array}$

$\begin{array}{llll}1 & -3.246630 & 2.091331 & -3.777035\end{array}$ $\begin{array}{llll}6 & -1.873672 & 1.305070 & 1.347083\end{array}$

$\begin{array}{lllll}6 & -2.966237 & 1.726193 & 0.577355\end{array}$

$\begin{array}{llll}6 & -1.544305 & 2.026295 & 2.499330\end{array}$

$\begin{array}{llll}6 & -3.701396 & 2.847921 & 0.936450\end{array}$

$1 \quad-3.230141 \quad 1.153712 \quad-0.310116$

$\begin{array}{llll}6 & -2.275131 & 3.160488 & 2.851353\end{array}$

$\begin{array}{llll}1 & -0.725260 & 1.698962 & 3.133234\end{array}$

$\begin{array}{llll}6 & -3.348969 & 3.576700 & 2.070843\end{array}$

$1 \quad-4.542861 \quad 3.160724 \quad 0.323413$

$1 \quad-2.003834 \quad 3.715728 \quad 3.745270$

$\begin{array}{llll}1 & -3.914602 & 4.461598 & 2.349579\end{array}$

$\begin{array}{lllll}6 & 0.378699 & -0.497778 & 1.909021\end{array}$

$\begin{array}{lllll}6 & 0.654535 & -1.785463 & 2.385563\end{array}$

$\begin{array}{llll}6 & 1.304867 & 0.525486 & 2.165893\end{array}$

$\begin{array}{lllll}6 & 1.822096 & -2.043377 & 3.097386\end{array}$

$\begin{array}{llll}1 & -0.041504 & -2.597418 & 2.189282\end{array}$

$\begin{array}{lllll}6 & 2.465718 & 0.266530 & 2.885558\end{array}$

$\begin{array}{llll}1 & 1.127648 & 1.526767 & 1.778501\end{array}$

$\begin{array}{llll}6 & 2.731083 & -1.020196 & 3.348322\end{array}$

$1 \quad 2.022674 \quad-3.051213 \quad 3.451194$

$\begin{array}{llll}1 & 3.178060 & 1.069044 & 3.060549\end{array}$

$1 \quad 3.648787 \quad-1.224267 \quad 3.893363$

$\begin{array}{lllll}6 & -2.223017 & -1.505908 & 1.266440\end{array}$

$\begin{array}{llll}6 & -2.160643 & -2.773006 & 0.676922\end{array}$

$\begin{array}{lllll}6 & -3.176645 & -1.279793 & 2.266541\end{array}$

$6 \quad-3.027760 \quad-3.787711 \quad 1.067083$

$1 \quad-1.416010-2.965828-0.086773$

$\begin{array}{lllll}6 & -4.049759 & -2.293808 & 2.652600\end{array}$

$\begin{array}{lllll}1 & -3.243352 & -0.306212 & 2.745594\end{array}$

$\begin{array}{llll}6 & -3.980626 & -3.548791 & 2.054115\end{array}$

$1 \quad-2.956661-4.764577 \quad 0.594955$

$1 \quad-4.788012 \quad-2.099744 \quad 3.426141$

$1 \quad-4.665119 \quad-4.336544 \quad 2.357214$

\section{A-TS1_-PS}

$44 \quad-0.345805-0.100276-1.332062$

$\begin{array}{llll}1 & -1.913571 & -0.450654 & -1.588719\end{array}$

$\begin{array}{llll}15 & -1.017492 & -0.207303 & 0.755419\end{array}$

$7 \quad 0.274075 \quad-2.145377 \quad-1.601625$

$7 \quad 2.531672 \quad-1.858939-0.672653$

$\begin{array}{llll}7 & 1.668842 & 0.361095 & -1.017467\end{array}$ 
$$
\begin{array}{lllll}
6 & -0.518960 & -3.051384 & -2.266397
\end{array}
$$$$
6 \quad-0.228292-4.407869-2.301608
$$$$
\begin{array}{lllll}
1 & -0.902262 & -5.068314 & -2.838137
\end{array}
$$$$
\begin{array}{lllll}
6 & 0.896016 & -4.904971 & -1.641971
\end{array}
$$$$
\begin{array}{lllll}
1 & 1.107812 & -5.970127 & -1.620447
\end{array}
$$$$
6 \quad 1.752106 \quad-3.999242 \quad-1.066861
$$$$
1 \quad 2.684104 \quad-4.291023 \quad-0.596430
$$$$
\begin{array}{lllll}
6 & 1.485712 & -2.610100 & -1.114751
\end{array}
$$$$
\begin{array}{lllll}
6 & 2.624522 & -0.556855 & -0.685593
\end{array}
$$$$
\begin{array}{lllll}
6 & 3.826175 & 0.148771 & -0.221875
\end{array}
$$$$
\begin{array}{lllll}
6 & 5.064725 & -0.299073 & 0.211151
\end{array}
$$$$
\begin{array}{lllll}
1 & 5.298564 & -1.359990 & 0.216600
\end{array}
$$$$
\begin{array}{lllll}
6 & 5.983885 & 0.658110 & 0.646736
\end{array}
$$$$
\begin{array}{llll}
1 & 6.966526 & 0.343430 & 0.988323
\end{array}
$$$$
\begin{array}{llll}
6 & 5.659961 & 2.018918 & 0.655365
\end{array}
$$$$
\begin{array}{llll}
1 & 6.396460 & 2.738519 & 1.002969
\end{array}
$$$$
\begin{array}{llll}
6 & 4.406492 & 2.463919 & 0.228512
\end{array}
$$$$
\begin{array}{llll}
1 & 4.138302 & 3.516511 & 0.242148
\end{array}
$$$$
\begin{array}{lllll}
6 & 3.502109 & 1.510333 & -0.213786
\end{array}
$$$$
\begin{array}{lllll}
6 & 2.109929 & 1.606170 & -0.670995
\end{array}
$$$$
\begin{array}{lllll}
6 & 0.159979 & 2.937373 & -1.042814
\end{array}
$$$$
\begin{array}{lllll}
6 & -0.243859 & 4.288510 & -0.933093
\end{array}
$$$$
\begin{array}{lllll}
1 & 0.435764 & 4.953655 & -0.412838
\end{array}
$$$$
\begin{array}{llll}
6 & -1.404961 & 4.726872 & -1.519806
\end{array}
$$$$
1 \quad-1.708687 \quad 5.767650 \quad-1.453234
$$$$
\begin{array}{lllll}
6 & -2.148959 & 3.806308 & -2.258097
\end{array}
$$$$
\begin{array}{lllll}
1 & -3.027766 & 4.112933 & -2.816775
\end{array}
$$$$
\begin{array}{lllll}
6 & -1.784443 & 2.467528 & -2.276295
\end{array}
$$$$
6 \quad-1.761714 \quad-2.589696-2.954586
$$$$
\begin{array}{lllll}
1 & -2.546343 & -2.335747 & -2.235077
\end{array}
$$$$
1 \quad-2.130156 \quad-3.379920-3.615027
$$$$
1 \quad-1.590260-1.682009-3.538793
$$$$
\begin{array}{lllll}
6 & -2.613024 & 1.524735 & -3.088469
\end{array}
$$$$
\begin{array}{lllll}
1 & -3.250609 & 0.889068 & -2.467727
\end{array}
$$$$
\begin{array}{llll}
1 & -1.986019 & 0.837422 & -3.663213
\end{array}
$$$$
\begin{array}{llll}
1 & -3.246630 & 2.091331 & -3.777035
\end{array}
$$$$
\begin{array}{llll}
6 & -1.873672 & 1.305070 & 1.347083
\end{array}
$$$$
\begin{array}{lllll}
6 & -2.966237 & 1.726193 & 0.577355
\end{array}
$$$$
\begin{array}{lllll}
6 & -1.544305 & 2.026295 & 2.499330
\end{array}
$$$$
\begin{array}{llll}
6 & -3.701396 & 2.847921 & 0.936450
\end{array}
$$$$
1 \quad-3.230141 \quad 1.153712 \quad-0.310116
$$

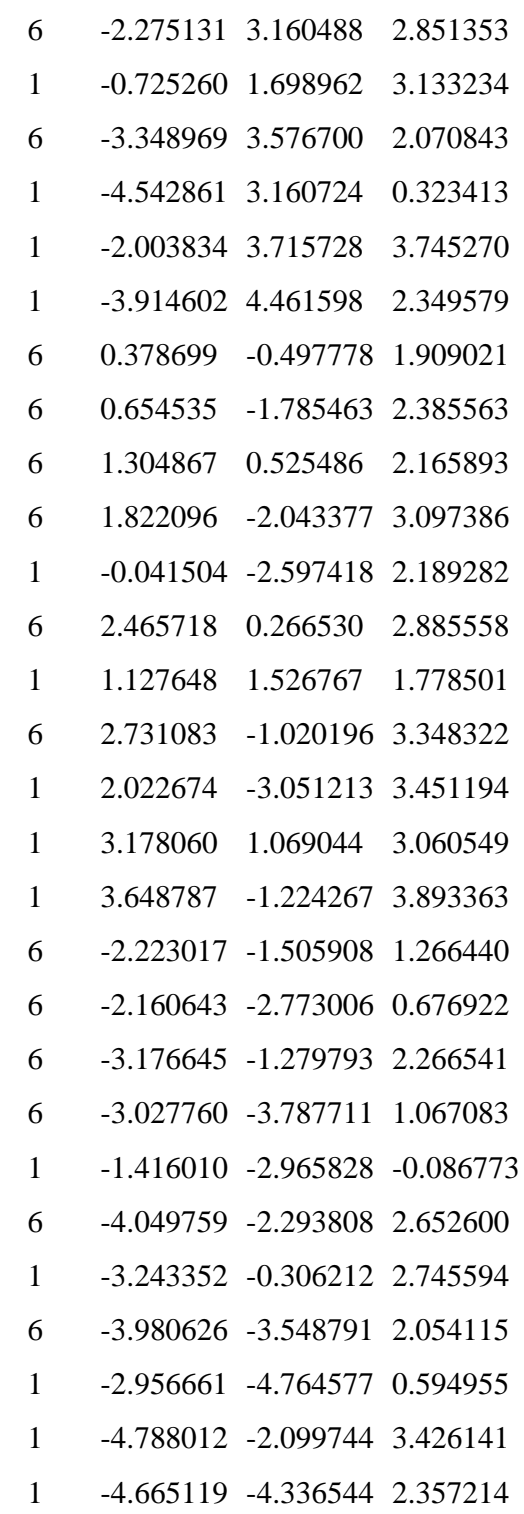

\section{A-TS1}

$44 \quad-0.345805-0.100276-1.332062$

$\begin{array}{lllll}1 & -1.913571 & -0.450654 & -1.588719\end{array}$

$\begin{array}{lllll}15 & -1.017492 & -0.207303 & 0.755419\end{array}$

$\begin{array}{lllll}7 & 0.274075 & -2.145377 & -1.601625\end{array}$

$\begin{array}{lllll}7 & 2.531672 & -1.858939 & -0.672653\end{array}$

$\begin{array}{lllll}7 & 1.668842 & 0.361095 & -1.017467\end{array}$

$\begin{array}{lllll}7 & 1.434337 & 2.723781 & -0.606796\end{array}$

$\begin{array}{lllll}7 & -0.691020 & 1.997297 & -1.594931\end{array}$

$\begin{array}{lllll}6 & -0.518960 & -3.051384 & -2.266397\end{array}$

$6 \quad-0.228292-4.407869-2.301608$

$\begin{array}{llll}1 & -0.902262 & -5.068314 & -2.838137\end{array}$ 


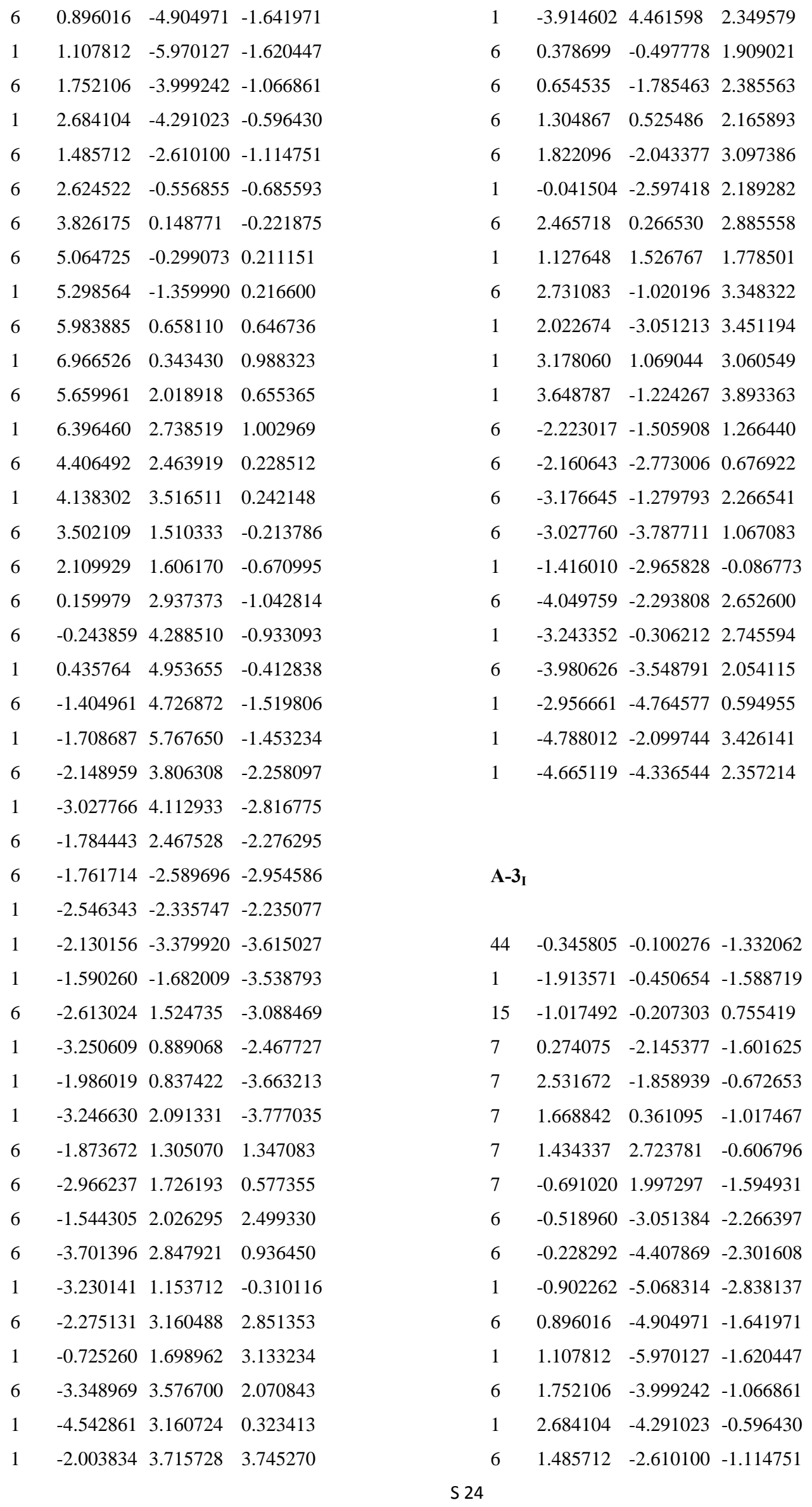




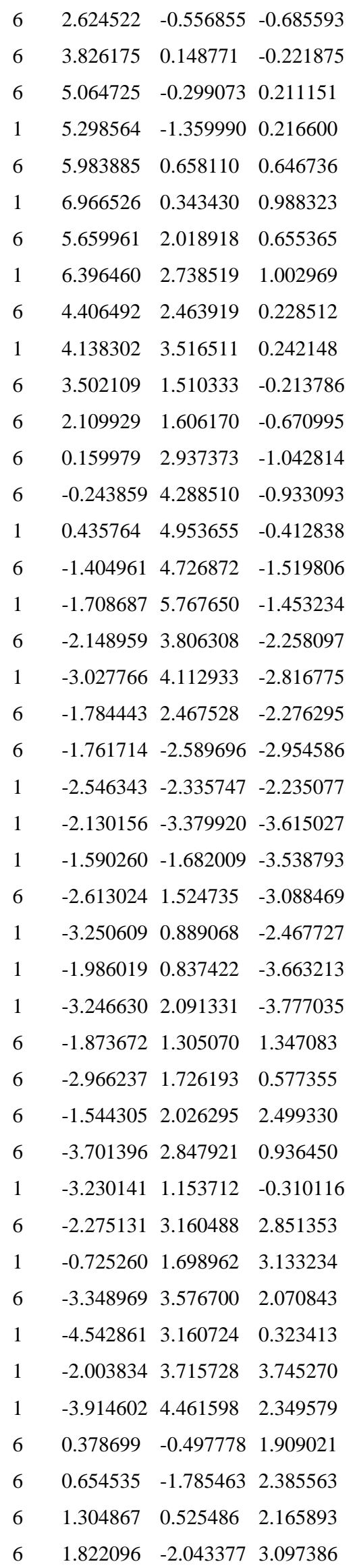




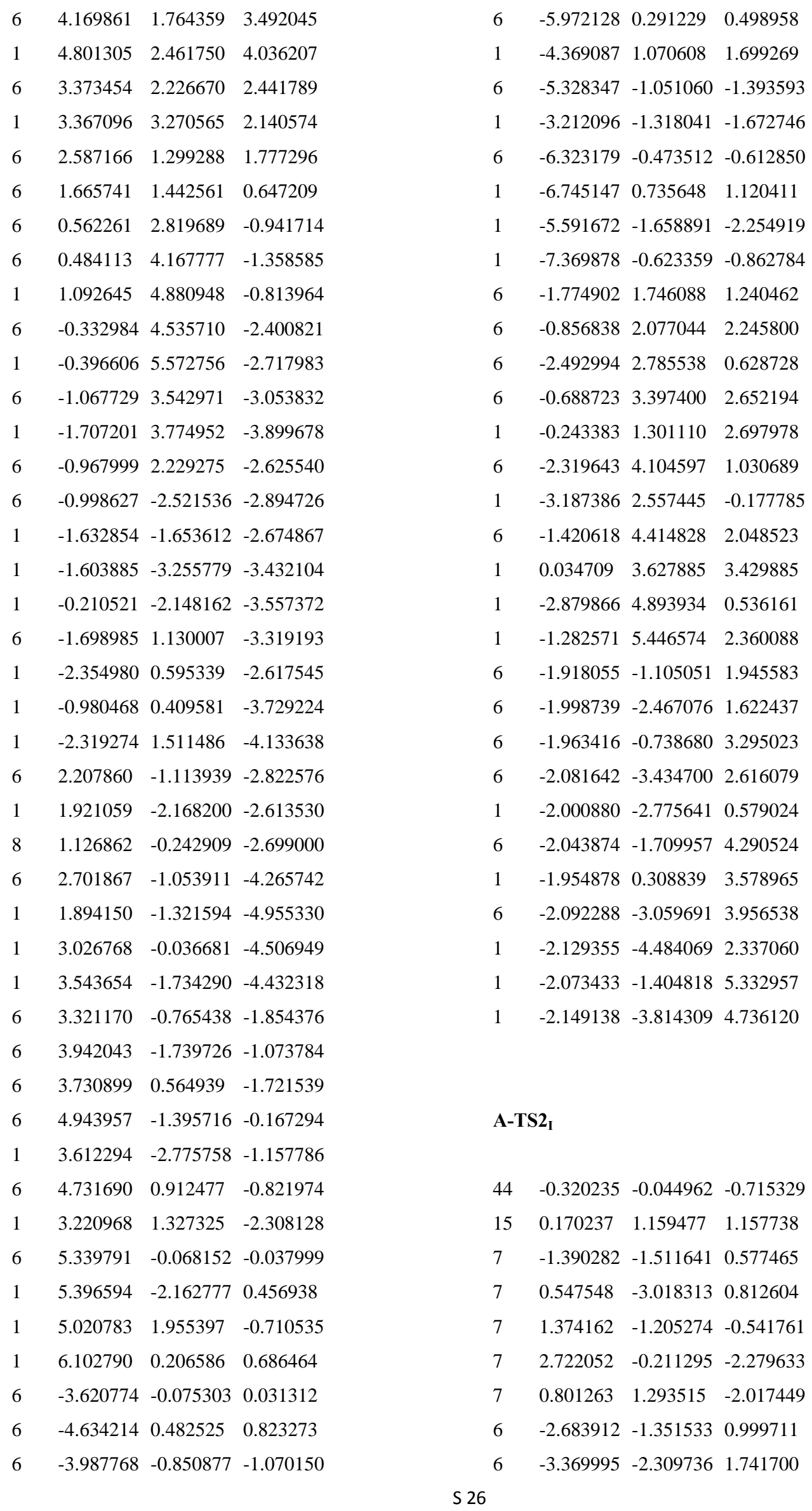


$1 \quad-2.450039-2.830104-1.689509$

$6 \quad-5.752865-1.997525-1.785139$

$1 \quad-6.511428 \quad-0.084049 \quad-2.426422$

$1 \quad-4.704546-3.791327-1.214978$

$1 \quad-6.737816 \quad-2.410089-1.582965$

$\begin{array}{lllll}6 & -1.768961 & 0.582218 & -3.761098\end{array}$

$1 \quad-2.209531 \quad 0.092116 \quad-4.636344$

$1 \quad-2.322660 \quad 1.509369 \quad-3.592025$

$\begin{array}{llll}1 & -0.731354 & 0.816062 & -3.992573\end{array}$

$\begin{array}{llll}6 & 0.788407 & 0.044784 & 2.491250\end{array}$

$\begin{array}{lllll}6 & -0.134650 & -0.586960 & 3.337254\end{array}$

$\begin{array}{lllll}6 & 2.137074 & -0.314314 & 2.592674\end{array}$

$\begin{array}{lllll}6 & 0.278926 & -1.549122 & 4.251949\end{array}$

$\begin{array}{lllll}1 & -1.189386 & -0.323169 & 3.279039\end{array}$

$\begin{array}{lllll}6 & 2.549896 & -1.281827 & 3.504708\end{array}$

$\begin{array}{llll}1 & 2.876164 & 0.157149 & 1.950932\end{array}$

$\begin{array}{lllll}6 & 1.622761 & -1.905042 & 4.334044\end{array}$

$1 \quad-0.456522-2.0304534 .891752$

$1 \quad 3.600900 \quad-1.5534963 .556488$

$\begin{array}{lllll}1 & 1.945291 & -2.664095 & 5.041430\end{array}$

$\begin{array}{llll}6 & -1.053012 & 2.171785 & 2.104063\end{array}$

$\begin{array}{llll}6 & -0.821680 & 2.489535 & 3.450949\end{array}$

$\begin{array}{llll}6 & -2.169502 & 2.729127 & 1.475108\end{array}$

$\begin{array}{llll}6 & -1.688100 & 3.326554 & 4.145435\end{array}$

$\begin{array}{llll}1 & 0.050314 & 2.080843 & 3.955975\end{array}$

$\begin{array}{llll}6 & -3.036356 & 3.572424 & 2.166537\end{array}$

$\begin{array}{lllll}1 & -2.367224 & 2.486300 & 0.434709\end{array}$

$\begin{array}{llll}6 & -2.799130 & 3.871125 & 3.504566\end{array}$

$1 \quad-1.492306 \quad 3.557435 \quad 5.189088$

$\begin{array}{llll}1 & -3.900684 & 3.991328 & 1.657894\end{array}$

$\begin{array}{llll}1 & -3.476254 & 4.525056 & 4.047464\end{array}$

$\begin{array}{llll}6 & 1.521701 & 2.406088 & 0.947646\end{array}$

$\begin{array}{llll}6 & 1.387700 & 3.750835 & 1.317304\end{array}$

$\begin{array}{llll}6 & 2.694688 & 2.017934 & 0.285669\end{array}$

$\begin{array}{lllll}6 & 2.397486 & 4.669509 & 1.038831\end{array}$

$\begin{array}{llll}1 & 0.485319 & 4.093998 & 1.815906\end{array}$

$\begin{array}{lllll}6 & 3.704744 & 2.932430 & 0.012098\end{array}$

$\begin{array}{lllll}1 & 2.808227 & 0.989192 & -0.041632\end{array}$

$\begin{array}{llll}6 & 3.558334 & 4.265499 & 0.387202\end{array}$

$\begin{array}{llll}1 & 2.270096 & 5.708341 & 1.332379\end{array}$

$1 \quad 4.598245 \quad 2.600629 \quad-0.510461$

$\begin{array}{llll}1 & 4.341367 & 4.986076 & 0.167471\end{array}$ 


$\begin{array}{llll}\mathbf{A}-5 & & & \\ & & & \\ 44 & 0.067350 & -0.495450 & -0.615759 \\ 15 & -2.031293 & -0.309927 & 0.174131 \\ 7 & 0.727415 & -1.880953 & 0.899646 \\ 7 & 2.002407 & -0.199057 & 2.189448 \\ 7 & 0.842371 & 1.084290 & 0.528399 \\ 7 & 0.135335 & 2.968537 & -0.776568 \\ 7 & -0.288081 & 0.983335 & -2.174494 \\ 6 & 0.364314 & -3.205484 & 0.914967 \\ 6 & 0.997039 & -4.145895 & 1.718848 \\ 1 & 0.662118 & -5.177035 & 1.670730 \\ 6 & 2.042608 & -3.768252 & 2.558824 \\ 1 & 2.564096 & -4.498722 & 3.171201 \\ 6 & 2.358321 & -2.434401 & 2.620966 \\ 1 & 3.108604 & -2.043537 & 3.298555 \\ 6 & -0.564269 & 0.606057 & -3.463433 \\ 6 & -0.730949 & -3.706625 & 0.031423 \\ 6 & 1.667633 & -1.476158 & 1.840405 \\ 6 & -1.618543 & -3.073637 & 0.077823 \\ 6 & 1.587692 & 0.918316 & 1.649229 \\ 6 & 1.920695 & 2.243186 & 2.197769 \\ 6 & -1.008740 & -4.719580 & 0.335582 \\ 6 & 2.630493 & 2.644312 & 3.319294 \\ 1 & 3.074845 & 1.909624 & 3.985030 \\ 6 & 2.747777 & 4.016114 & 3.557669 \\ 1 & 3.294270 & 4.365024 & 4.430010 \\ 6 & -0.432089 & -3.725393 & -1.020619 \\ 6 & -0.300219 & -0.796216 & -3.910702\end{array}$

$\begin{array}{llll}-1.082761 & -1.489040 & -3.591494\end{array}$

$1 \quad 0.634265 \quad-1.173927 \quad-3.491686$

$1 \quad-0.246665 \quad-0.822259-5.003017$

$6 \quad 5.431859-2.060889-1.678629$

$6 \quad 6.770336-1.691188-1.728770$

$6 \quad 7.126516 \quad-0.349468 \quad-1.614154$

$\begin{array}{lllll}6 & 6.141350 & 0.623874 & -1.441030\end{array}$

$\begin{array}{llll}6 & 4.805285 & 0.258403 & -1.386317\end{array}$

$6 \quad 4.433996-1.090027-1.511745$

$1 \quad 5.161610 \quad-3.110133 \quad-1.762132$

$1 \quad 7.537034 \quad-2.449526 \quad-1.857275$

$1 \quad 8.173277 \quad-0.061211-1.654381$

$1 \quad 6.421006 \quad 1.669021 \quad-1.344697$

$1 \quad 4.021270 \quad 0.996552 \quad-1.242444$

$\begin{array}{lllll}6 & 3.005699 & -1.444229 & -1.436558\end{array}$

$6 \quad 2.568464-2.870143-1.534795$

$1 \quad 3.081553 \quad-3.398596-2.343291$

$1 \quad 2.789245 \quad-3.404298 \quad-0.602630$

$1 \quad 1.485852 \quad-2.885737-1.690472$

$8 \quad 2.177891 \quad-0.532528-1.280517$

$1 \quad-0.417322-1.716701-1.562183$

$6 \quad-3.470382-0.955816-0.781651$

$6 \quad-4.776729-0.557502-0.459153$

$6 \quad-3.289445-1.894284-1.799791$

$6 \quad-5.867393 \quad-1.080540-1.144044$

$\begin{array}{llll}1 & -4.934901 & 0.167685 & 0.336415\end{array}$

$6 \quad-4.382174-2.418189-2.488297$

$1 \quad-2.274174 \quad-2.205080-2.037935$

$6 \quad-5.672132-2.011810-2.163240$

$\begin{array}{lllll}1 & -6.872827 & -0.761590 & -0.882077\end{array}$

$1 \quad-4.222779-3.144782-3.280865$

$\begin{array}{lllll}1 & -6.525101 & -2.417909 & -2.700742\end{array}$

$\begin{array}{llll}6 & -2.575984 & 1.430818 & 0.448519\end{array}$

$\begin{array}{llll}6 & -2.320009 & 2.113310 & 1.643658\end{array}$

$\begin{array}{lllll}6 & -3.134633 & 2.144457 & -0.620359\end{array}$

$\begin{array}{llll}6 & -2.614610 & 3.467875 & 1.765717\end{array}$

$1 \quad-1.884609 \quad 1.588197 \quad 2.489609$

$\begin{array}{llll}6 & -3.432878 & 3.497052 & -0.496073\end{array}$

$\begin{array}{lllll}1 & -3.331637 & 1.636248 & -1.563102\end{array}$

$\begin{array}{llll}6 & -3.172074 & 4.164477 & 0.697102\end{array}$

$\begin{array}{llll}1 & -2.407068 & 3.979081 & 2.702276\end{array}$

$1 \quad-3.861540 \quad 4.030869-1.340801$

$\begin{array}{llll}1 & -3.400536 & 5.222485 & 0.793608\end{array}$ 


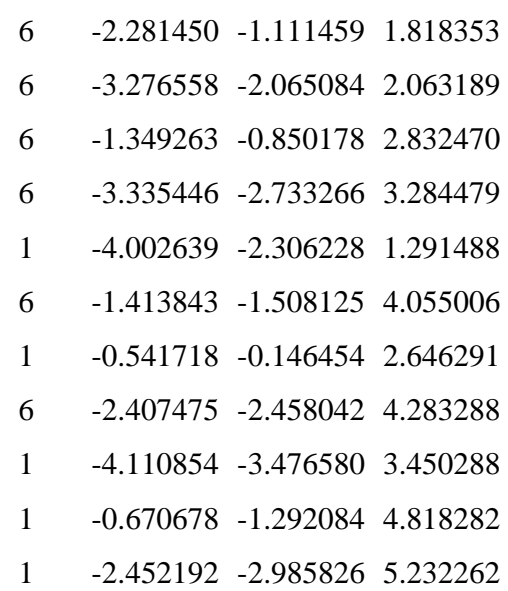

\section{A-rot-TS1 I}

$44 \quad 0.133968 \quad-0.165250 \quad-0.772916$

$1 \quad-0.248585-1.306418-1.834652$

$\begin{array}{lllll}15 & -1.851400 & -0.413308 & 0.292932\end{array}$

$\begin{array}{lllll}7 & 1.016184 & -1.550022 & 0.669882\end{array}$

$\begin{array}{llll}7 & 1.658353 & 0.133868 & 2.371940\end{array}$

$\begin{array}{llll}7 & 0.932608 & 1.374163 & 0.465455\end{array}$

$\begin{array}{lllll}7 & 1.276585 & 2.725547 & -1.429174\end{array}$

$\begin{array}{llll}7 & -0.613470 & 1.383375 & -2.120346\end{array}$

$\begin{array}{lllll}6 & 1.096468 & -2.893144 & 0.406096\end{array}$

$\begin{array}{lllll}6 & 1.445037 & -3.831042 & 1.377807\end{array}$

$\begin{array}{lllll}1 & 1.473115 & -4.876648 & 1.087842\end{array}$

$\begin{array}{lllll}6 & 1.768290 & -3.437191 & 2.668969\end{array}$

$\begin{array}{lllll}1 & 2.031739 & -4.162275 & 3.433537\end{array}$

$\begin{array}{lllll}6 & 1.783620 & -2.086990 & 2.926471\end{array}$

$\begin{array}{lllll}1 & 2.072249 & -1.677033 & 3.887329\end{array}$

$\begin{array}{lllll}6 & 1.431653 & -1.150011 & 1.933109\end{array}$

$\begin{array}{lllll}6 & 1.497254 & 1.237390 & 1.722458\end{array}$

$\begin{array}{llll}6 & 2.159266 & 2.500412 & 2.073178\end{array}$

$\begin{array}{llll}6 & 2.815290 & 2.942846 & 3.208697\end{array}$

$\begin{array}{llll}1 & 2.845439 & 2.331159 & 4.105724\end{array}$

$\begin{array}{llll}6 & 3.433914 & 4.196043 & 3.157079\end{array}$

$\begin{array}{llll}1 & 3.947953 & 4.576446 & 4.035549\end{array}$

$\begin{array}{llll}6 & 3.414735 & 4.965798 & 1.990092\end{array}$

$\begin{array}{llll}1 & 3.919464 & 5.927926 & 1.979297\end{array}$

$\begin{array}{llll}6 & 2.767218 & 4.513484 & 0.838271\end{array}$

$\begin{array}{lllll}1 & 2.763001 & 5.095296 & -0.079282\end{array}$

$\begin{array}{llll}6 & 2.132337 & 3.279694 & 0.898532\end{array}$

$\begin{array}{llll}6 & 1.362704 & 2.510163 & -0.065022\end{array}$
$6 \quad 0.003106 \quad 2.598304 \quad-2.040971$

$\begin{array}{lllll}6 & -0.515408 & 3.743486 & -2.638508\end{array}$

$\begin{array}{llll}1 & 0.039018 & 4.667537 & -2.520085\end{array}$

$\begin{array}{llll}6 & -1.685993 & 3.662935 & -3.374546\end{array}$

$1 \quad-2.117615 \quad 4.545551 \quad-3.838137$

$\begin{array}{llll}6 & -2.265190 & 2.414505 & -3.537335\end{array}$

$1 \quad-3.156202 \quad 2.283785 \quad-4.143626$

$6 \quad-1.710605 \quad 1.291676 \quad-2.924452$

$6 \quad 0.818929 \quad-3.415315 \quad-0.961306$

$1 \quad-0.255830-3.464436-1.155880$

$1 \quad 1.233485 \quad-4.422558-1.056969$

$1 \quad 1.249301 \quad-2.771475 \quad-1.728074$

$6 \quad-2.327366-0.039406-3.173815$

$1 \quad-1.554182-0.794837-3.329771$

$1 \quad-2.987877 \quad 0.010255 \quad-4.043262$

$1 \quad-2.931407 \quad-0.375394 \quad-2.325362$

$1 \quad 1.760315 \quad 1.742730 \quad-1.773883$

$6 \quad 3.621902-1.403557-1.596072$

$\begin{array}{lllll}6 & 4.146232 & -2.249727 & -0.618977\end{array}$

$6 \quad 4.516765 \quad-3.556277 \quad-0.931711$

$6 \quad 4.376697 \quad-4.027286-2.234482$

$6 \quad 3.861225-3.185570 \quad-3.220062$

$\begin{array}{lllll}6 & 3.483878 & -1.885420 & -2.901119\end{array}$

$1 \quad 4.242946 \quad-1.884072 \quad 0.403826$

$\begin{array}{lllll}1 & 4.907184 & -4.209055 & -0.153961\end{array}$

$14.662683 \quad-5.046738-2.480683$

$1 \quad 3.745592 \quad-3.549852-4.238158$

$1 \quad 3.046712 \quad-1.227876 \quad-3.648799$

$\begin{array}{lllll}6 & 3.215335 & 0.019535 & -1.275687\end{array}$

$1 \quad 3.146883 \quad 0.099975 \quad-0.169376$

$\begin{array}{lllll}6 & 4.290397 & 0.999427 & -1.744509\end{array}$

$1 \quad 4.386630 \quad 0.953554 \quad-2.834387$

$1 \quad 4.033325 \quad 2.028588 \quad-1.470158$

$1 \quad 5.264488 \quad 0.762677 \quad-1.304325$

$8 \quad 1.988430 \quad 0.321272 \quad-1.886144$

$6 \quad-1.723490 \quad-0.530600 \quad 2.133051$

$\begin{array}{lllll}6 & -1.577237 & -1.788433 & 2.733859\end{array}$

$\begin{array}{lllll}6 & -1.645478 & 0.606339 & 2.945003\end{array}$

$\begin{array}{lllll}6 & -1.375093 & -1.904732 & 4.104759\end{array}$

$1 \quad-1.617884-2.6860592 .119763$

$\begin{array}{lllll}6 & -1.439990 & 0.490325 & 4.316640\end{array}$

$1 \quad-1.749645 \quad 1.594994 \quad 2.505055$

$\begin{array}{llll}6 & -1.305890 & -0.765672 & 4.902092\end{array}$ 


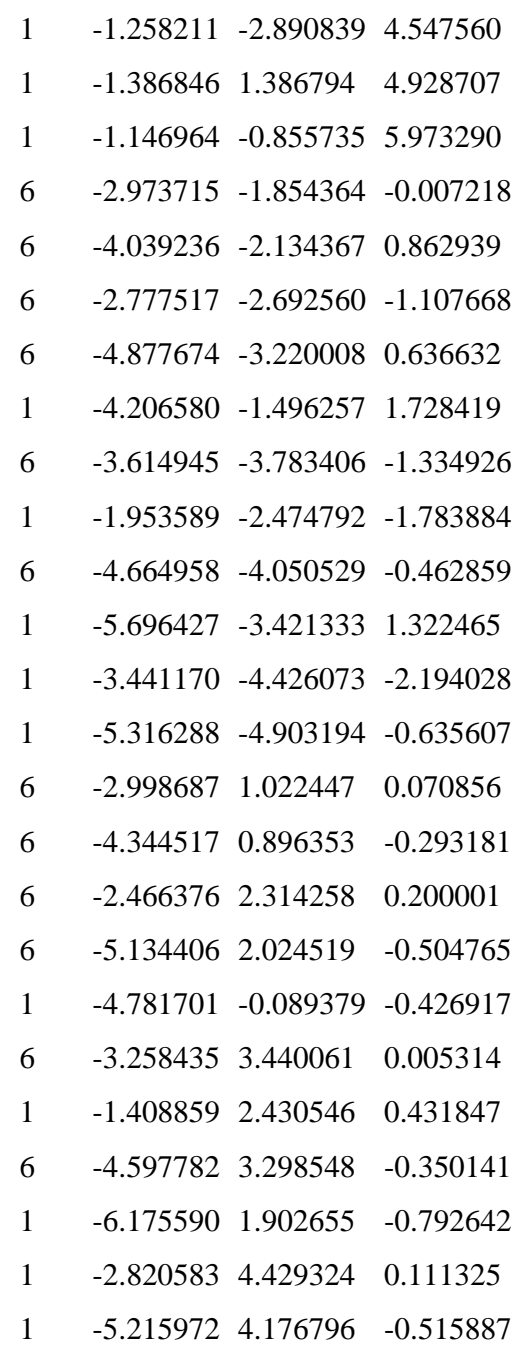

\section{A-rot-TS1 1 -PS}

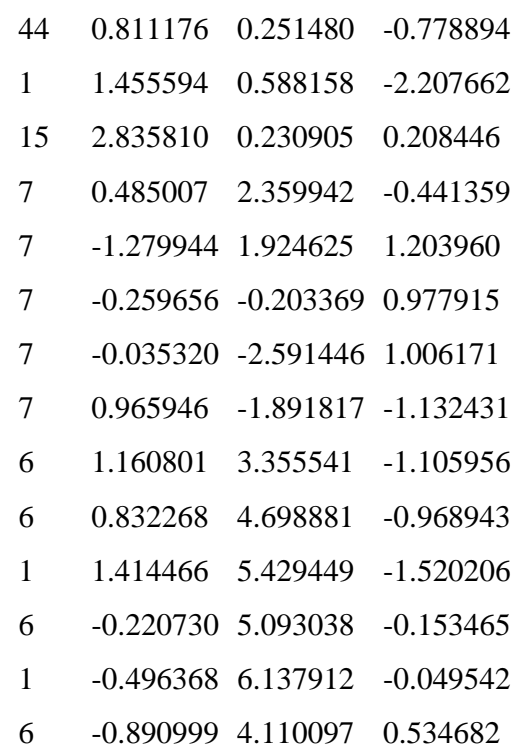

$\begin{array}{llll}1 & -1.706801 & 4.337448 & 1.212598\end{array}$

$\begin{array}{lllll}6 & -0.508517 & 2.768107 & 0.392893\end{array}$

$\begin{array}{lllll}6 & -1.152408 & 0.615729 & 1.502067\end{array}$

$\begin{array}{llll}6 & -2.027703 & -0.073082 & 2.446712\end{array}$

$\begin{array}{lllll}6 & -3.103558 & 0.329439 & 3.226799\end{array}$

$1 \quad-3.464618 \quad 1.355076 \quad 3.211377$

$\begin{array}{lllll}6 & -3.719045 & -0.635188 & 4.028599\end{array}$

$1 \quad-4.568838-0.355320 \quad 4.644584$

$\begin{array}{lllll}6 & -3.258967 & -1.954843 & 4.050457\end{array}$

$1 \quad-3.757921 \quad-2.680500 \quad 4.686848$

$\begin{array}{lllll}6 & -2.179755 & -2.356789 & 3.258465\end{array}$

$1 \quad-1.831169 \quad-3.385526 \quad 3.245799$

$\begin{array}{llll}6 & -1.573312 & -1.401333 & 2.461914\end{array}$

$6 \quad-0.514693 \quad-1.4783351 .447764$

$\begin{array}{lllll}6 & 0.678005 & -2.814193 & -0.141321\end{array}$

$\begin{array}{lllll}6 & 1.023727 & -4.175748 & -0.275009\end{array}$

$\begin{array}{llll}1 & 0.777567 & -4.813735 & 0.565902\end{array}$

$\begin{array}{lllll}6 & 1.603263 & -4.650504 & -1.424897\end{array}$

$1 \quad 1.871651-5.697260-1.534265$

$\begin{array}{lllll}6 & 1.777957 & -3.746264 & -2.466944\end{array}$

$\begin{array}{llll}1 & 2.169175 & -4.069802 & -3.426457\end{array}$

$\begin{array}{lllll}6 & 1.448369 & -2.402084 & -2.312499\end{array}$

$\begin{array}{lllll}6 & 2.287964 & 3.020383 & -2.018408\end{array}$

$\begin{array}{lllll}1 & 3.058893 & 2.439958 & -1.506627\end{array}$

$\begin{array}{llll}1 & 2.743638 & 3.940352 & -2.393038\end{array}$

$\begin{array}{lllll}1 & 1.949637 & 2.411474 & -2.859664\end{array}$

$\begin{array}{lllll}6 & 1.648455 & -1.531669 & -3.511407\end{array}$

$1 \quad 2.593512 \quad-0.984897 \quad-3.453009$

$1 \quad 0.867262 \quad-0.780121-3.615575$

$1 \quad 1.680203 \quad-2.157556 \quad-4.407732$

$6 \quad-1.820376-0.653097-2.404162$

$\begin{array}{lllll}8 & -1.313678 & 0.414294 & -1.626428\end{array}$

$\begin{array}{llll}1 & -2.280981 & 2.136208 & 0.992210\end{array}$

$1 \quad-0.989808-1.032648-3.018400$

$\begin{array}{lllll}8 & -3.047901 & 1.770257 & -0.542532\end{array}$

$1 \quad-2.160693 \quad 1.054619 \quad-1.172041$

$6 \quad-2.339613 \quad-1.812547 \quad-1.579234$

$\begin{array}{lllll}6 & -2.031719 & -3.131057 & -1.917847\end{array}$

$6 \quad-3.206872-1.576801-0.509410$

$6 \quad-2.566464 \quad-4.195396 \quad-1.196107$

$1 \quad-1.349743 \quad-3.321420-2.748129$

$6 \quad-3.751360-2.6371180 .207216$

$1 \quad-3.446326 \quad-0.547156 \quad-0.247746$ 


\begin{tabular}{llll}
6 & 3.826709 & 0.830105 & 1.786048 \\
1 & 4.119838 & 0.947446 & 2.837464 \\
1 & 4.627559 & 0.271306 & 1.290673 \\
1 & 3.778230 & 1.830905 & 1.342397 \\
6 & 1.451422 & 0.859189 & 2.542757 \\
1 & 1.272557 & 1.871803 & 2.169346 \\
1 & 0.486761 & 0.329793 & 2.558009 \\
1 & 1.799816 & 0.926420 & 3.580757 \\
6 & 2.972659 & -0.560728 & -1.321978 \\
6 & 3.609395 & -1.859621 & -0.835155 \\
1 & 4.338884 & -1.686876 & -0.038069 \\
1 & 2.864931 & -2.577623 & -0.475349 \\
1 & 4.147679 & -2.332575 & -1.665636 \\
6 & 4.064362 & 0.455061 & -1.667264 \\
1 & 4.751309 & 0.010213 & -2.397731 \\
1 & 3.639999 & 1.356203 & -2.118248 \\
1 & 4.659604 & 0.755736 & -0.800570 \\
6 & 2.169321 & -0.849081 & -2.593471 \\
1 & 1.457985 & -1.665870 & -2.442269 \\
1 & 1.608832 & 0.033620 & -2.919941 \\
1 & 2.856598 & -1.135630 & -3.399247 \\
6 & -3.272129 & -0.524007 & -1.275602 \\
1 & -2.723471 & 0.229441 & -1.850272 \\
1 & -4.327607 & -0.203845 & -1.256667 \\
6 & -3.146944 & -1.882372 & -1.923887 \\
1 & -3.799199 & -2.627646 & -1.458006 \\
1 & -3.432181 & -1.814925 & -2.976448 \\
1 & -2.116746 & -2.246425 & -1.873180 \\
6 & -3.300964 & -1.502901 & 0.993813 \\
1 & -2.966600 & -2.472443 & 0.614036 \\
1 & -2.815150 & -1.375289 & 1.968723 \\
6 & -4.811123 & -1.479664 & 1.147120 \\
1 & -5.125165 & -2.266123 & 1.837747 \\
1 & -5.173601 & -0.528861 & 1.548938 \\
\hline & & & \\
\hline & -357185 & 2.511573 & -0.724583
\end{tabular}

$$
\begin{array}{llll}
\mathbf{B}^{-T} & \text { iso } \mathbf{- 1} \\
& & & \\
44 & 0.470956 & -0.603062 & -0.081224 \\
1 & 0.343050 & -0.130085 & 1.422684 \\
15 & -1.711767 & 0.102518 & 0.166256
\end{array}
$$

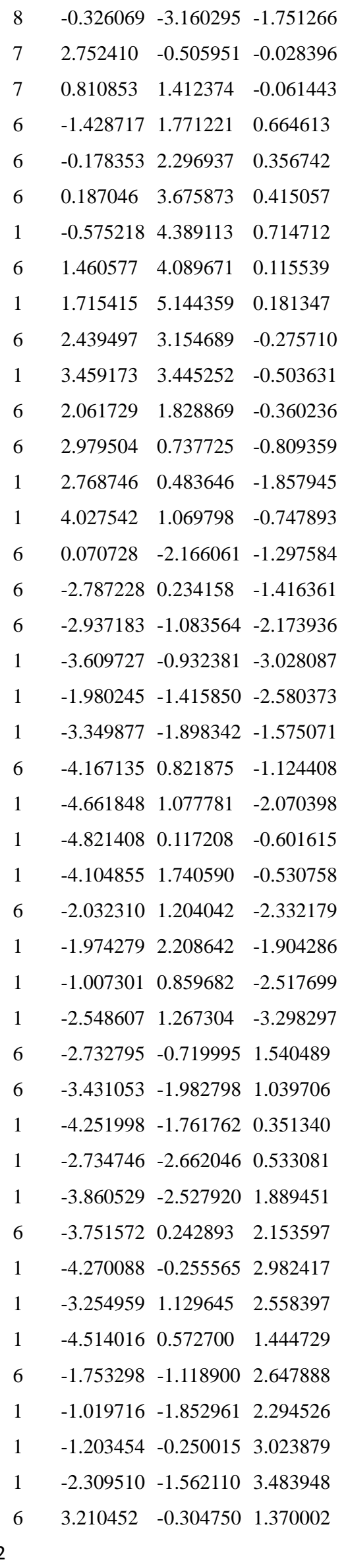




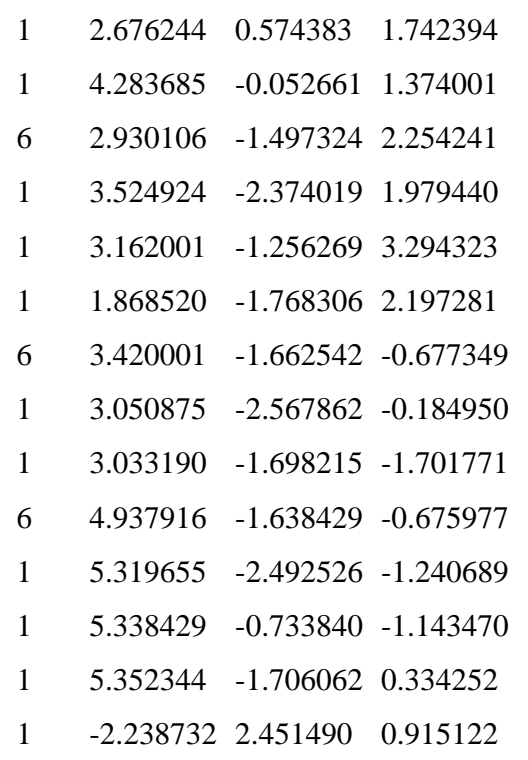

B $_{\text {isomer-1-a }}$

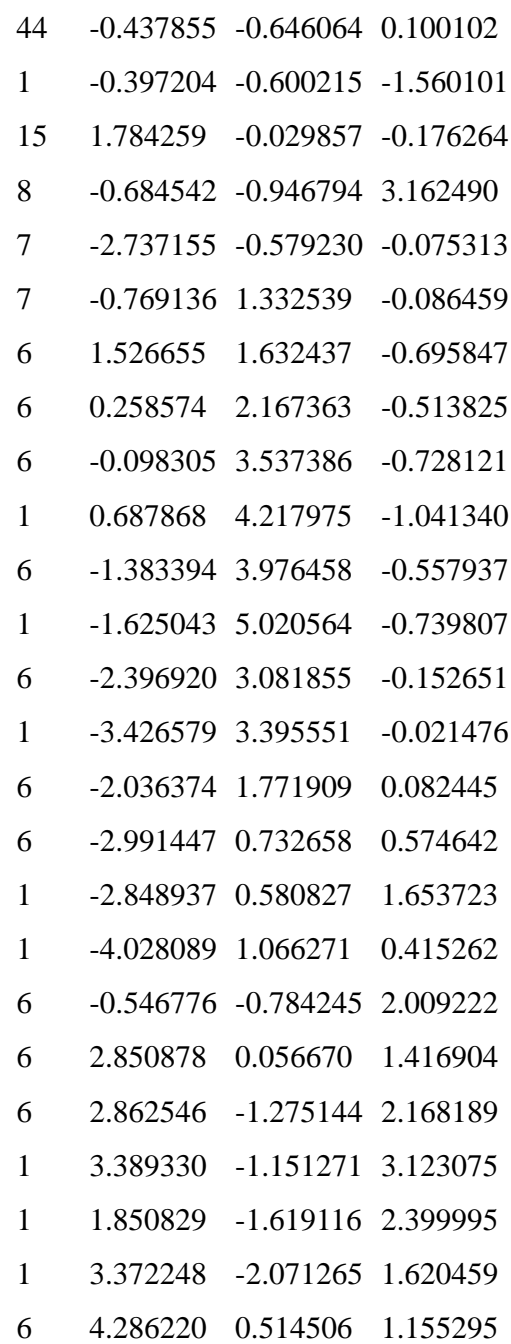

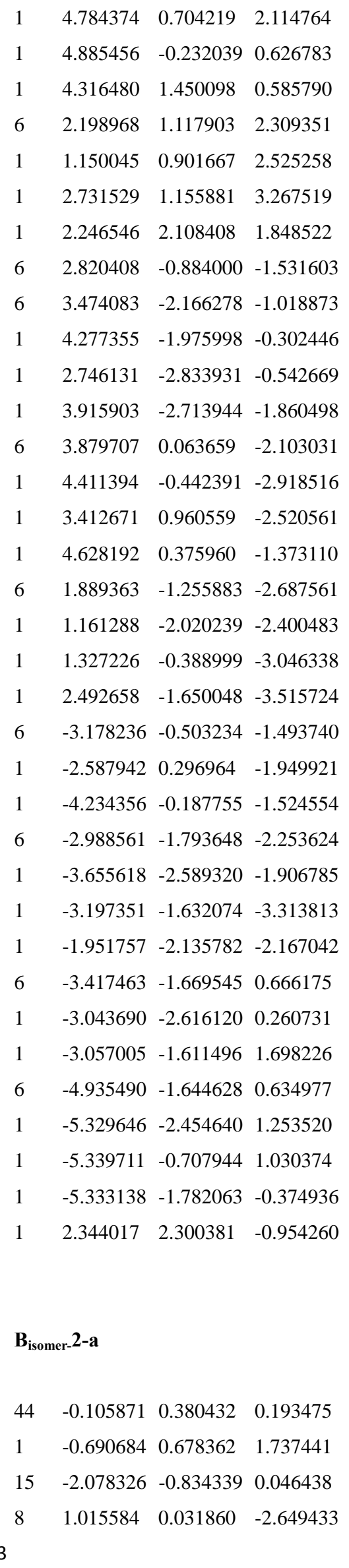




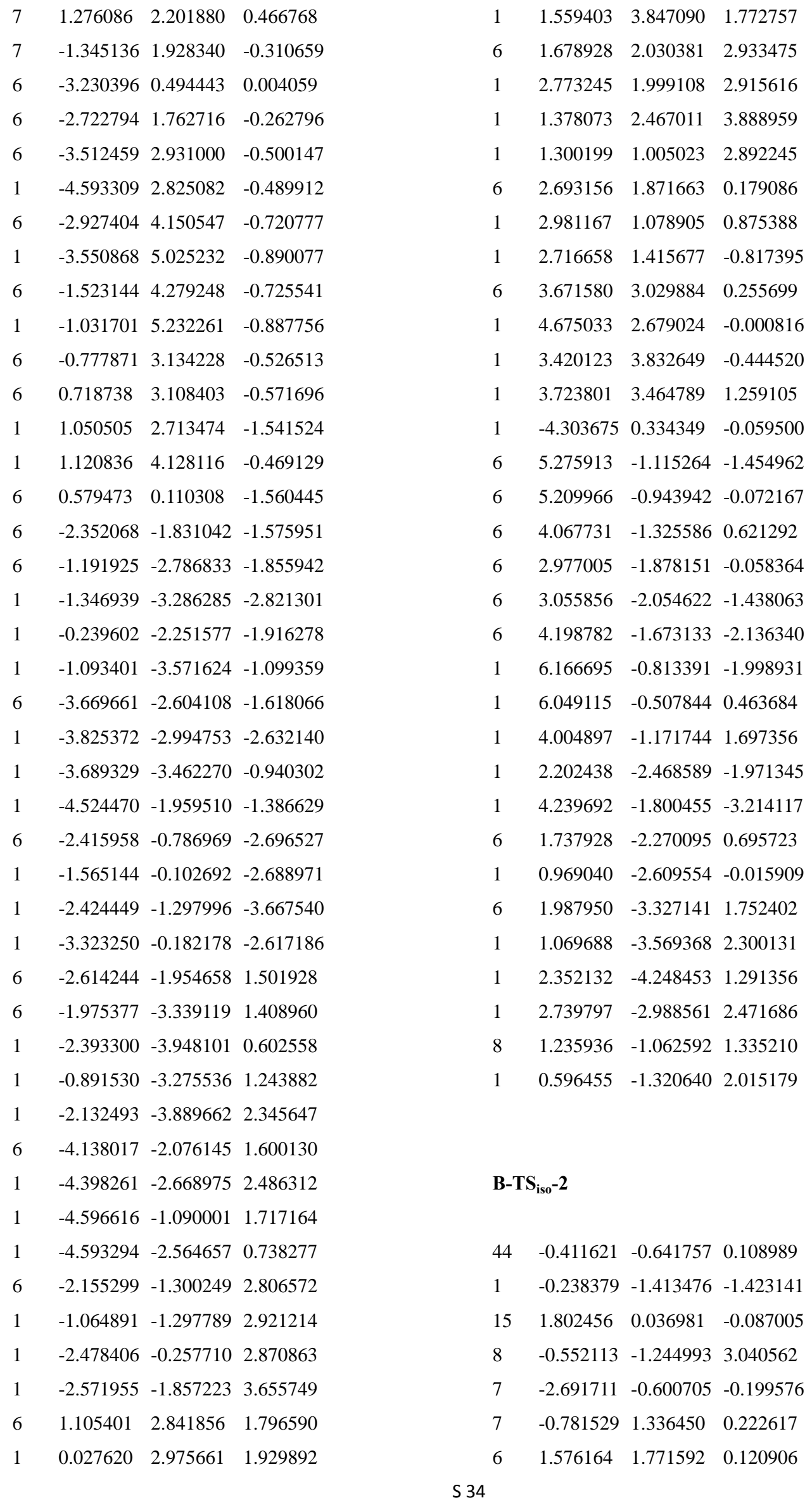




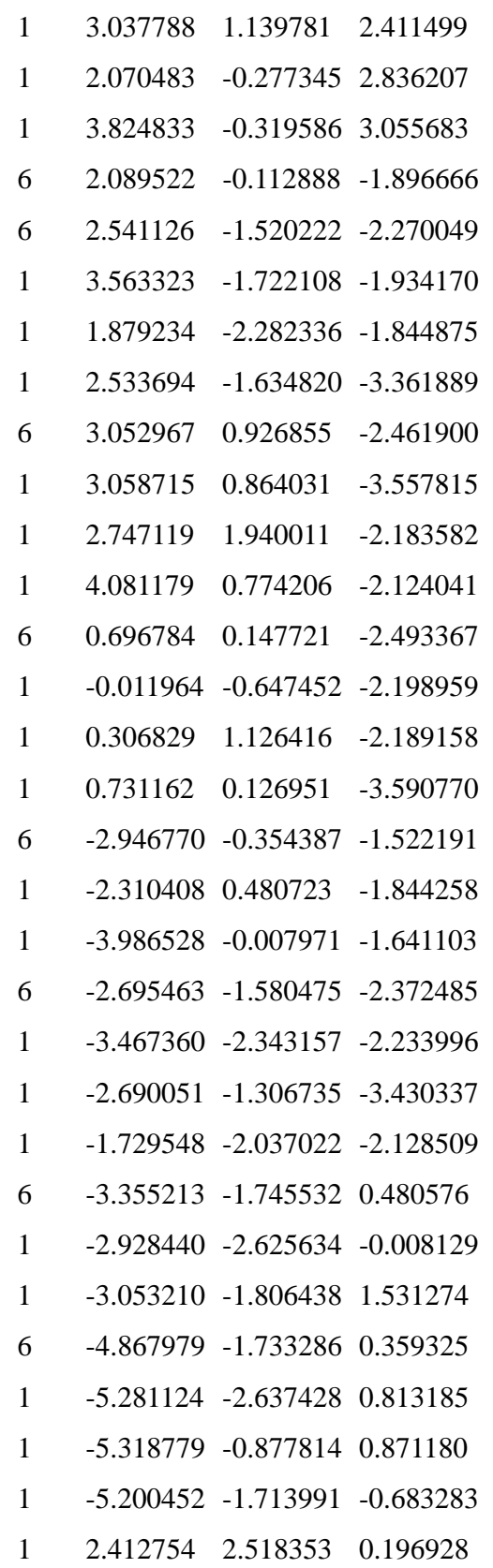

$$
\begin{array}{llll}
\mathbf{B}_{\text {isomer-2-b }} & & \\
& & & \\
44 & 0.158623 & -0.209214 & -0.501255 \\
15 & 2.369500 & 0.534750 & -0.091544 \\
7 & -1.714482 & -1.741295 & -0.291013 \\
7 & 0.600102 & -1.421006 & 1.243643 \\
6 & 2.814792 & -0.519913 & 1.250030 \\
6 & 1.884993 & -1.415441 & 1.769251 \\
6 & 2.158712 & -2.334634 & 2.834110 \\
1 & 3.159910 & -2.353271 & 3.255252
\end{array}
$$

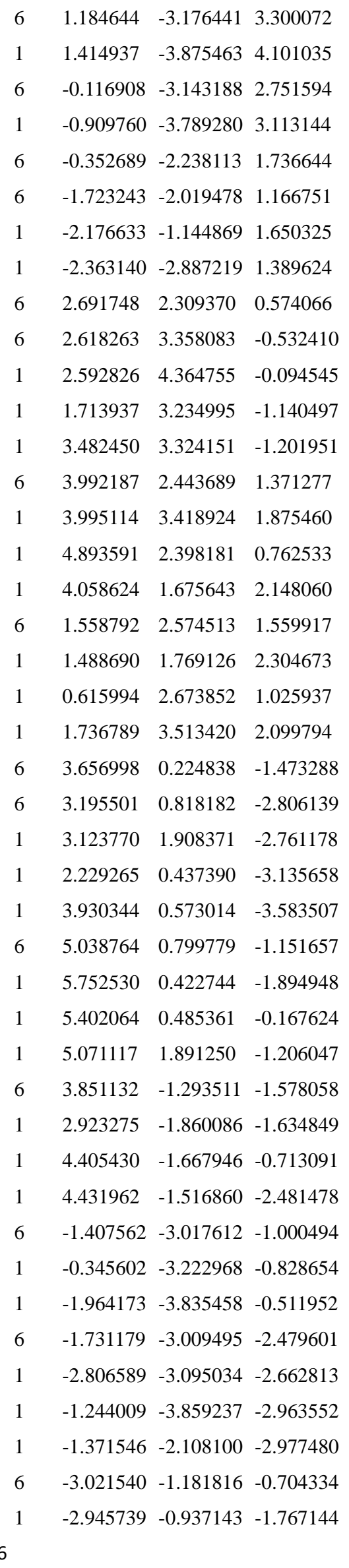

$\begin{array}{lllll}1 & 1.414937 & -3.875463 & 4.101035\end{array}$

$\begin{array}{llll}6 & -0.116908 & -3.143188 & 2.751594\end{array}$

$\begin{array}{llll}1 & -0.909760 & -3.789280 & 3.113144\end{array}$

$\begin{array}{lllll}6 & -0.352689 & -2.238113 & 1.736644\end{array}$

$6 \quad-1.723243 \quad-2.019478 \quad 1.166751$

$1 \quad-2.176633-1.1448691 .650325$

$1 \quad-2.363140 \quad-2.887219 \quad 1.389624$

$\begin{array}{lllll}6 & 2.691748 & 2.309370 & 0.574066\end{array}$

$\begin{array}{llll}6 & 2.618263 & 3.358083 & -0.532410\end{array}$

$\begin{array}{llll}2.592826 & 4.364755 & -0.094545\end{array}$

$\begin{array}{lll}1.713937 & 3.234995 & -1.140497\end{array}$

$\begin{array}{llll}3.482450 & 3.324151 & -1.201951\end{array}$

$\begin{array}{llll} & 3.995114 & 3.418924 \quad 1.875460\end{array}$

$\begin{array}{llll}1 & 4.893591 & 2.398181 & 0.762533\end{array}$

$\begin{array}{llll}1 & 4.058624 & 1.675643 & 2.148060\end{array}$

$\begin{array}{llll}1.558792 & 2.574513 & 1.559917\end{array}$

$\begin{array}{llll}1.488690 & 1.769126 & 2.304673\end{array}$

$\begin{array}{llll}1 & 0.615994 & 2.673852 & 1.025937\end{array}$

$1 \quad 1.736789 \quad 3.513420 \quad 2.099794$

$\begin{array}{llll}6 & 3.656998 & 0.224838 & -1.473288\end{array}$

$\begin{array}{llll}3.195501 & 0.818182 & -2.806139\end{array}$

$\begin{array}{llll}3.123770 & 1.908371 & -2.761178\end{array}$

$\begin{array}{llll}1 & 2.229265 & 0.437390 & -3.135658\end{array}$

$\begin{array}{llll}3.930344 & 0.573014 & -3.583507\end{array}$

$\begin{array}{llll}6 & 5.038764 & 0.799779 & -1.151657\end{array}$

$\begin{array}{lll}5.402064 & 0.485361 & -0.167624\end{array}$

$1 \quad 5.071117 \quad 1.891250 \quad-1.206047$

$\begin{array}{llll}3.851132 & -1.293511 & -1.578058\end{array}$

$\begin{array}{llll}2.923275 & -1.860086 & -1.634849\end{array}$

$\begin{array}{llll}4.405430 & -1.667946 & -0.713091\end{array}$

$\begin{array}{llll}4.431962 & -1.516860 & -2.481478\end{array}$

$\begin{array}{llll}6 & -1.407562 & -3.017612 & -1.000494\end{array}$

$1 \quad-0.345602-3.222968-0.828654$

$\begin{array}{llll}-1.964173 & -3.835458 & -0.511952\end{array}$

$6 \quad-1.731179-3.009495-2.479601$

$-2.806589-3.095034-2.662813$

$1 \quad-1.244009-3.859237-2.963552$

$1 \quad-1.371546-2.108100 \quad-2.977480$

$\begin{array}{llll}-3.021540 & -1.181816 & -0.704334\end{array}$ 


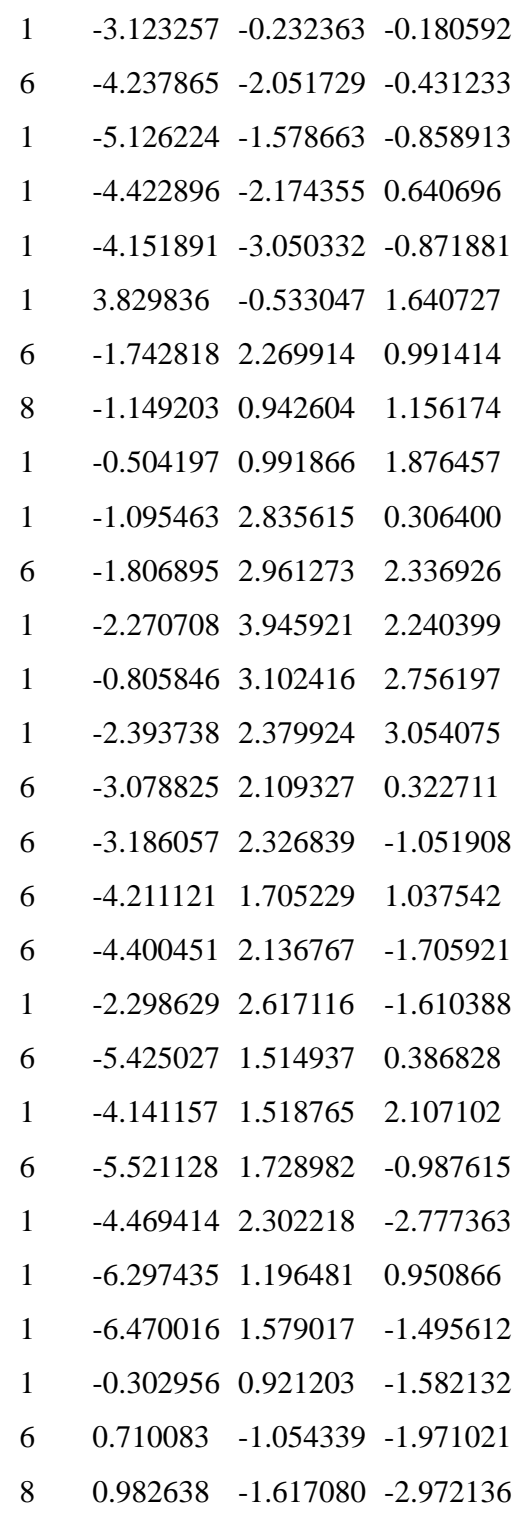

\section{B-TS1}

$\begin{array}{llll}44 & 0.329384 & 0.442841 & -0.638679 \\ 1 & 1.690713 & 0.645614 & -1.524159 \\ 15 & 1.138866 & -1.623439 & -0.174690 \\ 8 & -1.244746 & -0.288150 & -3.097624 \\ 7 & 0.308518 & 2.780007 & -0.675825 \\ 7 & 1.320193 & 0.948328 & 1.182981 \\ 6 & 1.464416 & -1.425020 & 1.642047 \\ 6 & 1.808052 & -0.012912 & 1.998900 \\ 6 & 2.525797 & 0.334910 & 3.141931 \\ 1 & 2.914861 & -0.446240 & 3.787343 \\ 6 & 2.722347 & 1.676966 & 3.443349\end{array}$

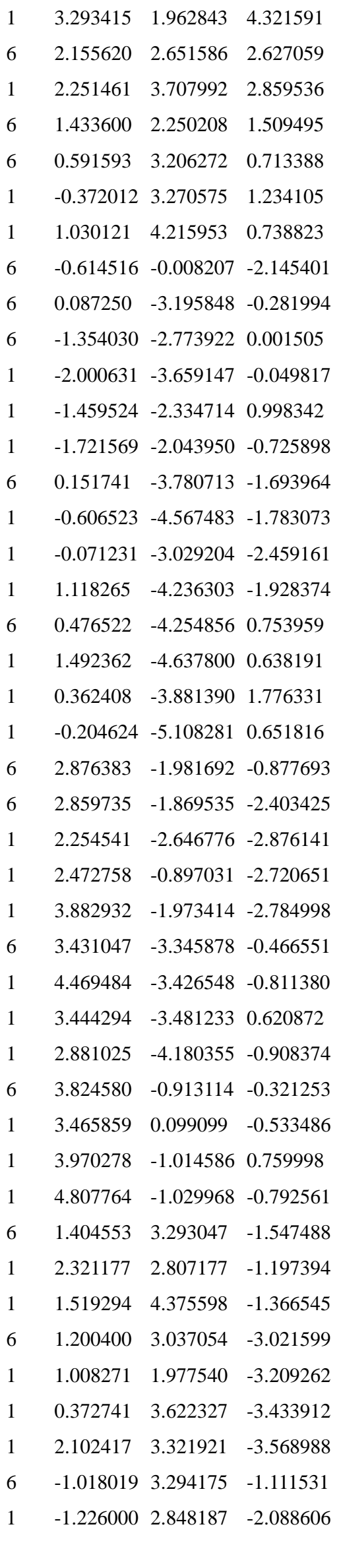




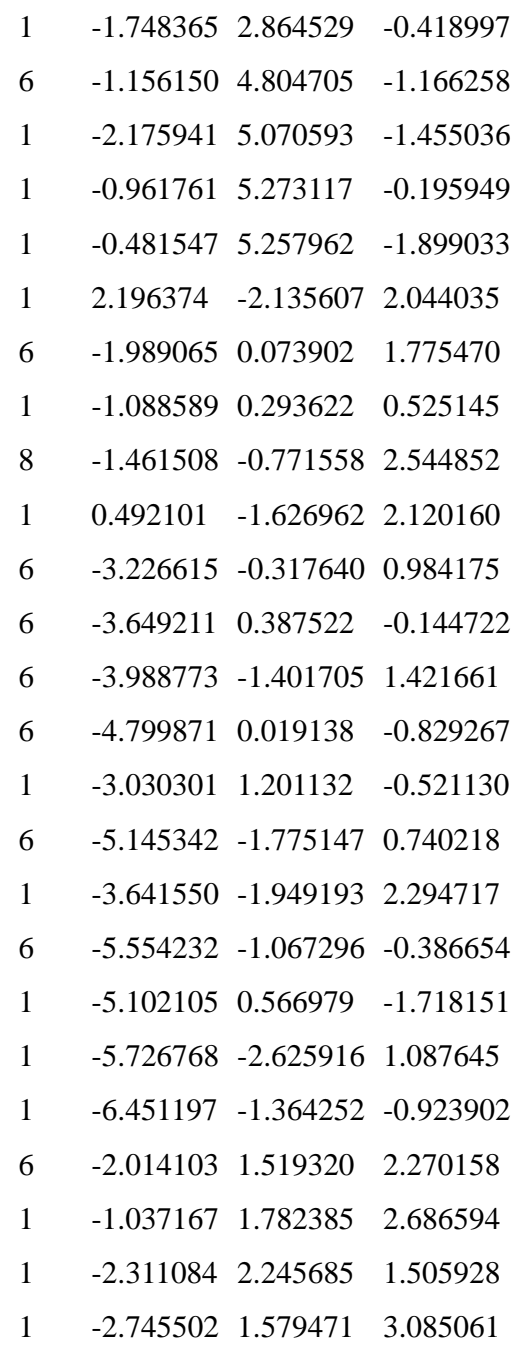

$$
\begin{array}{llll}
\text { B-TS1 } & \text { o-PS } & & \\
& & & \\
15 & 1.011814 & -2.178768 & -0.423760 \\
6 & 2.151476 & -3.506762 & 0.325652 \\
6 & 2.300434 & -3.194551 & 1.819929 \\
1 & 1.360748 & -3.327232 & 2.365915 \\
1 & 2.660085 & -2.175488 & 1.996278 \\
1 & 3.028841 & -3.887784 & 2.256458 \\
6 & 3.540926 & -3.458208 & -0.314248 \\
1 & 3.978926 & -2.458482 & -0.240820 \\
1 & 3.536169 & -3.747732 & -1.366692 \\
6 & 0.348034 & -2.753425 & -2.097614 \\
6 & -0.142972 & -1.506594 & -2.839337 \\
1 & 0.692838 & -0.864354 & -3.134364 \\
1 & -0.860667 & -0.905369 & -2.274974 \\
1 & -0.649976 & -1.826805 & -3.757506
\end{array}
$$

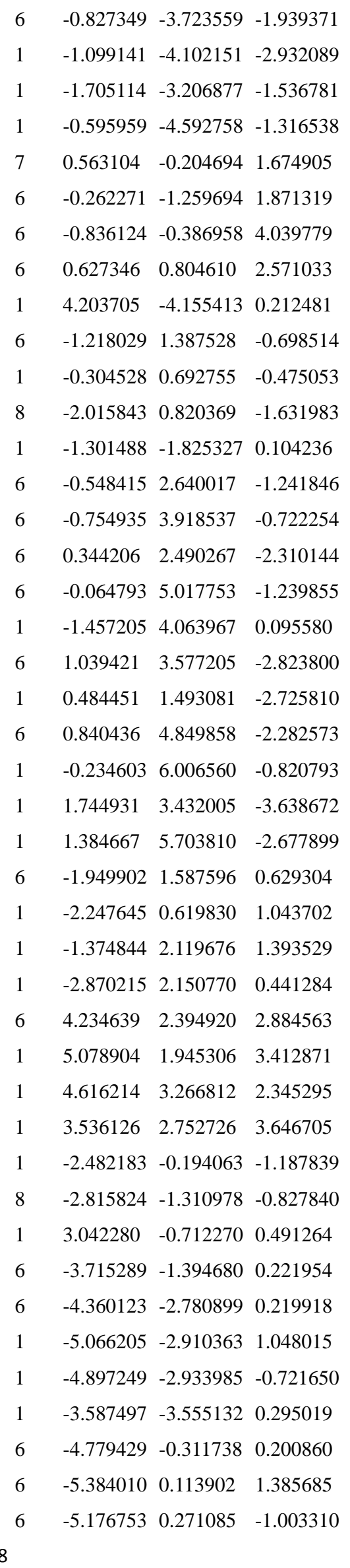

$\begin{array}{llll}-0.234603 & 6.006560 & -0.820793\end{array}$

$\begin{array}{llll}1.744931 & 3.432005 & -3.638672\end{array}$

$\begin{array}{llll}1.384667 & 5.703810 & -2.677899\end{array}$

$\begin{array}{llll}-1.949902 & 1.587596 & 0.629304\end{array}$

$\begin{array}{lll}-2.247645 & 0.619830 & 1.043702\end{array}$

$\begin{array}{lll}-1.374844 & 2.119676 & 1.393529\end{array}$

$\begin{array}{llll}5.078904 & 1.945306 & 3.412871\end{array}$

3.646705

$\begin{array}{llll}-2.482183 & -0.194063 & -1.187839\end{array}$

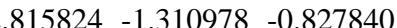

$\begin{array}{llll}3.042280 & -0.712270 & 0.491264\end{array}$

$\begin{array}{llll}-3.715289 & -1.394680 & 0.221954\end{array}$

$\begin{array}{llll}-4.360123 & -2.780899 & 0.219918\end{array}$

$-5.066205-2.9103631 .048015$

$\begin{array}{lll}-4.897249 & -2.933985 & -0.721650\end{array}$

$\begin{array}{llll}-3.587497 & -3.555132 & 0.295019\end{array}$

$\begin{array}{lll}-4.779429 & -0.311738 & 0.200860\end{array}$

$\begin{array}{lll}-5.384010 & 0.113902 & 1.385685\end{array}$ 


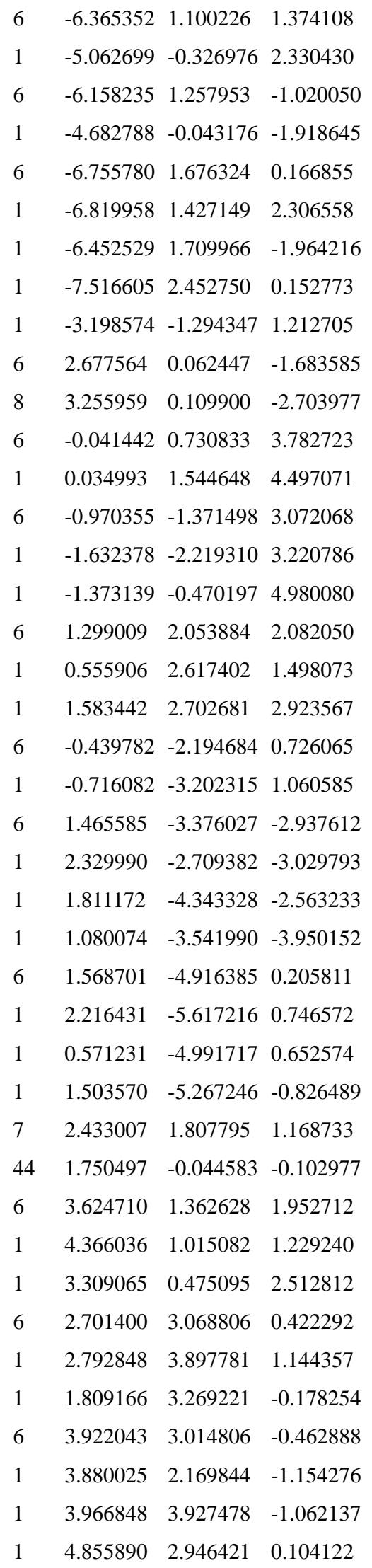

$$
\begin{aligned}
& \text { B-2 } \\
& 44 \quad-0.451518-0.6192020 .098010 \\
& 1 \quad-0.476796 \quad-0.794903-1.590587 \\
& 15 \quad \begin{array}{llll}
1.699697 & -0.003286 & -0.069925
\end{array} \\
& 8 \quad 0.070056 \quad-3.5491990 .558418 \\
& \begin{array}{lllll}
7 & -2.747113 & -0.410645 & 0.125935
\end{array} \\
& \begin{array}{lllll}
7 & -0.768239 & 1.457630 & -0.243851
\end{array} \\
& \begin{array}{lllll}
6 & 1.508729 & 1.592434 & -1.027452
\end{array} \\
& \begin{array}{lllll}
6 & 0.205004 & 2.262947 & -0.723132
\end{array} \\
& \begin{array}{llll}
6 & -0.041694 & 3.617551 & -0.927913
\end{array} \\
& 1 \quad 0.752824 \quad 4.256712-1.301513 \\
& \begin{array}{llll}
6 & -1.308278 & 4.130848 & -0.663425
\end{array} \\
& 1 \quad-1.517160 \quad 5.183625 \quad-0.828824 \\
& \begin{array}{lllll}
6 & -2.303707 & 3.282241 & -0.187433
\end{array} \\
& 1 \quad-3.301724 \quad 3.650516 \quad 0.031124 \\
& \begin{array}{lllll}
6 & -1.995147 & 1.944616 & 0.026958
\end{array} \\
& \begin{array}{lllll}
6 & -2.919623 & 0.957484 & 0.667600
\end{array} \\
& \begin{array}{llll}
1 & -2.651057 & 0.899113 & 1.732556
\end{array} \\
& 1 \quad-3.964344 \quad 1.297942 \quad 0.587264 \\
& \begin{array}{lllll}
6 & -0.145686 & -2.405387 & 0.383502
\end{array} \\
& \begin{array}{llll}
6 & 2.560397 & 0.559319 & 1.535366
\end{array} \\
& \begin{array}{lllll}
6 & 2.473173 & -0.573913 & 2.560100
\end{array} \\
& \begin{array}{llll}
1 & 2.867265 & -0.224137 & 3.522226
\end{array} \\
& \begin{array}{lllll}
1 & 1.432689 & -0.881590 & 2.703491
\end{array} \\
& \begin{array}{llll}
1 & 3.053141 & -1.453971 & 2.269060
\end{array} \\
& \begin{array}{llll}
6 & 4.008037 & 1.016781 & 1.373687
\end{array} \\
& \begin{array}{llll}
1 & 4.362071 & 1.429594 & 2.326755
\end{array} \\
& 14.686860 \quad 0.201601 \quad 1.107661 \\
& 1 \quad 4.112642 \quad 1.809207 \quad 0.623650 \\
& \begin{array}{llll}
6 & 1.744114 & 1.743060 & 2.067718
\end{array} \\
& 1 \quad 1.862165 \quad 2.640130 \quad 1.448863 \\
& \begin{array}{llll}
1 & 0.680214 & 1.490971 & 2.133902
\end{array} \\
& \begin{array}{llll}
1 & 2.096778 & 1.996427 & 3.074753
\end{array} \\
& 6 \quad 2.934182 \quad-0.971595 \quad-1.140925 \\
& 6 \quad 3.548684 \quad-2.100814 \quad-0.310924 \\
& 14.230135 \quad-1.7328310 .461253 \\
& \begin{array}{llll}
1 & 2.781784 & -2.715582 & 0.171906
\end{array} \\
& 1 \quad 4.128495-2.758322-0.969802 \\
& 6 \quad 4.039853 \quad-0.099471 \quad-1.744520 \\
& 14.689867 \quad-0.730700 \quad-2.362784 \\
& \begin{array}{llll}
1 & 3.635570 & 0.676377 & -2.402918
\end{array} \\
& \begin{array}{llll}
1 & 4.674600 & 0.384490 & -1.000767
\end{array}
\end{aligned}
$$




$\begin{array}{llll}6 & 2.156248 & -1.604480 & -2.298249 \\ 1 & 1.394785 & -2.302219 & -1.944079 \\ 1 & 1.639120 & -0.858119 & -2.910067 \\ 1 & 2.863759 & -2.142397 & -2.942201 \\ 6 & -3.351437 & -0.471799 & -1.230409 \\ 1 & -2.787480 & 0.238549 & -1.844432 \\ 1 & -4.392584 & -0.107897 & -1.180753 \\ 6 & -3.299496 & -1.846724 & -1.850815 \\ 1 & -3.962741 & -2.556345 & -1.346256 \\ 1 & -3.613456 & -1.791889 & -2.896114 \\ 1 & -2.275210 & -2.230552 & -1.820917 \\ 6 & -3.340026 & -1.405048 & 1.059767 \\ 1 & -3.050778 & -2.394664 & 0.696325 \\ 1 & -2.811334 & -1.267358 & 2.007772 \\ 6 & -4.844636 & -1.320247 & 1.239605 \\ 1 & -5.170913 & -2.071996 & 1.962690 \\ 1 & -5.165548 & -0.345394 & 1.620508 \\ 1 & -5.386426 & -1.510532 & 0.307413 \\ 1 & 1.494564 & 1.277693 & -2.080347 \\ 1 & 2.352441 & 2.284748 & -0.912521 \\ 1 & -0.618001 & -0.334228 & 1.785583\end{array}$

\section{B-TS2 0}

$\begin{array}{llll}44 & 0.449042 & -0.596309 & -0.139395 \\ 1 & 0.532029 & -0.705915 & 1.462742 \\ 15 & -1.742396 & 0.022583 & -0.076793 \\ 8 & 0.031382 & -3.577974 & -0.202920 \\ 7 & 2.780898 & -0.399679 & 0.029245 \\ 7 & 0.789840 & 1.498446 & -0.241051 \\ 6 & -1.443614 & 1.578624 & -0.979258 \\ 6 & -0.270676 & 2.300462 & -0.552209 \\ 6 & -0.078153 & 3.696072 & -0.605719 \\ 1 & -0.923635 & 4.341055 & -0.824731 \\ 6 & 1.180217 & 4.218607 & -0.381143 \\ 1 & 1.331268 & 5.294740 & -0.389358 \\ 6 & 2.275912 & 3.359509 & -0.203300 \\ 1 & 3.289757 & 3.741855 & -0.137706 \\ 6 & 2.039497 & 1.996472 & -0.186526 \\ 6 & 3.122928 & 0.977132 & -0.399657 \\ 1 & 3.275177 & 0.942884 & -1.487665 \\ 1 & 4.076780 & 1.307742 & 0.039371\end{array}$

$\begin{array}{lllll}6 & 0.183624 & -2.410723 & -0.179888\end{array}$

$6 \quad-3.047379 \quad-0.867921-1.126080$

$6 \quad-3.620390-2.049725-0.341319$

$1 \quad-4.254505-2.647439-1.006775$

$\begin{array}{llll}1 & -2.833411 & -2.709152 & 0.039619\end{array}$

$1 \quad-4.244168 \quad-1.7346630 .499582$

$\begin{array}{llll}6 & -4.176845 & 0.062627 & -1.577391\end{array}$

$1 \quad-4.889989-0.514692-2.178435$

$1 \quad-4.734161 \quad 0.508500 \quad-0.751866$

$1 \quad-3.7997330 .869204 \quad-2.212941$

$6 \quad-2.376660 \quad-1.419621 \quad-2.388697$

$1 \quad-1.975707 \quad-0.624984-3.022396$

$1 \quad-1.559233-2.106279-2.159555$

$1 \quad-3.133059-1.962480-2.969296$

$\begin{array}{lllll}6 & -2.438806 & 0.420795 & 1.644456\end{array}$

$\begin{array}{lllll}6 & -2.346059 & -0.817450 & 2.540904\end{array}$

$\begin{array}{lllll}1 & -2.988295 & -1.635899 & 2.207239\end{array}$

$1 \quad-1.318691 \quad-1.189846 \quad 2.586074$

$1 \quad-2.659752 \quad-0.549050 \quad 3.557481$

$\begin{array}{llll}6 & -3.870943 & 0.952530 & 1.626423\end{array}$

$1 \quad-4.140866 \quad 1.289881 \quad 2.634991$

$1 \quad-3.979412 \quad 1.8118690 .955453$

$1 \quad-4.603040 \quad 0.193591 \quad 1.336324$

$\begin{array}{llll}6 & -1.539134 & 1.508975 & 2.242463\end{array}$

$\begin{array}{llll}1 & -0.480943 & 1.231388 & 2.196954\end{array}$

$\begin{array}{llll}1 & -1.667384 & 2.471067 & 1.737548\end{array}$

$\begin{array}{llll}1 & -1.807483 & 1.649283 & 3.296548\end{array}$

$\begin{array}{lllll}6 & 3.209742 & -0.556164 & 1.447530\end{array}$

$1 \quad 2.611482 \quad 0.158353 \quad 2.022784$

$\begin{array}{lllll}1 & 4.263121 & -0.239304 & 1.534879\end{array}$

$\begin{array}{lllll}6 & 3.048545 & -1.950497 & 2.004422\end{array}$

$13.735436 \quad-2.664041 \quad 1.539092$

$1 \quad 3.268412 \quad-1.941170 \quad 3.075025$

$\begin{array}{lllll}1 & 2.025583 & -2.310624 & 1.869973\end{array}$

$\begin{array}{lllll}6 & 3.435414 & -1.394348 & -0.862664\end{array}$

$1 \quad 3.060838 \quad-2.379261 \quad-0.569604$

$1 \quad 3.040388-1.206241-1.866327$

$\begin{array}{lllll}6 & 4.953517 & -1.375932 & -0.863664\end{array}$

$1 \quad 5.333101 \quad-2.115549-1.572945$

$1 \quad 5.355842 \quad-0.402598 \quad-1.162699$

$1 \quad 5.370865 \quad-1.6223420 .117904$

$1 \quad-2.294557 \quad 2.195471 \quad-1.278530$

$1 \quad-0.676213 \quad 0.721590 \quad-1.865910$ 


\section{B-TS2 $2_{0}$-PS}

$\begin{array}{llll}6 & 0.386661 & -0.406976 & 2.079625\end{array}$

$\begin{array}{lllll}6 & -0.400777 & 1.817262 & 1.979968\end{array}$

$\begin{array}{llll}7 & 0.171298 & 0.750134 & 1.394874\end{array}$

$\begin{array}{lllll}6 & -0.618259 & 0.604127 & 4.025762\end{array}$

$\begin{array}{lllll}15 & 2.011617 & -0.934171 & -0.052804\end{array}$

$\begin{array}{lllll}6 & 3.709535 & -0.612939 & 0.746609\end{array}$

$\begin{array}{lllll}6 & 2.135079 & -2.385767 & -1.268531\end{array}$

$\begin{array}{llll}6 & 3.463114 & 0.241147 & 1.996342\end{array}$

$\begin{array}{llll}1 & 4.430407 & 0.554537 & 2.406850\end{array}$

$\begin{array}{llll}1 & 2.884252 & 1.142090 & 1.770723\end{array}$

$\begin{array}{llll}1 & 2.939177 & -0.319212 & 2.776666\end{array}$

$\begin{array}{lllll}6 & 4.606177 & 0.182899 & -0.203881\end{array}$

$\begin{array}{llll}1 & 4.116024 & 1.110700 & -0.514228\end{array}$

$\begin{array}{llll}1 & 5.535910 & 0.449525 & 0.313580\end{array}$

$14.881026-0.373075-1.102904$

$\begin{array}{lllll}6 & 0.941682 & -2.338317 & -2.229695\end{array}$

$\begin{array}{lllll}1 & 1.027027 & -3.189223 & -2.917093\end{array}$

$\begin{array}{lllll}1 & -0.016637 & -2.414976 & -1.709546\end{array}$

$\begin{array}{lllll}1 & 0.947509 & -1.425401 & -2.833090\end{array}$

$\begin{array}{lllll}6 & 2.091496 & -3.724517 & -0.526496\end{array}$

$\begin{array}{lllll}1 & 2.201301 & -4.537958 & -1.253718\end{array}$

$\begin{array}{lllll}1 & 2.888874 & -3.837696 & 0.212835\end{array}$

$\begin{array}{llll}1 & 1.128590 & -3.863430 & -0.025105\end{array}$

$\begin{array}{llll}6 & -0.546745 & 5.299627 & -0.837141\end{array}$

$\begin{array}{lllll}1 & -0.010856 & 6.038477 & -1.438166\end{array}$

$1 \quad-1.6027695 .369586 \quad-1.110421$

$\begin{array}{llll}1 & -0.447779 & 5.605180 & 0.209021\end{array}$

$\begin{array}{llll}6 & -2.721894 & 1.555866 & 0.057283\end{array}$

$\begin{array}{llll}1 & -2.915783 & 1.890892 & 1.081321\end{array}$

$1 \quad-3.690976 \quad 1.376212 \quad-0.415242$

$\begin{array}{llll}1 & -2.205790 & 0.594804 & 0.104394\end{array}$

$1 \quad 2.004936 \quad 1.880316 \quad-0.032214$

$\begin{array}{llll}1 & -0.614059 & 0.179284 & -1.534461\end{array}$

$\begin{array}{lllll}1 & -0.106951 & -1.900475 & 0.681071\end{array}$

$\begin{array}{lllll}8 & -1.233243 & -1.987430 & -0.069128\end{array}$

$\begin{array}{lllll}1 & -0.643477 & -0.400213 & -0.953819\end{array}$

$\begin{array}{llll}6 & -2.357479 & -2.023083 & 0.730610\end{array}$

$\begin{array}{llll}6 & -2.501239 & -3.385234 & 1.418013\end{array}$$$
1
$$$$
1
$$

$$
1
$$

$$
6
$$

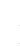$$
6
$$$$
1
$$

$$
1
$$

$$
1
$$$$
1
$$

1

6

1

6

1

6

1
1

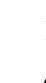

6

$\begin{array}{lllll}1 & 4.326323 & -2.410678 & -1.553673\end{array}$

$\begin{array}{lllll}1 & 3.375375 & -3.060371 & -2.885261\end{array}$

$1 \quad 3.455894 \quad-1.315699 \quad-2.645422$

$\begin{array}{lllll}6 & 4.405056 & -1.895780 & 1.203755\end{array}$

$\begin{array}{lllll}1 & 4.721804 & -2.536668 & 0.377670\end{array}$

$\begin{array}{lllll}1 & 5.306393 & -1.632213 & 1.770782\end{array}$

$\begin{array}{lllll}1 & 3.767449 & -2.488505 & 1.869344\end{array}$

$\begin{array}{lllll}6 & 0.049133 & 3.926036 & -1.092769\end{array}$

$\begin{array}{lllll}1 & -0.041793 & 3.656824 & -2.150254\end{array}$

$\begin{array}{lllll}1 & 1.118805 & 3.927305 & -0.863149\end{array}$

$\begin{array}{lllll}6 & -1.953837 & 2.578384 & -0.746992\end{array}$

$\begin{array}{lllll}1 & -1.909222 & 2.281614 & -1.801146\end{array}$

$\begin{array}{lllll}1 & -2.483000 & 3.542493 & -0.703874\end{array}$

$\begin{array}{lllll}6 & 1.630034 & 1.205372 & -2.273808\end{array}$

$\begin{array}{lllll}8 & 2.197858 & 1.378300 & -3.284351\end{array}$

$\begin{array}{lllll}44 & 0.832149 & 0.957607 & -0.630261\end{array}$

$\begin{array}{lllll}6 & 0.933455 & -1.513762 & 1.308575\end{array}$

$\begin{array}{lllll}1 & 1.342604 & -2.320823 & 1.924659\end{array}$

$\begin{array}{llll}7 & -0.538189 & 2.789216 & -0.322819\end{array}$ 


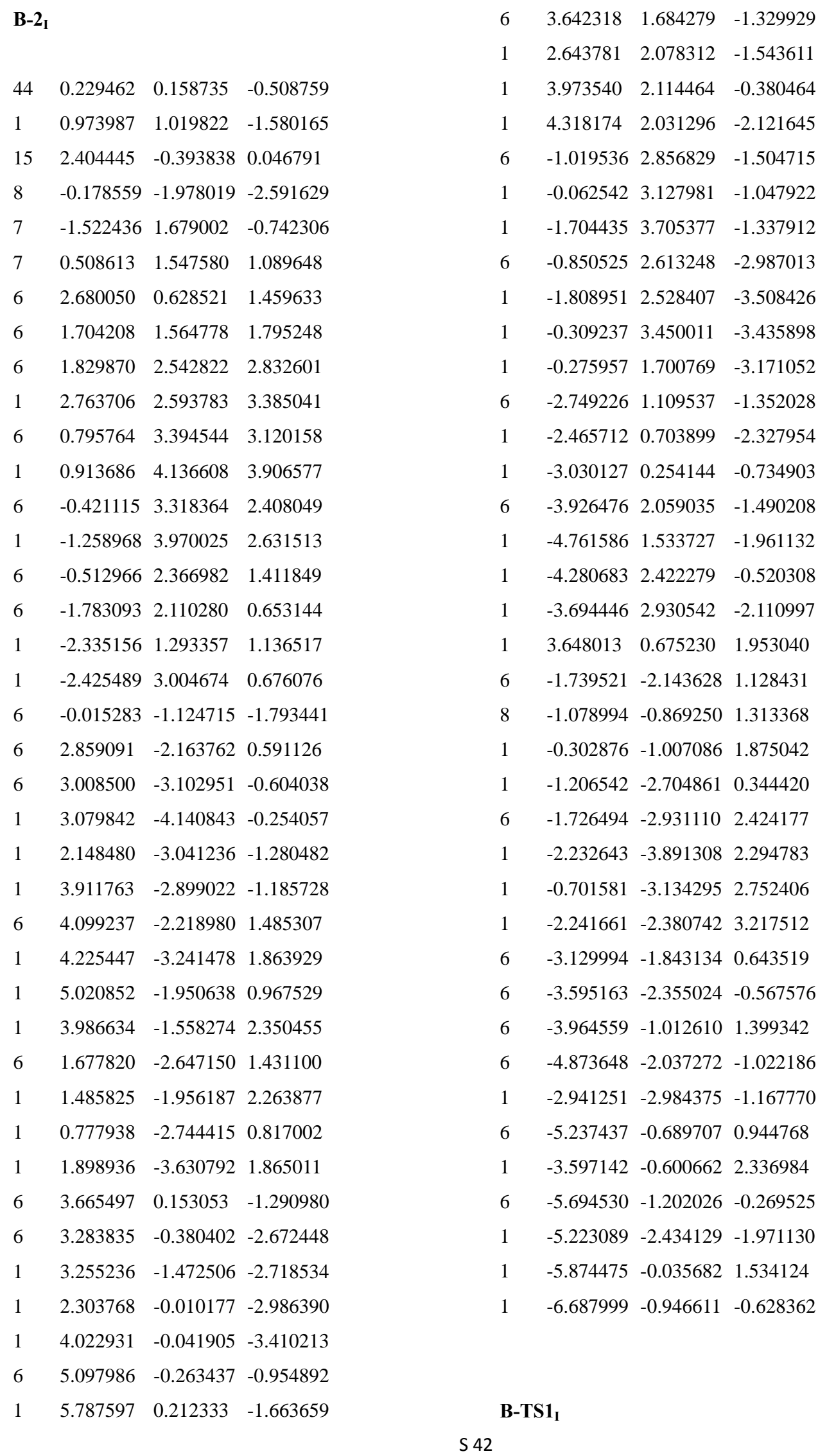

$\begin{array}{lllll}6 & 3.642318 & 1.684279 & -1.329929\end{array}$

$1 \quad 2.643781 \quad 2.078312 \quad-1.543611$

$\begin{array}{lllll}1 & 3.973540 & 2.114464 & -0.380464\end{array}$

$\begin{array}{lllll}1 & 4.318174 & 2.031296 & -2.121645\end{array}$

$\begin{array}{lllll}6 & -1.019536 & 2.856829 & -1.504715\end{array}$

$\begin{array}{lllll}1 & -0.062542 & 3.127981 & -1.047922\end{array}$

$\begin{array}{lllll}1 & -1.704435 & 3.705377 & -1.337912\end{array}$

$\begin{array}{lllll}6 & -0.850525 & 2.613248 & -2.987013\end{array}$

$\begin{array}{lllll}1 & -1.808951 & 2.528407 & -3.508426\end{array}$

$\begin{array}{llll}1 & -0.309237 & 3.450011 & -3.435898\end{array}$

$\begin{array}{lllll}1 & -0.275957 & 1.700769 & -3.171052\end{array}$

$\begin{array}{lllll}6 & -2.749226 & 1.109537 & -1.352028\end{array}$

$\begin{array}{lllll}1 & -2.465712 & 0.703899 & -2.327954\end{array}$

$\begin{array}{lllll}1 & -3.030127 & 0.254144 & -0.734903\end{array}$

$\begin{array}{lllll}6 & -3.926476 & 2.059035 & -1.490208\end{array}$

$1 \quad-4.761586 \quad 1.533727 \quad-1.961132$

$\begin{array}{lllll}1 & -4.280683 & 2.422279 & -0.520308\end{array}$

$\begin{array}{llll}1 & -3.694446 & 2.930542 & -2.110997\end{array}$

$\begin{array}{llll}1 & 3.648013 & 0.675230 & 1.953040\end{array}$

$\begin{array}{lllll}6 & -1.739521 & -2.143628 & 1.128431\end{array}$

$\begin{array}{lllll}8 & -1.078994 & -0.869250 & 1.313368\end{array}$

$1-0.302876-1.0070861 .875042$

$\begin{array}{lllll}1 & -1.206542 & -2.704861 & 0.344420\end{array}$

$\begin{array}{lllll}6 & -1.726494 & -2.931110 & 2.424177\end{array}$

$\begin{array}{lllll}1 & -2.232643 & -3.891308 & 2.294783\end{array}$

$\begin{array}{llll}1 & -0.701581 & -3.134295 & 2.752406\end{array}$

$\begin{array}{llll}1 & -2.241661 & -2.380742 & 3.217512\end{array}$

$\begin{array}{lllll}6 & -3.129994 & -1.843134 & 0.643519\end{array}$

$\begin{array}{lllll}6 & -3.595163 & -2.355024 & -0.567576\end{array}$

$\begin{array}{lllll}6 & -3.964559 & -1.012610 & 1.399342\end{array}$

$6 \quad-4.873648 \quad-2.037272 \quad-1.022186$

$1 \quad-2.941251-2.984375-1.167770$

$\begin{array}{lllll}6 & -5.237437 & -0.689707 & 0.944768\end{array}$

$\begin{array}{lllll}1 & -3.597142 & -0.600662 & 2.336984\end{array}$

$\begin{array}{lllll}6 & -5.694530 & -1.202026 & -0.269525\end{array}$

$1 \quad-5.223089-2.434129-1.971130$

$\begin{array}{llll}1 & -5.874475 & -0.035682 & 1.534124\end{array}$

$\begin{array}{lllll}1 & -6.687999 & -0.946611 & -0.628362\end{array}$

B-TS1

$\begin{array}{llll}5.392104 & 0.059828 & 0.049841\end{array}$

$\begin{array}{llll}5.256827 & -1.342542 & -1.031593\end{array}$ 


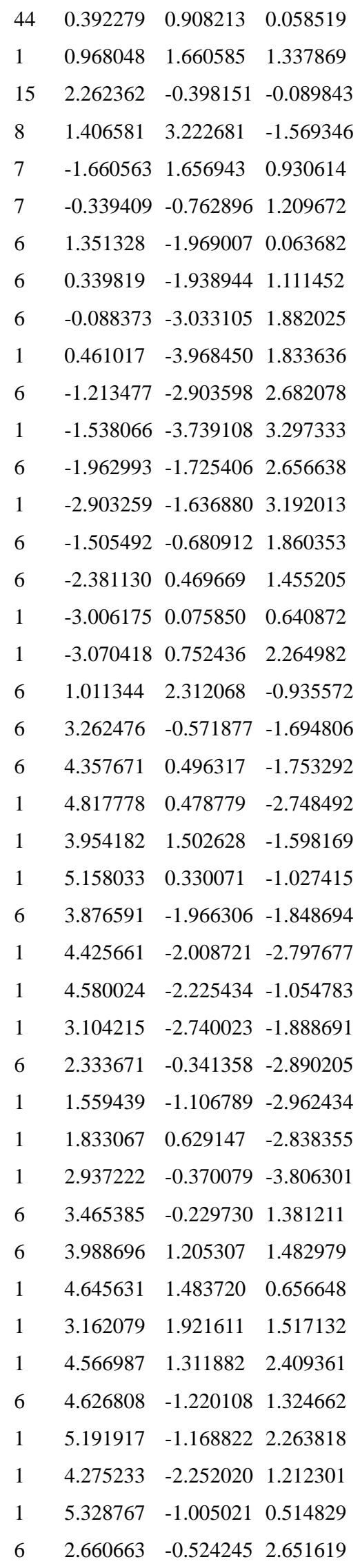

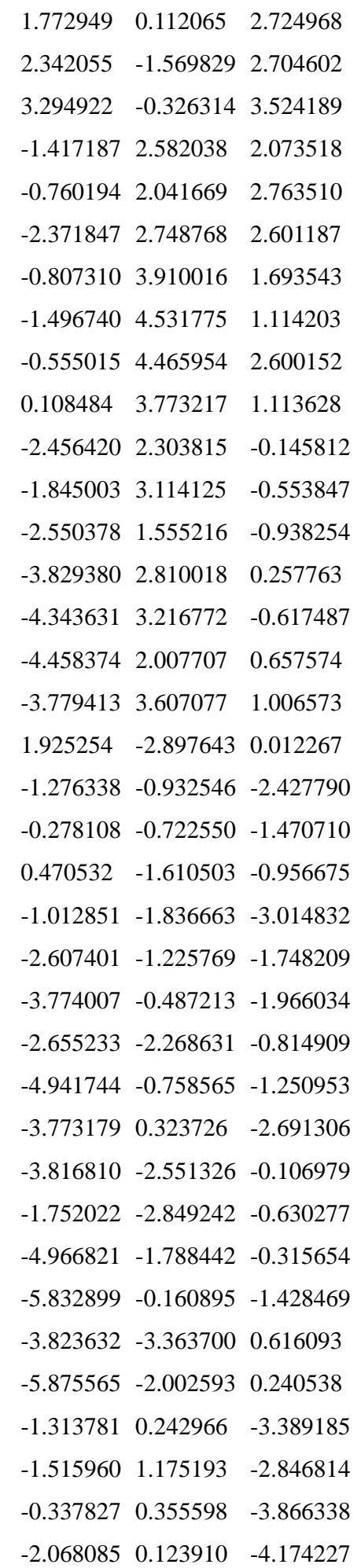



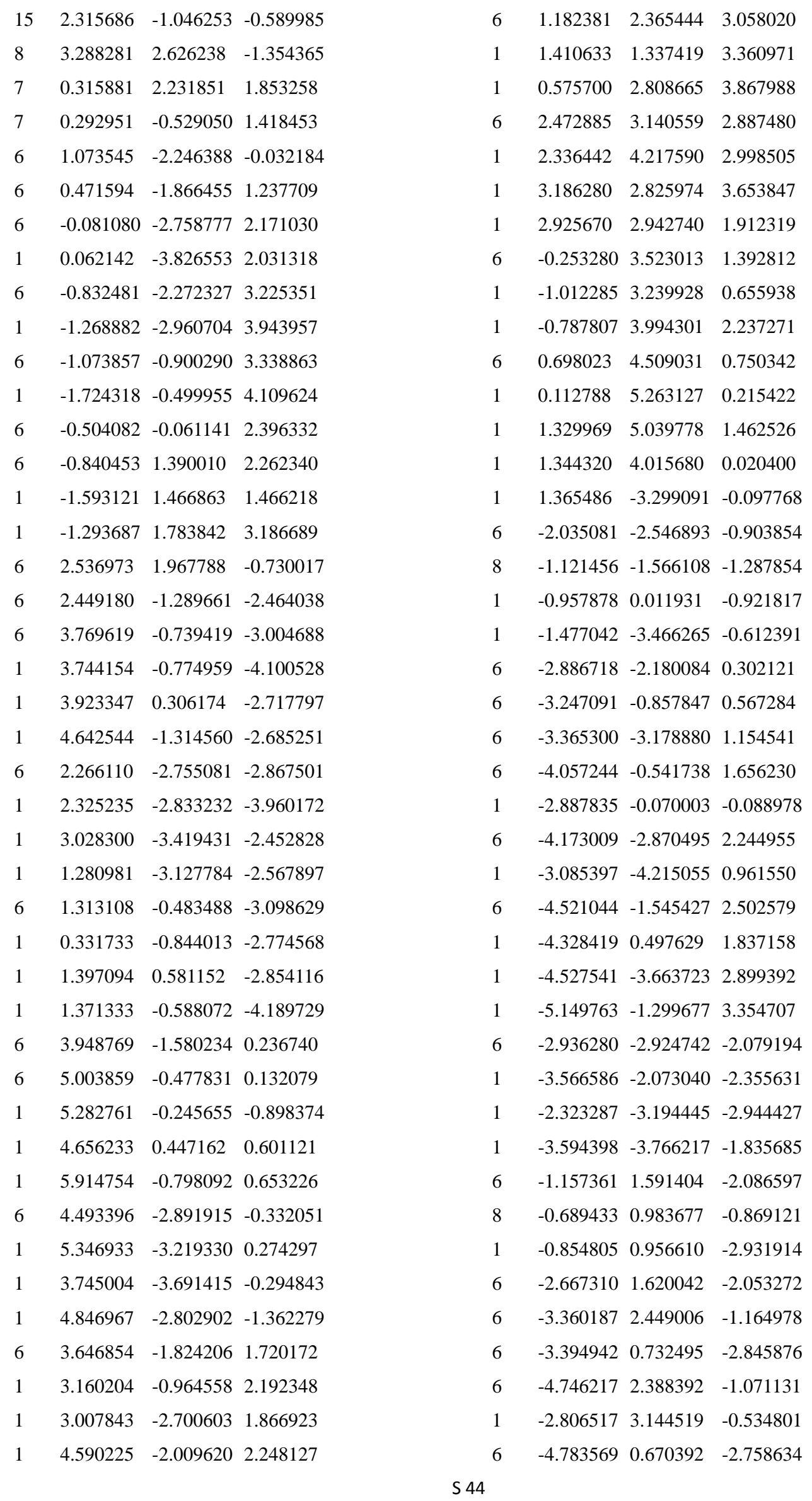


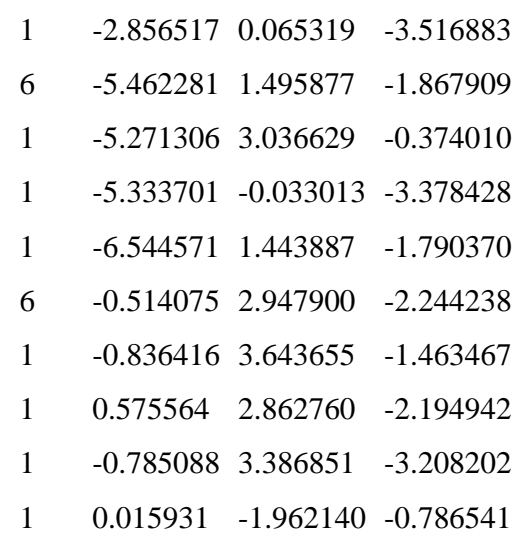

B-3 I $_{\text {I }}$

$\begin{array}{llll}44 & 0.450753 & 0.377686 & -0.709316 \\ 1 & 1.304455 & 1.643512 & -1.243888 \\ 15 & 2.398265 & -0.336000 & 0.174373 \\ 8 & 0.877261 & -0.775867 & -3.450100 \\ 7 & -1.492558 & 1.675381 & -0.891177 \\ 7 & 0.319014 & 1.428399 & 1.161349 \\ 6 & 2.675668 & 0.994513 & 1.457175 \\ 6 & 1.381527 & 1.530716 & 1.984881 \\ 6 & 1.251505 & 2.133988 & 3.233367 \\ 1 & 2.109843 & 2.193066 & 3.895426 \\ 6 & 0.018057 & 2.658303 & 3.606036 \\ 1 & -0.102667 & 3.133529 & 4.575245 \\ 6 & -1.053730 & 2.584895 & 2.724088 \\ 1 & -2.022983 & 3.003051 & 2.978792 \\ 6 & -0.873858 & 1.952711 & 1.496432 \\ 6 & -1.979664 & 1.753525 & 0.507146 \\ 1 & -2.451571 & 0.788733 & 0.728816 \\ 1 & -2.732264 & 2.549521 & 0.619151 \\ 6 & 0.678532 & -0.364575 & -2.365552 \\ 6 & 2.353880 & -1.914473 & 1.234033 \\ 6 & 1.817520 & -3.062738 & 0.380461 \\ 1 & 1.679799 & -3.950041 & 1.010410 \\ 1 & 0.846843 & -2.795805 & -0.041296 \\ 1 & 2.487778 & -3.340101 & -0.437759 \\ 6 & 3.682446 & -2.315540 & 1.870130 \\ 1 & 3.505870 & -3.155054 & 2.554261 \\ 1 & 4.422888 & -2.651548 & 1.137809 \\ 1 & 4.128094 & -1.508952 & 2.463518 \\ 6 & 1.332689 & -1.642399 & 2.348158\end{array}$

$1 \quad 1.697654 \quad-0.905027 \quad 3.072529$

$1 \quad 0.371156 \quad-1.3109501 .931759$

$\begin{array}{llll}1 & 1.163963 & -2.575372 & 2.900584\end{array}$

$6 \quad 4.004848 \quad-0.275415 \quad-0.832360$

$6 \quad 4.145051 \quad-1.568810-1.637188$

$1 \quad 4.371385-2.432862 \quad-1.005922$

$1 \quad 3.241514 \quad-1.790410 \quad-2.215032$

$14.971525 \quad-1.463103 \quad-2.350093$

$\begin{array}{lllll}6 & 5.251453 & -0.055657 & 0.031387\end{array}$

$1 \quad 6.133335 \quad-0.038467-0.620133$

$\begin{array}{llll}1 & 5.224567 & 0.907565 & 0.551225\end{array}$

$\begin{array}{lllll}1 & 5.413600 & -0.836893 & 0.774724\end{array}$

$\begin{array}{lllll}6 & 3.914487 & 0.893918 & -1.816931\end{array}$

$1 \quad 3.111515 \quad 0.760364 \quad-2.544176$

$1 \quad 3.741658 \quad 1.851146 \quad-1.313231$

$\begin{array}{lllll}1 & 4.866026 & 0.967937 & -2.357991\end{array}$

$\begin{array}{lllll}6 & -1.195113 & 3.045335 & -1.376661\end{array}$

$\begin{array}{llll}1 & -0.393950 & 3.429886 & -0.735115\end{array}$

$\begin{array}{lllll}1 & -2.076687 & 3.687919 & -1.209022\end{array}$

$\begin{array}{llll}6 & -0.774954 & 3.104956 & -2.826503\end{array}$

$\begin{array}{llll}1 & -1.602767 & 2.885203 & -3.506949\end{array}$

$\begin{array}{llll}1 & -0.414525 & 4.108928 & -3.064983\end{array}$

$\begin{array}{lllll}1 & 0.035228 & 2.396246 & -3.021217\end{array}$

$\begin{array}{lllll}6 & -2.498743 & 0.973171 & -1.728751\end{array}$

$\begin{array}{lllll}1 & -2.064124 & 0.865196 & -2.727237\end{array}$

$1 \quad-2.571244-0.028965-1.301429$

$6 \quad-3.872066 \quad 1.614254 \quad-1.795152$

$\begin{array}{lllll}1 & -4.519801 & 1.013834 & -2.439456\end{array}$

$\begin{array}{llll}1 & -4.352032 & 1.647732 & -0.811604\end{array}$

$\begin{array}{llll}1 & -3.850867 & 2.630528 & -2.203506\end{array}$

$\begin{array}{llll}1 & 3.190183 & 1.804330 & 0.921734\end{array}$

$\begin{array}{lllll}6 & -1.657998 & -2.088447 & -0.005544\end{array}$

$\begin{array}{lllll}8 & -1.002858 & -0.912682 & 0.319200\end{array}$

$1 \quad-1.297800-2.9230050 .649685$

$\begin{array}{llll}1 & 3.340707 & 0.680815 & 2.271586\end{array}$

$6 \quad-3.140575 \quad-1.909166 \quad 0.332630$

$6 \quad-4.188115 \quad-2.196391 \quad-0.546447$

$6 \quad-3.466729 \quad-1.362308 \quad 1.581697$

$6 \quad-5.514295-1.936577-0.197690$

$1 \quad-3.973880-2.617875 \quad-1.525644$

$6 \quad-4.785939-1.110051 \quad 1.938438$

$1 \quad-2.650579 \quad-1.1203892 .261191$

$\begin{array}{llll}6 & -5.820041 & -1.393630 & 1.045050\end{array}$ 
$1 \quad-6.310177-2.160204-0.904593$

$\begin{array}{llll}1 & -5.012924 & -0.691379 & 2.916647\end{array}$

$1 \quad-6.852772 \quad-1.193504 \quad 1.318433$

$6 \quad-1.445068-2.572223-1.437210$

$1 \quad-1.930457-3.538542-1.618934$

$1 \quad-1.829092-1.854337-2.172154$

$\begin{array}{llll}1 & -0.378605 & -2.693834 & -1.642573\end{array}$

\section{B-TS2 I}

$44 \quad 0.118651 \quad-0.517244 \quad-0.855925$

$\begin{array}{lllll}1 & 0.647422 & -2.013221 & -1.138937\end{array}$

$\begin{array}{lllll}15 & -1.490230 & -1.649380 & 0.161182\end{array}$

$\begin{array}{lllll}8 & -1.267447 & -0.638525 & -3.505621\end{array}$

$\begin{array}{lllll}7 & 4.054537 & -0.307467 & 0.015752\end{array}$

$\begin{array}{lllll}7 & 1.126188 & -0.653418 & 1.199718\end{array}$

$\begin{array}{lllll}6 & -0.664528 & -2.254059 & 1.696234\end{array}$

$\begin{array}{lllll}6 & 0.468401 & -1.377147 & 2.138514\end{array}$

$\begin{array}{lllll}6 & 0.838282 & -1.358896 & 3.480837\end{array}$

$\begin{array}{llll}1 & 0.264801 & -1.937687 & 4.198603\end{array}$

$\begin{array}{llll}6 & 1.930636 & -0.599457 & 3.875865\end{array}$

$1 \quad 2.228343 \quad-0.557796 \quad 4.919751$

$\begin{array}{llll}6 & 2.639497 & 0.088726 & 2.905192\end{array}$

$\begin{array}{llll}1 & 3.519532 & 0.671785 & 3.159849\end{array}$

$\begin{array}{llll}6 & 2.228892 & 0.039447 & 1.571917\end{array}$

$\begin{array}{llll}6 & 3.087332 & 0.678704 & 0.513670\end{array}$

$\begin{array}{llll}1 & 2.456163 & 1.083874 & -0.281978\end{array}$

$\begin{array}{llll}1 & 3.615722 & 1.531028 & 0.959689\end{array}$

$\begin{array}{lllll}6 & -0.714715 & -0.561507 & -2.470867\end{array}$

$\begin{array}{llll}6 & -2.822522 & -0.441045 & 0.784515\end{array}$

$\begin{array}{lllll}6 & -3.353379 & 0.366895 & -0.403739\end{array}$

$\begin{array}{llll}1 & -4.072439 & 1.111064 & -0.040840\end{array}$

$\begin{array}{lllll}1 & -2.543107 & 0.910682 & -0.900488\end{array}$

$1 \quad-3.859341 \quad-0.249797 \quad-1.151596$

$\begin{array}{lllll}6 & -3.978057 & -1.099417 & 1.535022\end{array}$

$1 \quad-4.626466 \quad-0.316643 \quad 1.946951$

$\begin{array}{llll}1 & -4.602055 & -1.726523 & 0.892011\end{array}$

$\begin{array}{llll}1 & -3.636511 & -1.707627 & 2.380657\end{array}$

$\begin{array}{lllll}6 & -2.111867 & 0.533951 & 1.731461\end{array}$

$\begin{array}{llll}1 & -1.807294 & 0.056380 & 2.670023\end{array}$

$\begin{array}{llll}1 & -1.233372 & 0.991683 & 1.261372\end{array}$

$\begin{array}{llll}1 & -2.801075 & 1.348322 & 1.985977\end{array}$ $\begin{array}{llll}1 & -3.016372 & -2.202720 & -2.328647\end{array}$

$1 \quad-3.724887 \quad-3.790870 \quad-2.029611$

$\begin{array}{lllll}6 & -2.870888 & -4.112031 & 0.573715\end{array}$

$\begin{array}{llll}1 & -3.326147 & -4.996423 & 0.112176\end{array}$

$\begin{array}{llll}1 & -2.100769 & -4.473457 & 1.262337\end{array}$

$1 \quad-3.646445 \quad-3.617652 \quad 1.161235$

$6 \quad-1.198762-4.020435-1.256390$

$1 \quad-0.810501 \quad-3.480546 \quad-2.122689$

$1 \quad-0.346912-4.250877-0.607230$

$1 \quad-1.625516-4.971308-1.597699$

$\begin{array}{lllll}6 & 5.433567 & 0.115829 & 0.222541\end{array}$

$\begin{array}{llll}1 & 5.545569 & 0.331561 & 1.293565\end{array}$

$\begin{array}{lllll}1 & 5.663236 & 1.067487 & -0.300223\end{array}$

$\begin{array}{lllll}6 & 6.443207 & -0.938397 & -0.180136\end{array}$

$1 \quad 6.468649 \quad-1.091067-1.263389$

$\begin{array}{llll}1 & 7.449020 & -0.641893 & 0.128772\end{array}$

$\begin{array}{lllll}1 & 6.208035 & -1.898950 & 0.288079\end{array}$

$6 \quad 3.739192 \quad-0.738393-1.346272$

$1 \quad 4.321926-1.640376-1.567587$

$1 \quad 2.687395 \quad-1.063952-1.340478$

$\begin{array}{lllll}6 & 3.947395 & 0.304145 & -2.435457\end{array}$

$\begin{array}{lllll}1 & 3.617718 & -0.080370 & -3.404997\end{array}$

$\begin{array}{lllll}1 & 3.370360 & 1.213934 & -2.233462\end{array}$

$1 \quad 4.999796 \quad 0.592784 \quad-2.533138$

$\begin{array}{lllll}1 & -0.250409 & -3.235283 & 1.425302\end{array}$

$\begin{array}{lllll}6 & 0.962456 & 3.364472 & -2.043849\end{array}$

$\begin{array}{lllll}1 & 1.532959 & 2.748857 & -2.747146\end{array}$

$1 \quad 1.652363 \quad 3.745740 \quad-1.282007$

$\begin{array}{lllll}6 & -0.127392 & 2.525434 & -1.382826\end{array}$

$\begin{array}{lllll}8 & 0.455046 & 1.483511 & -0.657331\end{array}$

$1 \quad-1.365460 \quad-2.429596 \quad 2.521670$

$\begin{array}{llll}1 & -0.790411 & 2.142500 & -2.184921\end{array}$

$\begin{array}{lllll}6 & -0.985150 & 3.374380 & -0.463892\end{array}$

$\begin{array}{lllll}6 & -2.229357 & 3.849065 & -0.880111\end{array}$

$\begin{array}{lllll}6 & -0.540726 & 3.695390 & 0.821757\end{array}$

$\begin{array}{lllll}6 & -3.017410 & 4.628599 & -0.036117\end{array}$

$\begin{array}{llll}1 & -2.587012 & 3.590057 & -1.876577\end{array}$

$\begin{array}{llll}6 & -1.321773 & 4.476321 & 1.667452\end{array}$

$1 \quad 0.417316 \quad 3.297870 \quad 1.153145$

$\begin{array}{llll}6 & -2.564492 & 4.945395 & 1.241272\end{array}$ 


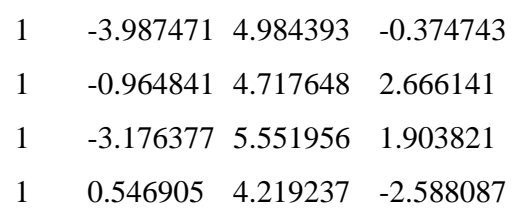

\section{B-4}

$44 \quad-0.305054-0.659457-0.959576$

$1 \quad-0.333183 \quad-2.258303 \quad-1.180071$

$\begin{array}{lllll}15 & -2.124797 & -1.060435 & 0.237365\end{array}$

$8 \quad-1.821172 \quad-0.396748 \quad-3.530680$

$\begin{array}{lllll}7 & 4.239331 & -1.041292 & 0.178463\end{array}$

$\begin{array}{lllll}7 & 0.726938 & -1.014829 & 1.012847\end{array}$

$\begin{array}{lllll}6 & -1.450998 & -1.759517 & 1.814660\end{array}$

$\begin{array}{llll}6 & -0.048613 & -1.302051 & 2.084217\end{array}$

$\begin{array}{lllll}6 & 0.446288 & -1.229006 & 3.383058\end{array}$

$\begin{array}{lllll}1 & -0.209997 & -1.451988 & 4.219125\end{array}$

$\begin{array}{lllll}6 & 1.776385 & -0.879111 & 3.582951\end{array}$

$\begin{array}{lllll}1 & 2.180882 & -0.806623 & 4.588787\end{array}$

$\begin{array}{llll}6 & 2.582213 & -0.647864 & 2.479353\end{array}$

$\begin{array}{llll}1 & 3.640824 & -0.424092 & 2.564814\end{array}$

$\begin{array}{lllll}6 & 2.032066 & -0.727738 & 1.200765\end{array}$

$\begin{array}{lllll}6 & 2.898135 & -0.516794 & -0.009179\end{array}$

$\begin{array}{lllll}1 & 2.373203 & -0.920905 & -0.897473\end{array}$

$\begin{array}{lllll}1 & 2.967283 & 0.558978 & -0.204179\end{array}$

$\begin{array}{lllll}6 & -1.209844 & -0.475418 & -2.529552\end{array}$

$\begin{array}{lllll}6 & -2.897498 & 0.593867 & 0.778586\end{array}$

$\begin{array}{lllll}6 & -3.150318 & 1.456533 & -0.461422\end{array}$

$\begin{array}{lllll}1 & -3.581412 & 2.415947 & -0.150539\end{array}$

$1 \quad-2.213944 \quad 1.669140 \quad-0.986631$

$\begin{array}{lllll}1 & -3.839277 & 0.995091 & -1.174765\end{array}$

$\begin{array}{llll}6 & -4.178686 & 0.459174 & 1.598917\end{array}$

$\begin{array}{llll}1 & -4.470418 & 1.450872 & 1.965874\end{array}$

$\begin{array}{llll}1 & -5.021302 & 0.072084 & 1.018521\end{array}$

$\begin{array}{lllll}1 & -4.044125 & -0.180273 & 2.478863\end{array}$

$\begin{array}{llll}6 & -1.841736 & 1.302546 & 1.637398\end{array}$

$\begin{array}{llll}1 & -1.703661 & 0.816270 & 2.609832\end{array}$

$\begin{array}{llll}1 & -0.872735 & 1.363358 & 1.127131\end{array}$

$\begin{array}{llll}1 & -2.173878 & 2.329588 & 1.832381\end{array}$

$6 \quad-3.466190 \quad-2.302703 \quad-0.250848$

$6 \quad-4.396118-1.679605 \quad-1.294041$

$\begin{array}{lllll}1 & -5.009606 & -0.873707 & -0.881148\end{array}$
$1 \quad-3.844510 \quad-1.286891-2.153476$

$1 \quad-5.081373-2.449179-1.668712$

$\begin{array}{lllll}6 & -4.284232 & -2.800699 & 0.945997\end{array}$

$1 \quad-5.021483-3.5294780 .588545$

$1 \quad-3.663447 \quad-3.317112 \quad 1.684986$

$1 \quad-4.835473 \quad-2.0102961 .457501$

$6 \quad-2.772872-3.512995-0.884657$

$1 \quad-2.261456-3.250684-1.813120$

$1 \quad-2.028471 \quad-3.964320 \quad-0.219378$

$1 \quad-3.528935-4.276385-1.104514$

$\begin{array}{lllll}6 & 5.129535 & -0.511417 & -0.852557\end{array}$

$1 \quad 5.062997 \quad 0.582262 \quad-0.790412$

$14.782303 \quad-0.771231-1.872178$

$\begin{array}{lllll}6 & 6.570023 & -0.943414 & -0.677097\end{array}$

$1 \quad 6.715120 \quad-2.006553 \quad-0.892065$

$1 \quad 7.218675 \quad-0.385419-1.356898$

$\begin{array}{lllll}1 & 6.909073 & -0.757503 & 0.346641\end{array}$

$\begin{array}{lllll}6 & 4.262605 & -2.504198 & 0.286924\end{array}$

$1 \quad 5.272014 \quad-2.7993530 .595392$

$1 \quad 3.609186 \quad-2.773966 \quad 1.125969$

$6 \quad 3.846490 \quad-3.282513 \quad-0.954350$

$1 \quad 3.892043 \quad-4.357928 \quad-0.761745$

$1 \quad 2.821283 \quad-3.048999-1.259759$

$14.502570 \quad-3.075229 \quad-1.806264$

$1 \quad-1.436506-2.8483491 .668096$

$\begin{array}{llll}6 & 0.546789 & 2.286488 & -1.536435\end{array}$

$\begin{array}{llll}1 & -0.335261 & 2.245761 & -2.208456\end{array}$

$8 \quad 0.701532 \quad 1.104049-0.813773$

$1 \quad-2.100868-1.5672542 .677179$

$\begin{array}{lllll}6 & 0.333356 & 3.435928 & -0.568480\end{array}$

$6 \quad-0.594053 \quad 4.448414 \quad-0.816355$

$\begin{array}{llll}6 & 1.075679 & 3.481610 & 0.615456\end{array}$

$\begin{array}{llll}6 & -0.777825 & 5.486120 & 0.096266\end{array}$

$\begin{array}{llll}-1.186635 & 4.412842 & -1.730287\end{array}$

$\begin{array}{lllll}6 & 0.899680 & 4.516375 & 1.526291\end{array}$

$\begin{array}{lllll}1 & 1.773961 & 2.672080 & 0.820298\end{array}$

$\begin{array}{llll}6 & -0.030701 & 5.523351 & 1.270162\end{array}$

$1 \quad-1.510587 \quad 6.263225 \quad-0.107506$

$1 \quad 1.483654 \quad 4.537653 \quad 2.443400$

$1 \quad-0.175063 \quad 6.329800 \quad 1.984443$

$\begin{array}{llll}6 & 1.779894 & 2.520819 & -2.406218\end{array}$

$1 \quad 2.672582 \quad 2.605877 \quad-1.775197$

$1 \quad 1.920983 \quad 1.673230 \quad-3.085528$ 


\section{B-TS3}

$\begin{array}{llll}44 & 0.311687 & 0.296371 & 0.330191\end{array}$

$\begin{array}{lllll}1 & -0.377170 & -0.104241 & 1.717996\end{array}$

$\begin{array}{lllll}15 & 1.427639 & -1.729232 & 0.217955\end{array}$

$\begin{array}{llll}8 & 2.397917 & 1.537765 & 2.064000\end{array}$

$\begin{array}{llll}7 & -4.476121 & 0.154714 & 0.204750\end{array}$

$7 \quad-1.380700-1.030240-0.684723$

$\begin{array}{lllll}6 & 0.132788 & -2.916973 & -0.340282\end{array}$

$\begin{array}{lllll}6 & -0.983407 & -2.262254 & -1.094803\end{array}$

$\begin{array}{lllll}6 & -1.665389 & -2.964888 & -2.083940\end{array}$

$1 \quad-1.289067-3.931934-2.404716$

$\begin{array}{lllll}6 & -2.829693 & -2.431107 & -2.622538\end{array}$

$1 \quad-3.368315-2.954927-3.407072$

$6 \quad-3.317491-1.249289-2.090190$

$1 \quad-4.278166 \quad-0.838580-2.383621$

$6 \quad-2.579834-0.579987-1.112285$

$\begin{array}{lllll}6 & -3.220240 & 0.584825 & -0.408498\end{array}$

$\begin{array}{llll}1 & -2.499407 & 1.021758 & 0.303603\end{array}$

$\begin{array}{llll}1 & -3.480998 & 1.370503 & -1.128122\end{array}$

$\begin{array}{llll}6 & 1.585236 & 1.075343 & 1.353056\end{array}$

$6 \quad 2.695636-1.800850-1.190076$

$\begin{array}{lllll}6 & 3.599837 & -0.569868 & -1.074568\end{array}$

$14.309943 \quad-0.561564-1.910604$

$\begin{array}{llll}1 & 3.004106 & 0.346970 & -1.118586\end{array}$

$14.181845 \quad-0.558000-0.147612$

$6 \quad 3.538733 \quad-3.072860-1.247963$

$1 \quad 4.154249 \quad-3.048377-2.155746$

$1 \quad 4.222453 \quad-3.173571-0.400266$

$\begin{array}{lllll}1 & 2.923241 & -3.978320 & -1.303528\end{array}$

$6 \quad \begin{array}{llll}6 & 1.899453 & -1.699590 & -2.498135\end{array}$

$1 \quad 1.347101 \quad-2.621316 \quad-2.713549$

$1 \quad 1.202460 \quad-0.857273-2.498253$

$\begin{array}{lllll}1 & 2.602554 & -1.547412 & -3.325889\end{array}$

$\begin{array}{lllll}6 & 2.112463 & -2.563482 & 1.774506\end{array}$

$\begin{array}{lllll}6 & 3.460937 & -1.938780 & 2.137658\end{array}$

$1 \quad 4.243661 \quad-2.181063 \quad 1.412684$

$\begin{array}{lllll}1 & 3.398920 & -0.849852 & 2.223244\end{array}$

$\begin{array}{llll}1 & 3.788897 & -2.325510 & 3.110103\end{array}$

$\begin{array}{lllll}6 & 2.257099 & -4.082544 & 1.644831\end{array}$

$\begin{array}{llll}1 & 2.622879 & -4.486239 & 2.596646\end{array}$
$1 \quad 1.299790 \quad-4.573448 \quad 1.441797$

$1 \quad 2.964485-4.3863890 .872005$

$\begin{array}{lllll}6 & 1.122926 & -2.275560 & 2.908333\end{array}$

$1 \quad 1.065667 \quad-1.209483 \quad 3.136263$

$\begin{array}{lllll}1 & 0.105397 & -2.609601 & 2.671399\end{array}$

$\begin{array}{lllll}1 & 1.446745 & -2.810954 & 3.809047\end{array}$

$\begin{array}{llll}6 & -5.214244 & 1.323866 & 0.679446\end{array}$

$1 \quad-5.349239 \quad 1.982188 \quad-0.188841$

$1 \quad-4.629262 \quad 1.913194 \quad 1.413240$

$\begin{array}{llll}6 & -6.565238 & 0.976348 & 1.267918\end{array}$

$\begin{array}{llll}1 & -6.477039 & 0.461958 & 2.229590\end{array}$

$\begin{array}{lllll}1 & -7.149507 & 1.883950 & 1.439484\end{array}$

$1 \quad-7.1298860 .329588 \quad 0.589547$

$6 \quad-4.279759 \quad-0.874189 \quad 1.233566$

$1 \quad-5.271030 \quad-1.2445601 .518372$

$\begin{array}{lllll}1 & -3.779257 & -1.723417 & 0.751139\end{array}$

$\begin{array}{lllll}6 & -3.496424 & -0.454199 & 2.468954\end{array}$

$\begin{array}{llll}-3.409169 & -1.291859 & 3.167315\end{array}$

$\begin{array}{llll}1 & -2.479567 & -0.132931 & 2.215405\end{array}$

$1 \quad-3.987265 \quad 0.366465 \quad 3.003166$

$1 \quad-0.304888-3.329006 \quad 0.579940$

$\begin{array}{lllll}6 & 0.144910 & 2.143618 & -1.155547\end{array}$

$\begin{array}{llll}1 & -0.601485 & 1.752252 & 0.213951\end{array}$

$\begin{array}{llll}8 & 0.730918 & 1.124275 & -1.705397\end{array}$

$1 \quad 0.543531 \quad-3.768038-0.896072$

$\begin{array}{lllll}6 & 1.024413 & 3.254806 & -0.625652\end{array}$

$\begin{array}{lllll}6 & 2.356647 & 3.315220 & -1.035692\end{array}$

$\begin{array}{lllll}6 & 0.533633 & 4.231115 & 0.244100\end{array}$

$\begin{array}{lllll}6 & 3.190027 & 4.328463 & -0.570962\end{array}$

$1 \quad 2.721965 \quad 2.550769 \quad-1.716494$

$\begin{array}{lllll}6 & 1.365798 & 5.240730 & 0.711872\end{array}$

$1 \quad-0.497944 \quad 4.172018 \quad 0.588106$

$\begin{array}{llll}6 & 2.698870 & 5.290380 & 0.306927\end{array}$

$14.228058 \quad 4.363527 \quad-0.891168$

$\begin{array}{lllll}1 & 0.978858 & 5.984618 & 1.403208\end{array}$

$1 \quad 3.351938 \quad 6.075535 \quad 0.678340$

$\begin{array}{llll}6 & -1.114359 & 2.634357 & -1.852699\end{array}$

$1 \quad-1.680085 \quad 1.783041 \quad-2.234048$

$1 \quad-1.752957 \quad 3.256939 \quad-1.220647$

$1 \quad-0.798758 \quad 3.237194 \quad-2.711531$

B-5 I 


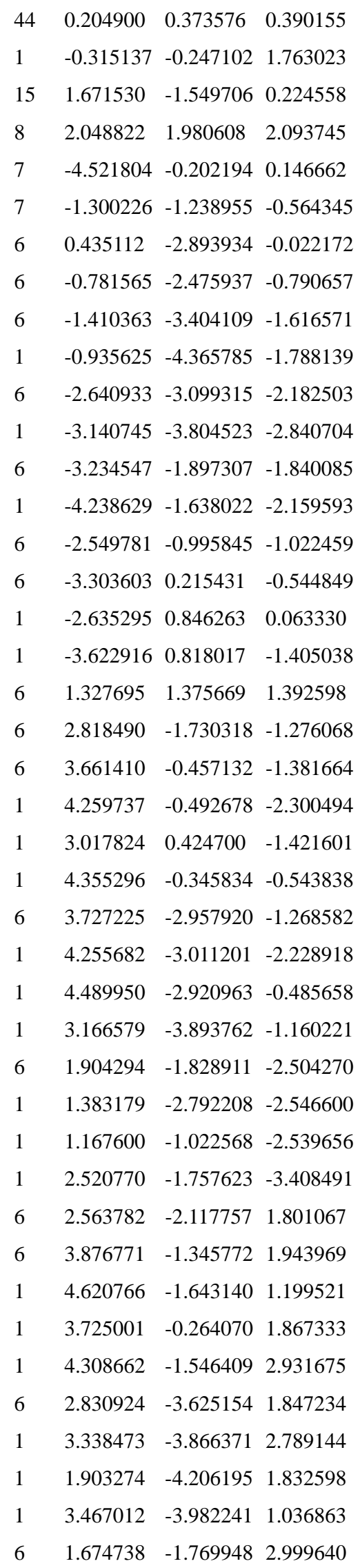

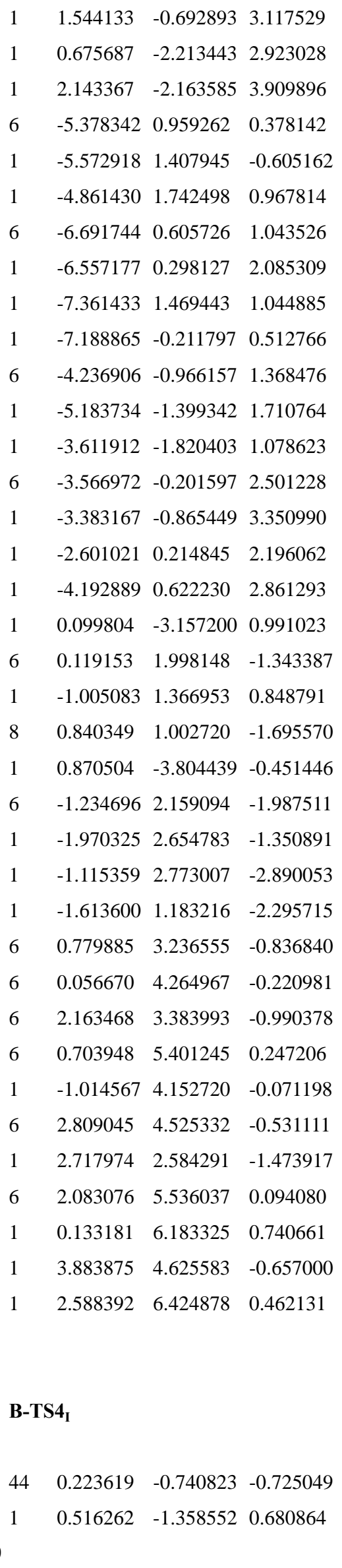



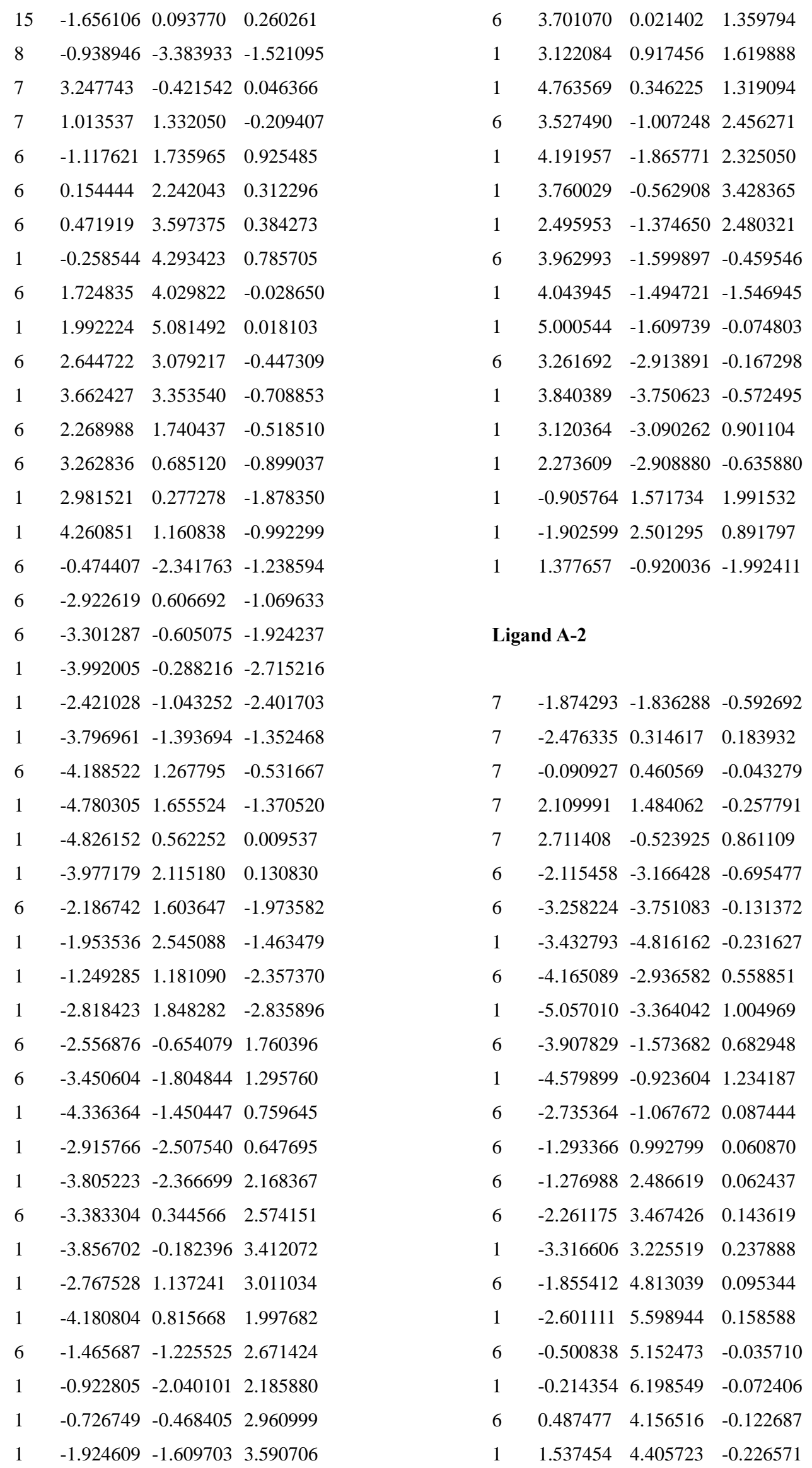

\section{Ligand A-2}

$7 \quad-1.874293-1.836288-0.592692$

$\begin{array}{lllll}7 & -2.476335 & 0.314617 & 0.183932\end{array}$

$\begin{array}{lllll}7 & -0.090927 & 0.460569 & -0.043279\end{array}$

$\begin{array}{llll}7 & 2.109991 & 1.484062 & -0.257791\end{array}$

$\begin{array}{llll}7 & 2.711408 & -0.523925 & 0.861109\end{array}$

$\begin{array}{lllll}6 & -2.115458 & -3.166428 & -0.695477\end{array}$

$6 \quad-3.258224-3.751083-0.131372$

$\begin{array}{lllll}1 & -3.432793 & -4.816162 & -0.231627\end{array}$

$\begin{array}{lllll}6 & -4.165089 & -2.936582 & 0.558851\end{array}$

$\begin{array}{llll}1 & -5.057010 & -3.364042 & 1.004969\end{array}$

$\begin{array}{lllll}6 & -3.907829 & -1.573682 & 0.682948\end{array}$

$\begin{array}{lllll}1 & -4.579899 & -0.923604 & 1.234187\end{array}$

$\begin{array}{lllll}6 & -2.735364 & -1.067672 & 0.087444\end{array}$

$\begin{array}{llll}6 & -1.293366 & 0.992799 & 0.060870\end{array}$

$\begin{array}{llll}6 & -1.276988 & 2.486619 & 0.062437\end{array}$

$\begin{array}{llll}6 & -2.261175 & 3.467426 & 0.143619\end{array}$

$\begin{array}{lllll}1 & -3.316606 & 3.225519 & 0.237888\end{array}$

$\begin{array}{llll}6 & -1.855412 & 4.813039 & 0.095344\end{array}$

$\begin{array}{llll}1 & -2.601111 & 5.598944 & 0.158588\end{array}$

$\begin{array}{llll}6 & -0.500838 & 5.152473 & -0.035710\end{array}$

$\begin{array}{lllll}1 & -0.214354 & 6.198549 & -0.072406\end{array}$

$\begin{array}{lllll}6 & 0.487477 & 4.156516 & -0.122687\end{array}$

$\begin{array}{lllll}1 & 1.537454 & 4.405723 & -0.226571\end{array}$ 


$\begin{array}{llll}6 & 0.079963 & 2.830507 & -0.074368 \\ 6 & 0.832007 & 1.547568 & -0.125876 \\ 6 & 2.867336 & 0.304360 & -0.196460 \\ 6 & 3.830475 & 0.077389 & -1.198793 \\ 1 & 3.938259 & 0.790029 & -2.007111 \\ 6 & 4.612262 & -1.071684 & -1.115195 \\ 1 & 5.351858 & -1.286577 & -1.880093 \\ 6 & 4.444851 & -1.941154 & -0.029394 \\ 1 & 5.047570 & -2.837998 & 0.063869 \\ 6 & 3.491953 & -1.627614 & 0.951054 \\ 6 & -1.091326 & -3.963859 & -1.457797 \\ 1 & -1.036792 & -3.620621 & -2.497404 \\ 1 & -1.324986 & -5.032394 & -1.453238 \\ 1 & -0.097932 & -3.812543 & -1.022474 \\ 6 & 3.276075 & -2.492875 & 2.166528 \\ 1 & 2.216065 & -2.749973 & 2.263470 \\ 1 & 3.558142 & -1.951735 & 3.077862 \\ 1 & 3.862958 & -3.415114 & 2.115714 \\ 1 & -3.277701 & 0.882377 & 0.419635\end{array}$

$$
\begin{array}{llll}
\text { L-1-a } & & & \\
15 & -2.496281 & -0.432296 & -0.185409 \\
7 & -0.053735 & 1.402254 & -0.512482 \\
6 & -2.416303 & 1.361977 & -0.028224 \\
6 & -1.251275 & 2.050944 & -0.209313 \\
6 & -1.118962 & 3.491132 & -0.092411 \\
1 & -2.014830 & 4.053116 & 0.146243 \\
6 & 0.074833 & 4.106363 & -0.268070 \\
1 & 0.140470 & 5.186078 & -0.171535 \\
6 & 1.259640 & 3.361297 & -0.580464 \\
1 & 2.215834 & 3.847389 & -0.724662 \\
6 & 1.154429 & 2.009483 & -0.697217 \\
6 & 2.303819 & 1.110683 & -1.062694 \\
1 & 2.189012 & 0.828687 & -2.116939 \\
1 & 3.240768 & 1.671129 & -0.982558 \\
6 & -4.112935 & -0.691604 & -1.137970 \\
6 & -4.201117 & -2.163405 & -1.566724 \\
1 & -5.074867 & -2.306073 & -2.214995 \\
1 & -3.309664 & -2.461299 & -2.127053 \\
1 & -4.307616 & -2.840603 & -0.715990 \\
6 & -5.393183 & -0.292820 & -0.401867
\end{array}
$$

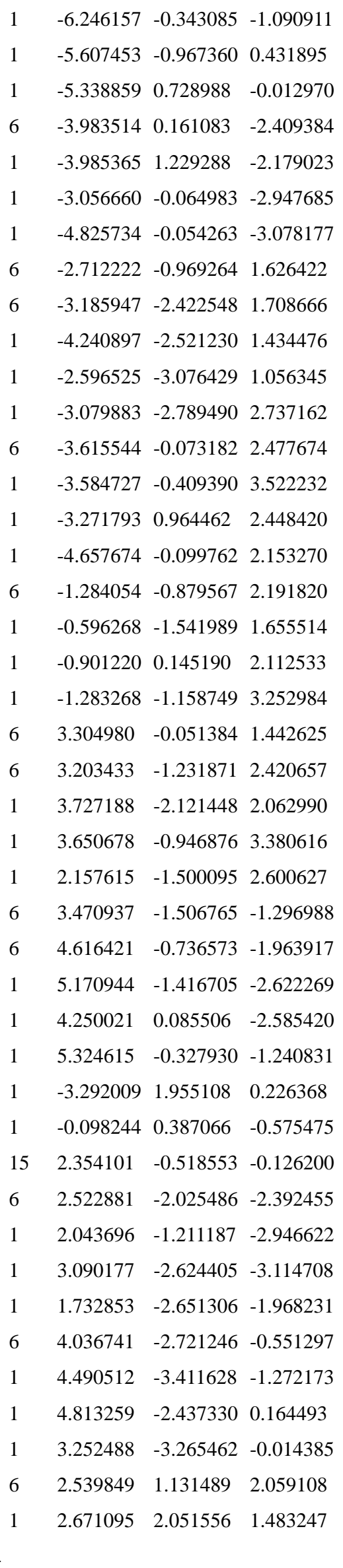




$\begin{array}{llll}1 & 1.465210 & 0.927759 & 2.130420 \\ 1 & 2.916613 & 1.311890 & 3.072720 \\ 6 & 4.769114 & 0.353119 & 1.263425 \\ 1 & 5.166081 & 0.713242 & 2.220690 \\ 1 & 5.393754 & -0.485880 & 0.945433 \\ 1 & 4.883566 & 1.164333 & 0.537046 \\ 0 & 0.017280 & 2.736735 & -0.382067\end{array}$

\section{L-1-b}

$15 \quad-2.427732-0.297812 \quad 0.245834$

$\begin{array}{llll}7 & -0.009312 & 1.105055 & -1.212738\end{array}$

$6 \quad-2.423636 \quad 1.050912 \quad-1.073309$

$\begin{array}{lllll}6 & -1.127704 & 1.818991 & -1.072293\end{array}$

$6 \quad-1.104141 \quad 3.206458 \quad-0.898971$

$\begin{array}{lllll}1 & -2.030643 & 3.756927 & -0.772265\end{array}$

$\begin{array}{lllll}6 & 0.123990 & 3.855932 & -0.900956\end{array}$

$\begin{array}{lllll}1 & 0.176371 & 4.933850 & -0.780896\end{array}$

$\begin{array}{lllll}6 & 1.285946 & 3.108259 & -1.050042\end{array}$

$1 \quad 2.262433 \quad 3.581696 \quad-1.050611$

$\begin{array}{lllll}6 & 1.175302 & 1.721574 & -1.185128\end{array}$

$\begin{array}{lllll}6 & 2.384713 & 0.829653 & -1.275903\end{array}$

$\begin{array}{lllll}1 & 2.346375 & 0.309536 & -2.237887\end{array}$

$\begin{array}{llll}1 & 3.307224 & 1.419217 & -1.243615\end{array}$

$6 \quad-2.426322 \quad-1.891387 \quad-0.787551$

$\begin{array}{lllll}6 & -2.170216 & -3.056815 & 0.181254\end{array}$

$1 \quad-2.080242 \quad-3.990178 \quad-0.387849$

$\begin{array}{lllll}1 & -1.235942 & -2.902553 & 0.729775\end{array}$

$\begin{array}{lllll}1 & -2.975927 & -3.188867 & 0.907354\end{array}$

$6 \quad-3.683761-2.168112-1.612264$

$\begin{array}{lllll}1 & -3.503724 & -3.022002 & -2.277773\end{array}$

$1 \quad-4.539062 \quad-2.423302 \quad-0.980541$

$\begin{array}{lllll}1 & -3.960524 & -1.313282 & -2.238867\end{array}$

$\begin{array}{lllll}6 & -1.214347 & -1.818182 & -1.730428\end{array}$

$1 \quad-1.343292-1.073422-2.521029$

$1 \quad-0.296374-1.566583 \quad-1.191740$

$\begin{array}{lllll}1 & -1.089514 & -2.796907 & -2.210589\end{array}$

$\begin{array}{lllll}6 & -4.168525 & -0.020770 & 0.968596\end{array}$

$6 \quad-4.529083-1.202955 \quad 1.876632$

$1 \quad-4.796115 \quad-2.091343 \quad 1.296946$

$\begin{array}{lllll}1 & -3.702972 & -1.462941 & 2.547189\end{array}$

$\begin{array}{llll}1 & -5.396623 & -0.940961 & 2.494655\end{array}$ $\begin{array}{llll}6 & -5.330474 & 0.231551 & -0.000440\end{array}$

$1 \quad-6.228600 \quad 0.485498 \quad 0.577057$

$1 \quad-5.139296 \quad 1.068246 \quad-0.677987$

$1 \quad-5.568568 \quad-0.643922-0.605168$

$\begin{array}{llll}6 & -3.996831 & 1.224961 & 1.856015\end{array}$

$1 \quad-3.236514 \quad 1.061765 \quad 2.625186$

$\begin{array}{llll}1 & -3.700248 & 2.105791 & 1.275204\end{array}$

$1 \quad-4.946499 \quad 1.460036 \quad 2.351838$

$\begin{array}{llll}6 & 2.515407 & 0.454065 & 1.639681\end{array}$

$\begin{array}{lllll}6 & 3.014435 & -0.486738 & 2.744561\end{array}$

$\begin{array}{lllll}1 & 4.069800 & -0.746227 & 2.624158\end{array}$

$1 \quad 2.9068890 .009236 \quad 3.716644$

$1 \quad 2.431208 \quad-1.4134592 .777504$

$6 \quad 4.022810 \quad-1.374083-0.359133$

$\begin{array}{lllll}6 & 5.296359 & -0.578377 & -0.064843\end{array}$

$1 \quad 6.168788 \quad-1.133139 \quad-0.433838$

$1 \quad 5.292682 \quad 0.396531 \quad-0.563386$

$1 \quad 5.442919-0.415306 \quad 1.005942$

$1 \quad-2.592990 \quad 0.609522 \quad-2.061725$

$1 \quad-3.248806 \quad 1.741238 \quad-0.886431$

$15 \quad 2.354442 \quad-0.5342310 .021256$

$6 \quad 4.001416 \quad-1.715223 \quad-1.859543$

$1 \quad 4.177806 \quad-0.833745-2.482405$

$1 \quad 4.798717 \quad-2.435652 \quad-2.076991$

$1 \quad 3.049504 \quad-2.168427-2.159900$

$\begin{array}{lllll}6 & 4.053557 & -2.706678 & 0.405940\end{array}$

$1 \quad 4.955736 \quad-3.266750 \quad 0.130605$

$1 \quad 4.066681 \quad-2.569134 \quad 1.488735$

$1 \quad 3.182696 \quad-3.319576 \quad 0.154514$

$\begin{array}{lllll}6 & 1.084236 & 0.885583 & 2.015617\end{array}$

$\begin{array}{llll}1 & 0.683335 & 1.638452 & 1.331396\end{array}$

$\begin{array}{lllll}1 & 0.390578 & 0.039815 & 2.014979\end{array}$

$\begin{array}{llll}1 & 1.098491 & 1.326043 & 3.020853\end{array}$

$\begin{array}{llll}6 & 3.401894 & 1.702165 & 1.592522\end{array}$

$1 \quad 3.402760 \quad 2.180846 \quad 2.580223$

$\begin{array}{llll}1 & 4.438340 & 1.477028 & 1.333402\end{array}$

$1 \quad 3.017523 \quad 2.436956 \quad 0.880060$

$\begin{array}{llll}0 & 0.067877 & 2.475371 & -1.053033\end{array}$

\section{L-2-a}

$15 \quad-2.475338-0.505134-0.242664$ 

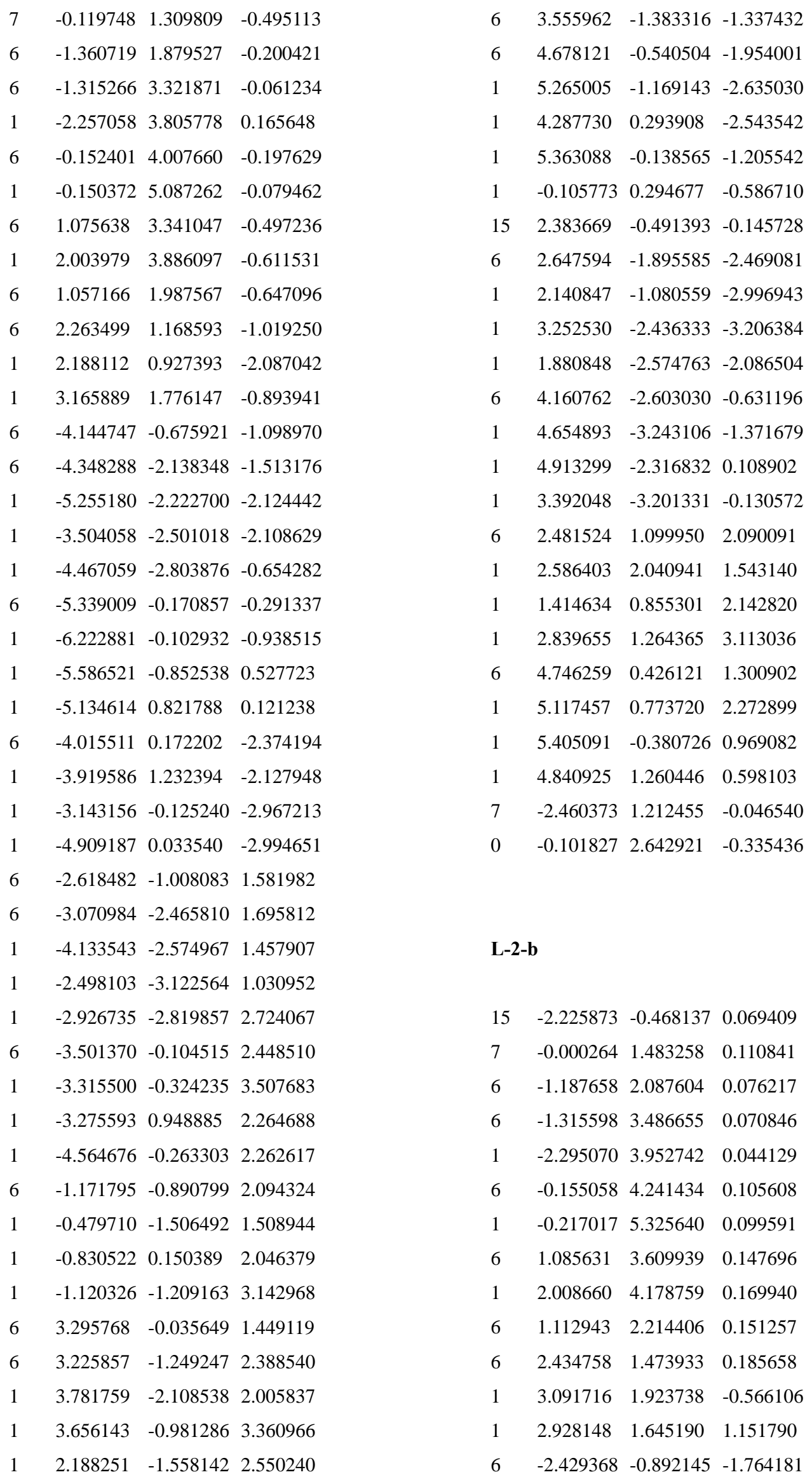

\section{L-2-b}

$\begin{array}{llll}15 & -2.225873 & -0.468137 & 0.069409\end{array}$

$\begin{array}{llll}7 & -0.000264 & 1.483258 & 0.110841\end{array}$

$\begin{array}{llll}6 & -1.187658 & 2.087604 & 0.076217\end{array}$

$\begin{array}{llll}6 & -1.315598 & 3.486655 & 0.070846\end{array}$

$\begin{array}{llll}1 & -2.295070 & 3.952742 & 0.044129\end{array}$

$\begin{array}{llll}6 & -0.155058 & 4.241434 & 0.105608\end{array}$

$\begin{array}{llll}1 & -0.217017 & 5.325640 & 0.099591\end{array}$

$\begin{array}{llll}6 & 1.085631 & 3.609939 & 0.147696\end{array}$

$\begin{array}{llll}1 & 2.008660 & 4.178759 & 0.169940\end{array}$

$\begin{array}{lllll}6 & 1.112943 & 2.214406 & 0.151257\end{array}$

$\begin{array}{llll}6 & 2.434758 & 1.473933 & 0.185658\end{array}$

$1 \quad 3.091716 \quad 1.923738 \quad-0.566106$

$\begin{array}{llll}1 & 2.928148 & 1.645190 & 1.151790\end{array}$

$6 \quad-2.429368-0.892145-1.764181$ 


$$
\begin{array}{llll}
1 & 3.514016 & -2.692695 & -1.382123 \\
6 & 1.139485 & -0.408448 & 2.334575 \\
1 & 1.363033 & 0.645649 & 2.529830 \\
1 & 0.192931 & -0.459481 & 1.791031 \\
1 & 1.017716 & -0.906203 & 3.305050 \\
6 & 3.556519 & -0.991156 & 2.383940 \\
1 & 3.370302 & -1.358119 & 3.401427 \\
1 & 4.371087 & -1.588785 & 1.966389 \\
1 & 3.898187 & 0.046201 & 2.467946 \\
7 & -2.305012 & 1.268947 & 0.041248 \\
1 & -3.173026 & 1.736833 & -0.184077 \\
0 & -0.084417 & 2.826612 & 0.108952
\end{array}
$$

\section{L-3-a}

$\begin{array}{llll}15 & -1.733683 & -0.397544 & -0.062436\end{array}$

$\begin{array}{llll}7 & 1.160201 & 0.729455 & 0.002878\end{array}$

$\begin{array}{llll}6 & 0.132913 & 1.665963 & 0.050988\end{array}$

$\begin{array}{llll}6 & 0.599753 & 3.040032 & 0.116756\end{array}$

$\begin{array}{llll}6 & 1.919256 & 3.341207 & 0.128397\end{array}$

$\begin{array}{llll}6 & 2.916132 & 2.312479 & 0.077346\end{array}$

$\begin{array}{llll}6 & 2.492620 & 1.019242 & 0.014967\end{array}$

$6 \quad-2.932676-0.354644-1.534645$

$6 \quad-3.314344-1.794425-1.905442$

$\begin{array}{lllll}6 & -4.195315 & 0.485201 & -1.339758\end{array}$

$\begin{array}{lllll}6 & -2.122529 & 0.232090 & -2.700091\end{array}$

$\begin{array}{llll}6 & -2.713298 & -0.581185 & 1.556872\end{array}$

$\begin{array}{llll}6 & -3.642077 & -1.797025 & 1.494337\end{array}$

$\begin{array}{llll}6 & -3.502955 & 0.655724 & 1.994766\end{array}$

$\begin{array}{lllll}6 & -1.624865 & -0.846708 & 2.609384\end{array}$

$\begin{array}{llll}1 & -0.155363 & 3.817248 & 0.157214\end{array}$

$\begin{array}{llll}1 & 2.234852 & 4.378717 & 0.177418\end{array}$

$\begin{array}{llll}1 & 3.967172 & 2.562641 & 0.092344\end{array}$

$1 \quad-3.907214-1.792496-2.828648$

$1 \quad-2.420656-2.402369-2.077249$

$1 \quad-3.910761 \quad-2.281293-1.130759$

$1 \quad-4.749818 \quad 0.545881 \quad-2.285439$

$1 \quad-4.866222 \quad 0.047115 \quad-0.595372$

$1 \quad-3.956008 \quad 1.508016 \quad-1.030650$

$1 \quad-1.881126 \quad 1.283987 \quad-2.529145$

$1 \quad-1.181141 \quad-0.309654 \quad-2.844385$

$\begin{array}{llll}1 & -2.705541 & 0.152924 & -3.625808\end{array}$ 


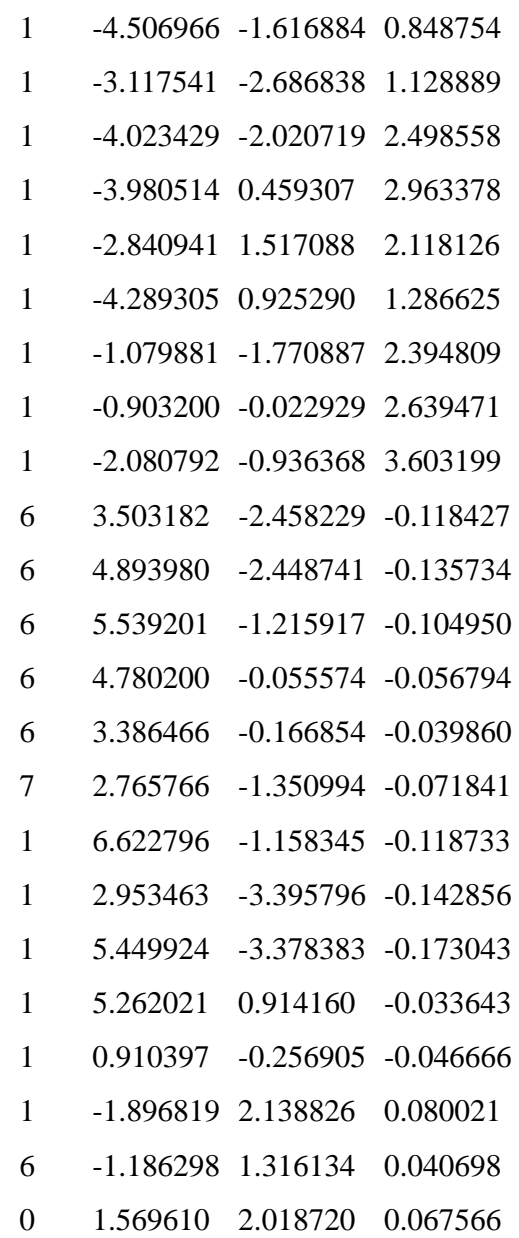

\section{L-3-b}

$\begin{array}{llll}15 & 1.901553 & -0.175048 & 0.433263\end{array}$

$\begin{array}{llll}7 & -1.187325 & 0.501431 & -0.531397\end{array}$

$\begin{array}{llll}6 & -0.145901 & 1.299895 & -0.775706\end{array}$

$\begin{array}{llll}6 & -0.239286 & 2.692559 & -0.700939\end{array}$

$\begin{array}{llll}6 & -1.461545 & 3.259624 & -0.364007\end{array}$

$\begin{array}{lllll}6 & -2.546202 & 2.429005 & -0.111485\end{array}$

$\begin{array}{lllll}6 & -2.360033 & 1.046375 & -0.206258\end{array}$

$\begin{array}{llll}6 & 3.571881 & 0.726350 & 0.508959\end{array}$

$\begin{array}{llll}6 & 4.238442 & 0.365719 & 1.845602\end{array}$

$\begin{array}{lllll}6 & 4.550852 & 0.474246 & -0.638257\end{array}$

$\begin{array}{llll}6 & 3.235001 & 2.226346 & 0.559373\end{array}$

$6 \quad 2.152783 \quad-1.960043 \quad-0.161956$

$\begin{array}{lllll}6 & 3.086008 & -2.687626 & 0.813264\end{array}$

$6 \quad 2.667276 \quad-2.176786-1.589778$

$\begin{array}{lllll}6 & 0.746283 & -2.577184 & -0.044255\end{array}$

$\begin{array}{llll}1 & 0.627101 & 3.310555 & -0.913005\end{array}$
$1 \quad-1.572232 \quad 4.338352 \quad-0.311260$

$\begin{array}{llll}1 & -3.521972 & 2.840405 & 0.121969\end{array}$

$\begin{array}{llll}1 & 5.152465 & 0.959291 & 1.971361\end{array}$

$\begin{array}{llll}1 & 3.572906 & 0.582933 & 2.686533\end{array}$

$\begin{array}{llll}1 & 4.519011 & -0.688401 & 1.899288\end{array}$

$\begin{array}{lllll}1 & 5.397540 & 1.168822 & -0.563501\end{array}$

$1 \quad 4.958556-0.539962-0.604633$

$1 \quad 4.083626 \quad 0.624151 \quad-1.617474$

$1 \quad 2.903518 \quad 2.607110 \quad-0.410891$

$\begin{array}{llll}1 & 2.454048 & 2.440758 & 1.297543\end{array}$

$\begin{array}{llll}1 & 4.131826 & 2.790599 & 0.841057\end{array}$

$\begin{array}{llll}1 & 4.132772 & -2.409335 & 0.655711\end{array}$

$\begin{array}{llll}1 & 2.824784 & -2.481057 & 1.856774\end{array}$

$\begin{array}{lllll}1 & 3.007088 & -3.769999 & 0.654741\end{array}$

$1 \quad 2.745794 \quad-3.255300 \quad-1.776487$

$\begin{array}{lllll}1 & 1.987618 & -1.775569 & -2.345780\end{array}$

$1 \quad 3.655002-1.742776-1.753306$

$\begin{array}{lllll}1 & 0.411565 & -2.585890 & 0.997287\end{array}$

$1 \quad-0.005389-2.025873-0.620503$

$1 \quad 0.771131 \quad-3.612109-0.406886$

$6 \quad-4.483277-1.891323-0.479305$

$\begin{array}{lllll}6 & -5.526669 & -1.680756 & 0.420011\end{array}$

$\begin{array}{llll}6 & -5.521495 & -0.506504 & 1.162676\end{array}$

$\begin{array}{llll}6 & -4.486271 & 0.400244 & 0.971489\end{array}$

$\begin{array}{llll}6 & -3.488674 & 0.099706 & 0.038392\end{array}$

$7 \quad-3.485202-1.033282-0.672758$

$\begin{array}{llll}1 & -6.304282 & -0.303459 & 1.886762\end{array}$

$1 \quad-4.451853-2.799768-1.077225$

$\begin{array}{llll}1 & -6.311339 & -2.420105 & 0.534342\end{array}$

$\begin{array}{llll}1 & -4.433899 & 1.314423 & 1.552859\end{array}$

$\begin{array}{lllll}6 & 1.133587 & 0.587228 & -1.114433\end{array}$

$\begin{array}{lllll}1 & 0.874125 & -0.225808 & -1.794348\end{array}$

$1 \quad 1.838336 \quad 1.244236 \quad-1.631111$

$\begin{array}{lllll}0 & -1.317406 & 1.855538 & -0.433519\end{array}$

\section{L-4-a}

$15 \quad-1.717362-0.386008-0.329882$

$\begin{array}{lllll}7 & 1.046667 & 0.714730 & -0.006733\end{array}$

$\begin{array}{lllll}6 & -0.034263 & 1.567608 & 0.201109\end{array}$

$\begin{array}{llll}6 & 0.358451 & 2.928399 & 0.524027\end{array}$

$\begin{array}{llll}6 & 1.662997 & 3.285629 & 0.622152\end{array}$ 


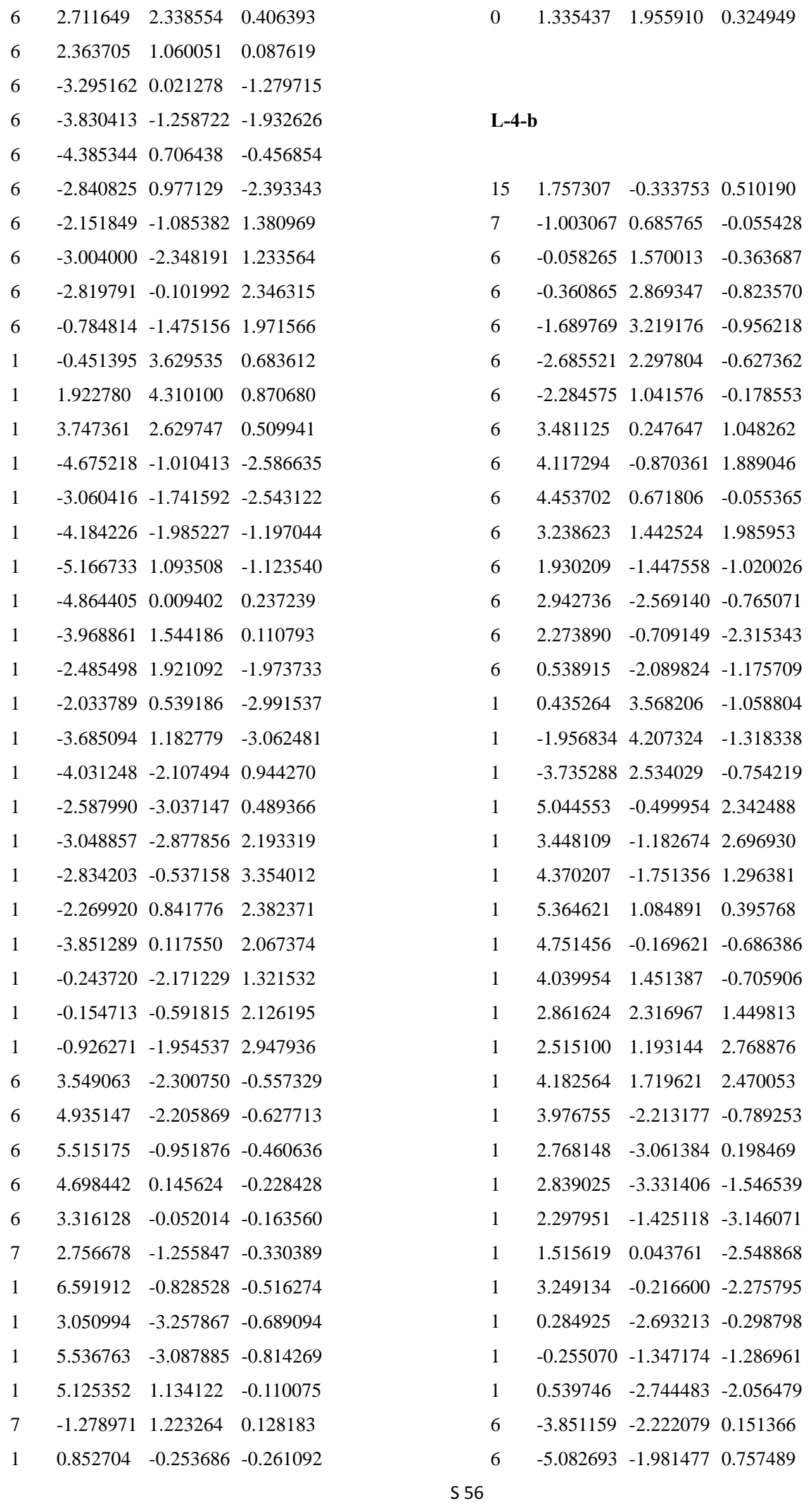




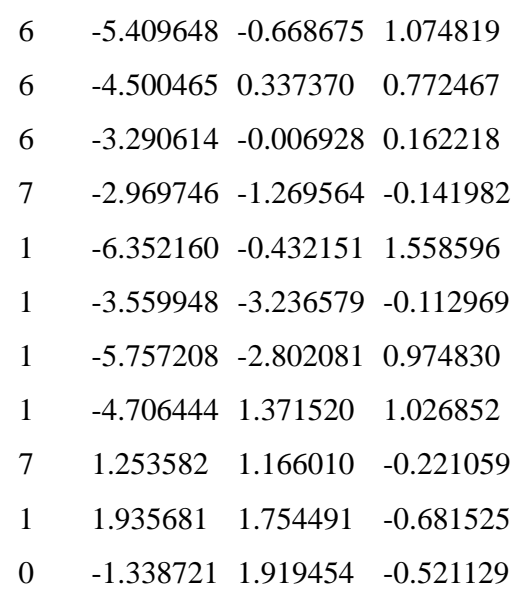

L-5-a

$7 \quad-2.036180 \quad-0.506567 \quad-0.204139$

$\begin{array}{llll}7 & 0.000051 & 1.453526 & -0.000016\end{array}$

$7 \quad 2.036146 \quad-0.506590 \quad 0.204085$

$\begin{array}{lllll}6 & -2.491986 & -1.743802 & -0.403709\end{array}$

$6 \quad-3.834404-2.090656-0.222203$

$1 \quad-4.159000 \quad-3.111516-0.394817$

$\begin{array}{lllll}6 & -4.730532 & -1.103508 & 0.169995\end{array}$

$\begin{array}{llll}1 & -5.779240 & -1.339593 & 0.323475\end{array}$

$\begin{array}{llll}6 & -4.270922 & 0.191507 & 0.345055\end{array}$

$\begin{array}{llll}1 & -4.943330 & 0.994647 & 0.627402\end{array}$

$\begin{array}{llll}6 & -2.907882 & 0.464613 & 0.142769\end{array}$

$\begin{array}{llll}6 & -1.129190 & 2.230896 & 0.128435\end{array}$

$\begin{array}{llll}6 & -0.666623 & 3.619432 & 0.090042\end{array}$

$\begin{array}{lllll}6 & 0.666765 & 3.619422 & -0.090064\end{array}$

$\begin{array}{llll}6 & 1.129302 & 2.230879 & -0.128469\end{array}$

$\begin{array}{llll}6 & 2.907920 & 0.464546 & -0.142761\end{array}$

$\begin{array}{llll}6 & 4.270962 & 0.191382 & -0.344962\end{array}$

$\begin{array}{llll}1 & 4.943421 & 0.994493 & -0.627274\end{array}$

$\begin{array}{lllll}6 & 4.730504 & -1.103650 & -0.169867\end{array}$

$\begin{array}{lllll}1 & 5.779213 & -1.339782 & -0.323272\end{array}$

$\begin{array}{lllll}6 & 3.834304 & -2.090756 & 0.222275\end{array}$

$\begin{array}{lllll}1 & 4.158845 & -3.111628 & 0.394925\end{array}$

$\begin{array}{lllll}6 & 2.491888 & -1.743844 & 0.403684\end{array}$

$\begin{array}{lllll}6 & -1.494737 & -2.774013 & -0.863990\end{array}$

$1 \quad-0.554784-2.282942-1.120093$

$1 \quad-1.870835-3.310344-1.739825$

$\begin{array}{lllll}1 & -1.303003 & -3.515029 & -0.081244\end{array}$

$\begin{array}{lllll}6 & 1.494567 & -2.774017 & 0.863888\end{array}$

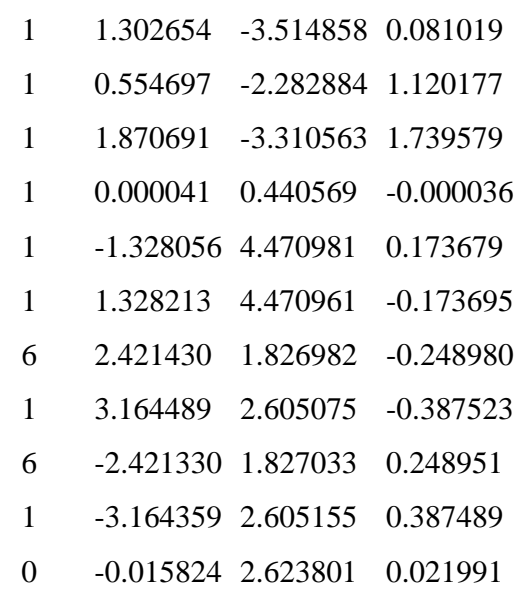

\section{L-5-b}

$\begin{array}{llll}7 & -3.934439 & 0.149824 & 0.444509\end{array}$

$\begin{array}{llll}7 & 0.360687 & 1.201168 & 0.574323\end{array}$

$\begin{array}{lllll}7 & 2.497541 & -0.673028 & -0.117042\end{array}$

$\begin{array}{llll}6 & -4.565612 & -0.832908 & -0.214163\end{array}$

$6 \quad-3.880679-1.907479-0.777775$

$1 \quad-4.425977-2.684824-1.302855$

$6 \quad-2.494546-1.956893 \quad-0.657078$

$1 \quad-1.934914-2.780190-1.091456$

$\begin{array}{lllll}6 & -1.835931 & -0.941965 & 0.020690\end{array}$

$\begin{array}{lllll}1 & -0.755168 & -0.922670 & 0.128835\end{array}$

$\begin{array}{lllll}6 & -2.607989 & 0.093029 & 0.559942\end{array}$

$\begin{array}{llll}6 & -0.784276 & 1.807468 & 0.617499\end{array}$

$\begin{array}{lllll}6 & -0.799219 & 3.066856 & -0.154489\end{array}$

$\begin{array}{lllll}6 & 0.438003 & 3.195353 & -0.669206\end{array}$

$\begin{array}{lllll}6 & 1.178995 & 1.996079 & -0.246009\end{array}$

$\begin{array}{lllll}6 & 3.180766 & 0.459928 & -0.325272\end{array}$

$\begin{array}{lllll}6 & 4.580500 & 0.512491 & -0.290781\end{array}$

$\begin{array}{lllll}1 & 5.094676 & 1.448084 & -0.484788\end{array}$

$\begin{array}{lllll}6 & 5.282323 & -0.646402 & 0.010187\end{array}$

$\begin{array}{lllll}1 & 6.366779 & -0.639545 & 0.055711\end{array}$

$\begin{array}{lllll}6 & 4.571196 & -1.815198 & 0.249425\end{array}$

$\begin{array}{lllll}1 & 5.081958 & -2.743079 & 0.484211\end{array}$

$\begin{array}{lllll}6 & 3.174222 & -1.785658 & 0.167864\end{array}$

$6 \quad-6.061521-0.702756-0.318002$

$\begin{array}{lllll}1 & -6.497456 & -0.583639 & 0.676918\end{array}$

$\begin{array}{lllll}1 & -6.509519 & -1.573652 & -0.800593\end{array}$

$\begin{array}{lllll}1 & -6.321777 & 0.189480 & -0.894323\end{array}$

$\begin{array}{llll}6 & 2.344630 & -3.022004 & 0.387696\end{array}$ 
$\begin{array}{llll}1 & 1.788824 & -3.263557 & -0.522545 \\ 1 & 1.611648 & -2.839613 & 1.177423 \\ 1 & 2.959834 & -3.880848 & 0.662676 \\ 1 & -1.647229 & 3.731119 & -0.256738 \\ 1 & 0.832547 & 3.981561 & -1.299349 \\ 6 & 2.437301 & 1.692068 & -0.623251 \\ 1 & 2.974430 & 2.441218 & -1.202119 \\ 6 & -1.953810 & 1.218577 & 1.347015 \\ 1 & -2.717640 & 1.971675 & 1.549528 \\ 1 & -1.593650 & 0.819219 & 2.301184 \\ 0 & 0.085806 & 2.220452 & 0.018565\end{array}$

\section{L-6-a}

$7 \quad-2.024843 \quad-0.512705 \quad-0.221526$

$\begin{array}{llll}7 & -2.378878 & 1.824330 & 0.134810\end{array}$

$\begin{array}{llll}7 & 0.000027 & 1.344551 & -0.000102\end{array}$

$\begin{array}{llll}7 & 2.378966 & 1.824181 & -0.134989\end{array}$

$\begin{array}{llll}7 & 2.024697 & -0.512914 & 0.220895\end{array}$

$\begin{array}{lllll}6 & -2.545863 & -1.737750 & -0.341141\end{array}$

$6 \quad-3.901545-1.998203-0.129003$

$\begin{array}{lllll}1 & -4.280977 & -3.008805 & -0.237374\end{array}$

$\begin{array}{llll}6 & -4.742170 & -0.943912 & 0.211764\end{array}$

$\begin{array}{llll}1 & -5.800211 & -1.115523 & 0.383778\end{array}$

$\begin{array}{llll}6 & -4.212343 & 0.332062 & 0.307342\end{array}$

$1 \quad-4.814955 \quad 1.200678 \quad 0.542281$

$\begin{array}{lllll}6 & -2.840849 & 0.510505 & 0.078924\end{array}$

$\begin{array}{llll}6 & -1.134704 & 2.131821 & 0.063194\end{array}$

$\begin{array}{lllll}6 & -0.666802 & 3.535187 & 0.044901\end{array}$

$\begin{array}{lllll}6 & 0.666978 & 3.535141 & -0.045432\end{array}$

$\begin{array}{llll}6 & 1.134797 & 2.131744 & -0.063521\end{array}$

$\begin{array}{lllll}6 & 2.840900 & 0.510358 & -0.078803\end{array}$

$\begin{array}{lllll}6 & 4.212588 & 0.331983 & -0.306155\end{array}$

$\begin{array}{llll}1 & 4.815330 & 1.200644 & -0.540593\end{array}$

$\begin{array}{lllll}6 & 4.742412 & -0.943954 & -0.210178\end{array}$

$1 \quad 5.800605 \quad-1.115495 \quad-0.381325$

$\begin{array}{lllll}6 & 3.901559 & -1.998310 & 0.129845\end{array}$

$\begin{array}{lllll}1 & 4.280958 & -3.008895 & 0.238468\end{array}$

$\begin{array}{lllll}6 & 2.545685 & -1.737952 & 0.340827\end{array}$

$\begin{array}{lllll}6 & -1.602208 & -2.846854 & -0.722397\end{array}$

$\begin{array}{llll}1 & -0.680740 & -2.421948 & -1.123178\end{array}$

$\begin{array}{llll}1 & -2.052896 & -3.504949 & -1.469828\end{array}$
$1 \quad-1.348752 \quad-3.460346 \quad 0.148475$

$\begin{array}{lllll}6 & 1.601727 & -2.847160 & 0.721012\end{array}$

$11.347991 \quad-3.459603-0.150517$

$1 \quad 0.680388 \quad-2.4224191 .122280$

$1 \quad 2.052253 \quad-3.5062201 .467681$

$\begin{array}{llll}1 & 0.000015 & 0.331853 & -0.000233\end{array}$

$1 \quad-1.356826 \quad 4.365391 \quad 0.089155$

$1 \quad 1.357055 \quad 4.365297 \quad-0.089759$

$\begin{array}{llll}0 & -0.018965 & 2.507828 & 0.024164\end{array}$

\section{L-6-b}

$\begin{array}{llll}7 & -2.065454 & -0.568848 & -0.468394\end{array}$

$\begin{array}{llll}7 & -2.257014 & 1.694528 & 0.093054\end{array}$

$\begin{array}{llll}7 & 0.082420 & 1.288489 & -0.061948\end{array}$

$\begin{array}{lllll}7 & 2.420450 & 1.858854 & -0.127884\end{array}$

$\begin{array}{llll}7 & 2.397537 & -0.280534 & 0.856048\end{array}$

$6 \quad-2.594922-1.796224-0.542776$

$\begin{array}{lllll}6 & -3.906028 & -2.069634 & -0.156707\end{array}$

$1 \quad-4.300983 \quad-3.076143 \quad-0.235601$

$6 \quad-4.689981-1.0236050 .325902$

$1 \quad-5.715555 \quad-1.200326 \quad 0.634645$

$\begin{array}{llll}6 & -4.145974 & 0.244979 & 0.419619\end{array}$

$1 \quad-4.719156 \quad 1.080801 \quad 0.807968$

$\begin{array}{llll}6 & -2.814664 & 0.410424 & 0.007916\end{array}$

$\begin{array}{llll}6 & -0.945263 & 2.066020 & 0.050457\end{array}$

$\begin{array}{llll}6 & -0.607587 & 3.521727 & 0.147538\end{array}$

$\begin{array}{lllll}6 & 0.722945 & 3.575677 & 0.067200\end{array}$

$\begin{array}{lllll}6 & 1.184617 & 2.159512 & -0.044682\end{array}$

$\begin{array}{lllll}6 & 2.804507 & 0.505777 & -0.138035\end{array}$

$6 \quad 3.663376 \quad 0.078189-1.158680$

$1 \quad 3.985559 \quad 0.781801 \quad-1.916911$

$\begin{array}{lllll}6 & 4.059752 & -1.248322 & -1.157688\end{array}$

$14.711755-1.627058-1.938959$

$6 \quad 3.621331-2.086440-0.135310$

$1 \quad 3.918610 \quad-3.128985-0.098416$

$\begin{array}{lllll}6 & 2.800865 & -1.555787 & 0.860010\end{array}$

$\begin{array}{lllll}6 & -1.684223 & -2.862445 & -1.085683\end{array}$

$1 \quad-1.450340 \quad-2.652761 \quad-2.133071$

$1 \quad-2.135900-3.853770-1.015877$

$1 \quad-0.739131-2.852042-0.537707$

$\begin{array}{llll}6 & 2.313558 & -2.392574 & 2.012534\end{array}$ 


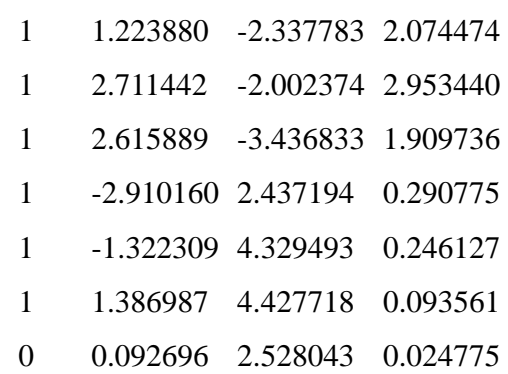

$$
\begin{aligned}
& \text { L-7-a } \\
& 7 \quad 1.340344 \quad-1.986902-0.236475 \\
& \begin{array}{lllll}
7 & -0.651025 & 0.000003 & 0.000020
\end{array} \\
& \begin{array}{llll}
7 & 1.340097 & 1.987033 & 0.236588
\end{array} \\
& \begin{array}{lllll}
6 & 2.583059 & -2.416682 & -0.460149
\end{array} \\
& 6 \quad 2.964182 \quad-3.748476 \quad-0.270675 \\
& 1 \quad 3.988176 \quad-4.051848-0.462442 \\
& \begin{array}{lllll}
6 & 2.006230 & -4.661448 & 0.152953
\end{array} \\
& \begin{array}{lllll}
1 & 2.269241 & -5.702680 & 0.313587
\end{array} \\
& \begin{array}{lllll}
6 & 0.704641 & -4.229610 & 0.349952
\end{array} \\
& \begin{array}{lllll}
1 & -0.076380 & -4.917479 & 0.656218
\end{array} \\
& \begin{array}{lllll}
6 & 0.396175 & -2.876016 & 0.138947
\end{array} \\
& \begin{array}{lllll}
6 & -1.411480 & -1.142248 & 0.136982
\end{array} \\
& \begin{array}{lllll}
6 & -2.812877 & -0.693234 & 0.099010
\end{array} \\
& \begin{array}{lllll}
6 & -4.006651 & -1.400144 & 0.198054
\end{array} \\
& \begin{array}{llll}
1 & -4.012113 & -2.475139 & 0.349009
\end{array} \\
& \begin{array}{lllll}
6 & -5.200554 & -0.693078 & 0.098843
\end{array} \\
& \begin{array}{lllll}
1 & -6.145546 & -1.221058 & 0.174764
\end{array} \\
& \begin{array}{lllll}
6 & -5.200623 & 0.692630 & -0.098844
\end{array} \\
& \begin{array}{llll}
1 & -6.145668 & 1.220516 & -0.174769
\end{array} \\
& \begin{array}{lllll}
6 & -4.006790 & 1.399813 & -0.198044
\end{array} \\
& \begin{array}{llll}
1 & -4.012357 & 2.474807 & -0.349002
\end{array} \\
& \begin{array}{lllll}
6 & -2.812948 & 0.693021 & -0.098983
\end{array} \\
& \begin{array}{lllll}
6 & -1.411597 & 1.142176 & -0.136949
\end{array} \\
& \begin{array}{lllll}
6 & 0.395905 & 2.876071 & -0.138952
\end{array} \\
& \begin{array}{lllll}
6 & 0.704322 & 4.229649 & -0.350122
\end{array} \\
& 1 \quad-0.0767194 .917453 \quad-0.656486 \\
& \begin{array}{lllll}
6 & 2.005899 & 4.661552 & -0.153183
\end{array} \\
& \begin{array}{llll}
1 & 2.268878 & 5.702770 & -0.313953
\end{array} \\
& \begin{array}{llll}
6 & 2.963881 & 3.748662 & 0.270551
\end{array} \\
& \begin{array}{llll}
1 & 3.987866 & 4.052088 & 0.462281
\end{array} \\
& \begin{array}{llll}
6 & 2.582798 & 2.416880 & 0.460204
\end{array} \\
& \begin{array}{lllll}
6 & 3.577866 & -1.404260 & -0.964089
\end{array}
\end{aligned}
$$

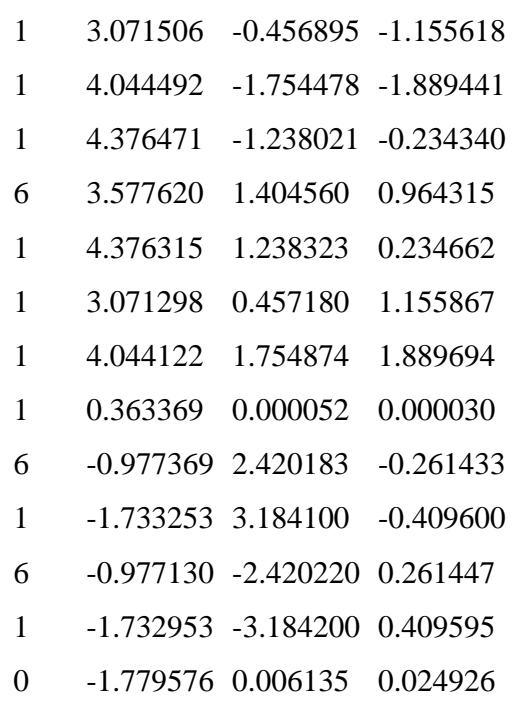

$$
\text { L-7-b }
$$

$\begin{array}{llll}7 & 3.780351 & 0.946513 & 0.714170\end{array}$

$\begin{array}{llll}7 & -0.649788 & 0.063384 & 0.688189\end{array}$

$\begin{array}{llll}7 & -3.401304 & 0.903763 & -0.029298\end{array}$

$\begin{array}{llll}6 & 4.711282 & 1.335459 & -0.168276\end{array}$

$\begin{array}{lllll}6 & 4.408897 & 2.161964 & -1.249651\end{array}$

$1 \quad 5.190542 \quad 2.459003 \quad-1.940836$

$\begin{array}{lllll}6 & 3.095439 & 2.587393 & -1.425142\end{array}$

$\begin{array}{llll}1 & 2.830659 & 3.224512 & -2.263348\end{array}$

$\begin{array}{lllll}6 & 2.130840 & 2.181317 & -0.515498\end{array}$

$1 \quad 1.089378 \quad 2.471611 \quad-0.612657$

$\begin{array}{lllll}6 & 2.524987 & 1.363402 & 0.546975\end{array}$

$\begin{array}{lllll}6 & 0.605496 & -0.148160 & 0.918365\end{array}$

$\begin{array}{lllll}6 & 1.064596 & -1.476290 & 0.469345\end{array}$

$\begin{array}{lllll}6 & 2.298352 & -2.113545 & 0.506992\end{array}$

$\begin{array}{lllll}1 & 3.173375 & -1.611148 & 0.910358\end{array}$

$\begin{array}{lllll}6 & 2.369154 & -3.408484 & -0.006672\end{array}$

$\begin{array}{llll}1 & 3.314633 & -3.941162 & 0.002576\end{array}$

$\begin{array}{lllll}6 & 1.236638 & -4.032539 & -0.539046\end{array}$

$\begin{array}{lllll}1 & 1.321799 & -5.040158 & -0.933671\end{array}$

$\begin{array}{lllll}6 & 0.002023 & -3.383666 & -0.576014\end{array}$

$1 \quad-0.867487-3.880757-0.996617$

$\begin{array}{lllll}6 & -0.072341 & -2.095188 & -0.062983\end{array}$

$\begin{array}{llll}6 & -1.162240 & -1.102978 & 0.073152\end{array}$

$6 \quad-3.593346 \quad-0.406968-0.226719$

$6 \quad-4.872986-0.963719-0.367765$

$1 \quad-4.986846 \quad-2.029306 \quad-0.537991$ 


$\begin{array}{llll}6 & -5.978617 & -0.131139 & -0.271806 \\ 1 & -6.982244 & -0.533478 & -0.368159 \\ 6 & -5.776735 & 1.224557 & -0.049373 \\ 1 & -6.613172 & 1.910739 & 0.032720 \\ 6 & -4.464782 & 1.701570 & 0.059714 \\ 6 & 6.104680 & 0.820707 & 0.071015 \\ 1 & 6.434249 & 1.084923 & 1.078839 \\ 1 & 6.814153 & 1.225991 & -0.652827 \\ 1 & 6.117583 & -0.270726 & 0.000486 \\ 6 & -4.177421 & 3.165020 & 0.265104 \\ 1 & -3.778716 & 3.599821 & -0.656300 \\ 1 & -3.416406 & 3.285572 & 1.038282 \\ 1 & -5.075236 & 3.718160 & 0.548291 \\ 6 & -2.438055 & -1.307703 & -0.307987 \\ 1 & -2.660416 & -2.293553 & -0.713375 \\ 6 & 1.497832 & 0.876477 & 1.549144 \\ 1 & 2.034556 & 0.438224 & 2.395778 \\ 1 & 0.872633 & 1.701187 & 1.899785 \\ 0 & -0.033859 & -0.914399 & 0.424583\end{array}$

$$
\begin{array}{llll}
\text { L-8-a } & & & \\
7 & & & \\
7 & -0.939437 & -2.143356 & 0.339567 \\
7 & 0.602134 & 0.107512 & 0.006291 \\
7 & -1.670215 & 1.732909 & -0.207151 \\
6 & -2.085431 & -2.811539 & 0.503255 \\
6 & -2.226287 & -4.153412 & 0.146321 \\
1 & -3.177881 & -4.654812 & 0.288874 \\
6 & -1.124907 & -4.824017 & -0.377000 \\
1 & -1.201714 & -5.868347 & -0.664228 \\
6 & 0.076987 & -4.149663 & -0.505850 \\
1 & 0.977263 & -4.624846 & -0.875736 \\
6 & 0.135764 & -2.798098 & -0.132639 \\
6 & 1.525499 & -0.908085 & -0.094465 \\
6 & 2.846049 & -0.249125 & -0.055523 \\
6 & 4.115225 & -0.802806 & -0.126910 \\
1 & 4.234783 & -1.875025 & -0.241724 \\
6 & 5.202038 & 0.063625 & -0.046365 \\
1 & 6.211903 & -0.329898 & -0.098949 \\
6 & 5.009159 & 1.442324 & 0.102609 \\
1 & 5.873381 & 2.096062 & 0.162890 \\
6 & 3.730347 & 1.990490 & 0.171710
\end{array}
$$

$$
\begin{array}{llll}
1 & 3.593326 & 3.061586 & 0.284492 \\
6 & 2.645392 & 1.124104 & 0.093156 \\
6 & 1.189854 & 1.359203 & 0.110132 \\
6 & -0.872375 & 2.781521 & 0.080900 \\
6 & -1.383970 & 4.079231 & 0.221816 \\
1 & -0.714910 & 4.900141 & 0.456878 \\
6 & -2.744176 & 4.287027 & 0.047088 \\
1 & -3.166104 & 5.281771 & 0.152899 \\
6 & -3.554764 & 3.208288 & -0.280806 \\
1 & -4.619605 & 3.333179 & -0.447615 \\
6 & -2.972138 & 1.942008 & -0.403205 \\
6 & -3.229721 & -2.059485 & 1.132129 \\
1 & -2.927683 & -1.029595 & 1.328229 \\
1 & -3.518353 & -2.527488 & 2.078315 \\
1 & -4.111408 & -2.057858 & 0.483887 \\
6 & -3.807239 & 0.749754 & -0.788529 \\
1 & -4.517297 & 0.494232 & 0.004372 \\
1 & -3.158863 & -0.108962 & -0.969987 \\
1 & -4.385048 & 0.960961 & -1.692851 \\
1 & -0.400801 & -0.039459 & 0.036125 \\
6 & 0.561829 & 2.552581 & 0.192726 \\
1 & 1.188454 & 3.431595 & 0.304738 \\
7 & 1.378623 & -2.180892 & -0.214982 \\
0 & 1.748065 & 0.293007 & 0.021845
\end{array}
$$

\section{L-8-b}

$\begin{array}{llll}7 & -0.079674 & 2.527493 & 0.524315\end{array}$

$7 \quad-0.473461 \quad-0.259864-0.070035$

$7 \quad 2.363947 \quad-1.025695 \quad-0.269940$

$\begin{array}{llll}6 & 0.721203 & 3.595290 & 0.557324\end{array}$

$\begin{array}{lllll}6 & 0.367902 & 4.816055 & -0.022034\end{array}$

$\begin{array}{llll}1 & 1.045337 & 5.661621 & 0.027938\end{array}$

$6 \quad-0.866093 \quad 4.916730 \quad-0.656046$

$1 \quad-1.1754995 .849778 \quad-1.116893$

$\begin{array}{llll}6 & -1.694415 & 3.807130 & -0.713371\end{array}$

$1 \quad-2.650621 \quad 3.839388 \quad-1.225535$

$\begin{array}{llll}6 & -1.241977 & 2.628214 & -0.106723\end{array}$

$\begin{array}{llll}6 & -1.690842 & 0.183250 & -0.062258\end{array}$

$6 \quad-2.715500 \quad-0.8835910 .007816$

$6 \quad-4.102895 \quad-0.886190 \quad 0.048254$

$\begin{array}{llll}1 & -4.682050 & 0.033713 & 0.039790\end{array}$ S 60 


$\begin{array}{llll}6 & -4.750038 & -2.119732 & 0.113114 \\ 1 & -5.833857 & -2.158692 & 0.145084 \\ 6 & -4.016178 & -3.308761 & 0.142805 \\ 1 & -4.542475 & -4.256541 & 0.194874 \\ 6 & -2.621689 & -3.297311 & 0.111602 \\ 1 & -2.065140 & -4.229311 & 0.140360 \\ 6 & -1.975955 & -2.069955 & 0.044802 \\ 6 & -0.551591 & -1.655794 & -0.005121 \\ 6 & 1.918514 & -2.272873 & -0.050249 \\ 6 & 2.792769 & -3.356591 & 0.114093 \\ 1 & 2.396845 & -4.352818 & 0.283065 \\ 6 & 4.161764 & -3.130127 & 0.054677 \\ 1 & 4.862515 & -3.950197 & 0.177869 \\ 6 & 4.616576 & -1.839057 & -0.169828 \\ 1 & 5.677721 & -1.618245 & -0.228608 \\ 6 & 3.675178 & -0.812057 & -0.327507 \\ 6 & 2.024670 & 3.418936 & 1.289804 \\ 1 & 2.246801 & 2.354336 & 1.370129 \\ 1 & 1.953755 & 3.834064 & 2.300550 \\ 1 & 2.842714 & 3.930706 & 0.775933 \\ 6 & 4.116134 & 0.609377 & -0.563087 \\ 1 & 4.498157 & 1.051280 & 0.363514 \\ 1 & 3.261872 & 1.197718 & -0.899701 \\ 1 & 4.915935 & 0.660815 & -1.307052 \\ 6 & 0.478552 & -2.528768 & 0.008330 \\ 1 & 0.209249 & -3.580775 & 0.078127 \\ 7 & -2.068568 & 1.498706 & -0.133261 \\ 1 & -3.033474 & 1.663642 & -0.375391 \\ 0 & -1.446887 & -0.938338 & -0.015034\end{array}$
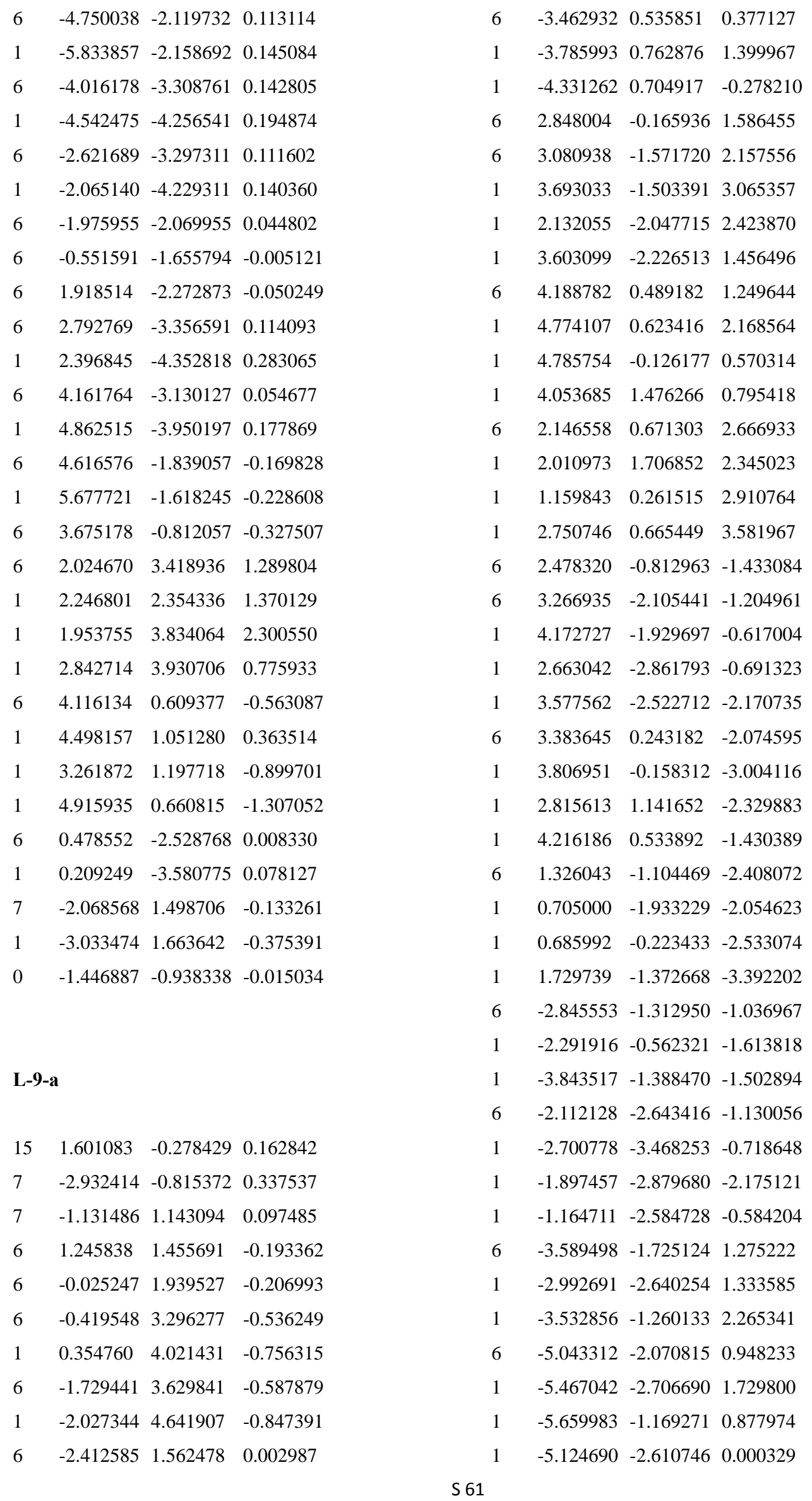


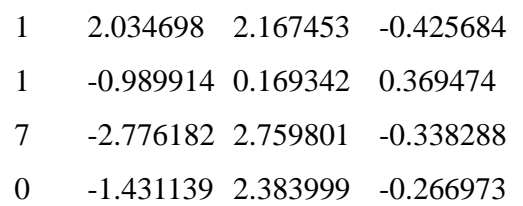

\section{L-9-b}

$15 \quad 1.703899 \quad-0.166362 \quad-0.305401$

$\begin{array}{lllll}7 & -3.097990 & -0.625173 & 0.088230\end{array}$

$\begin{array}{llll}7 & -0.981523 & 1.150597 & 0.477334\end{array}$

$\begin{array}{lllll}6 & 1.410880 & 1.324326 & 0.811822\end{array}$

$\begin{array}{lllll}6 & 0.078836 & 1.963261 & 0.532504\end{array}$

$\begin{array}{lllll}6 & -0.060984 & 3.332815 & 0.317531\end{array}$

$\begin{array}{llll}1 & 0.787998 & 4.005625 & 0.358305\end{array}$

$\begin{array}{lllll}6 & -1.342488 & 3.792763 & 0.037960\end{array}$

$1 \quad-1.527535 \quad 4.850067 \quad-0.142858$

$\begin{array}{llll}6 & -2.161630 & 1.688236 & 0.191258\end{array}$

$\begin{array}{llll}6 & -3.383224 & 0.793845 & 0.120451\end{array}$

$\begin{array}{llll}1 & -3.986284 & 1.012942 & 1.011137\end{array}$

$\begin{array}{llll}1 & -3.976407 & 1.145610 & -0.742949\end{array}$

$\begin{array}{lllll}6 & 1.601687 & -1.619245 & 0.913796\end{array}$

$\begin{array}{lllll}6 & 1.565898 & -2.906536 & 0.074155\end{array}$

$\begin{array}{lllll}1 & 1.400720 & -3.767221 & 0.733652\end{array}$

$1 \quad 0.749865 \quad-2.874724 \quad-0.654126$

$1 \quad 2.498220 \quad-3.080315 \quad-0.468696$

$\begin{array}{lllll}6 & 2.721319 & -1.713917 & 1.950850\end{array}$

$\begin{array}{lllll}1 & 2.482496 & -2.502386 & 2.675658\end{array}$

$\begin{array}{lllll}1 & 3.681047 & -1.969901 & 1.493567\end{array}$

$\begin{array}{lllll}1 & 2.842466 & -0.778916 & 2.508672\end{array}$

$\begin{array}{lllll}6 & 0.254975 & -1.508150 & 1.648554\end{array}$

$\begin{array}{lllll}1 & 0.253906 & -0.696668 & 2.383247\end{array}$

$\begin{array}{lllll}1 & -0.588521 & -1.321021 & 0.977544\end{array}$

$\begin{array}{lllll}1 & 0.078804 & -2.444056 & 2.193626\end{array}$

$\begin{array}{lllll}6 & 3.525521 & 0.147704 & -0.762683\end{array}$

$\begin{array}{lllll}6 & 4.097209 & -1.113375 & -1.422893\end{array}$

$1 \quad 4.304877 \quad-1.896940 \quad-0.688099$

$\begin{array}{lllll}1 & 3.414189 & -1.518925 & -2.177048\end{array}$

$\begin{array}{lllll}1 & 5.044239 & -0.871500 & -1.920648\end{array}$

$\begin{array}{lllll}6 & 4.485457 & 0.608920 & 0.340979\end{array}$

$1 \quad 5.465187 \quad 0.820797 \quad-0.105991$

$\begin{array}{llll}1 & 4.153008 & 1.529162 & 0.829492\end{array}$

$\begin{array}{llll}1 & 4.634759 & -0.147639 & 1.111718\end{array}$
$6 \quad 3.449346 \quad 1.252104 \quad-1.831616$

$1 \quad 2.849911 \quad 0.933673 \quad-2.689078$

$1 \quad 3.008573 \quad 2.175653 \quad-1.439173$

$1 \quad 4.458604 \quad 1.493784 \quad-2.186084$

$6 \quad-2.501596 \quad-1.014408-1.189697$

$1 \quad-1.558654-0.465812-1.284050$

$1 \quad-3.139284-0.711713-2.042164$

$6 \quad-2.205537 \quad-2.506132 \quad-1.281924$

$1 \quad-3.114274-3.106580-1.385665$

$1 \quad-1.576289-2.704069-2.153588$

$1 \quad-1.671769-2.845946 \quad-0.389009$

$\begin{array}{lllll}6 & -4.259241 & -1.422445 & 0.476912\end{array}$

$1 \quad-3.930145 \quad-2.456781 \quad 0.617071$

$1 \quad-4.577327 \quad-1.068811 \quad 1.464266$

$6 \quad-5.451808-1.390785-0.484394$

$1 \quad-6.265405-2.011129-0.098706$

$1 \quad-5.838588 \quad-0.375597 \quad-0.613037$

$1 \quad-5.179737 \quad-1.774353-1.472571$

$\begin{array}{llll}1 & 1.456068 & 1.020294 & 1.864210\end{array}$

$\begin{array}{llll}1 & 2.201038 & 2.062124 & 0.658831\end{array}$

$\begin{array}{llll}7 & -2.397911 & 2.986406 & -0.038110\end{array}$

$\begin{array}{llll}0 & -1.144424 & 2.452142 & 0.254259\end{array}$

\section{L-10-a}

$\begin{array}{llll}15 & 1.609193 & -0.290148 & 0.212529\end{array}$

$\begin{array}{lllll}7 & -2.982925 & -0.754344 & 0.383061\end{array}$

$\begin{array}{llll}7 & -1.112187 & 1.139392 & 0.141078\end{array}$

$\begin{array}{llll}6 & 1.268751 & 1.455381 & -0.113011\end{array}$

$\begin{array}{lllll}6 & 0.006663 & 1.942011 & -0.141983\end{array}$

$\begin{array}{llll}6 & -1.530134 & 3.592985 & -0.573903\end{array}$

$1 \quad-1.744370 \quad 4.630484 \quad-0.826152$

$\begin{array}{lllll}6 & -2.363255 & 1.583766 & -0.040473\end{array}$

$\begin{array}{llll}6 & -3.476371 & 0.604819 & 0.263542\end{array}$

$\begin{array}{llll}1 & -3.919247 & 0.919571 & 1.215516\end{array}$

$1 \quad-4.250701 \quad 0.744950-0.506704$

$\begin{array}{llll}6 & 2.982603 & -0.194410 & 1.512432\end{array}$

$\begin{array}{lllll}6 & 3.234670 & -1.603730 & 2.067119\end{array}$

$\begin{array}{lllll}1 & 3.949640 & -1.548441 & 2.897126\end{array}$

$\begin{array}{lllll}1 & 2.308755 & -2.047449 & 2.446037\end{array}$

$\begin{array}{llll}1 & 3.651999 & -2.279446 & 1.317003\end{array}$

$\begin{array}{llll}6 & 4.301895 & 0.421620 & 1.043794\end{array}$

S 62 


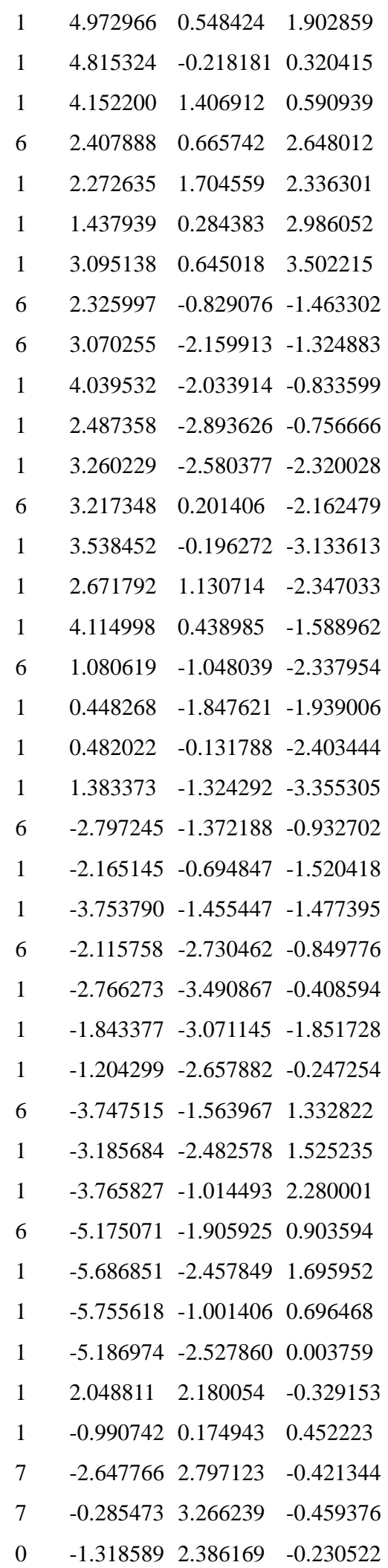

\section{L-10-b}

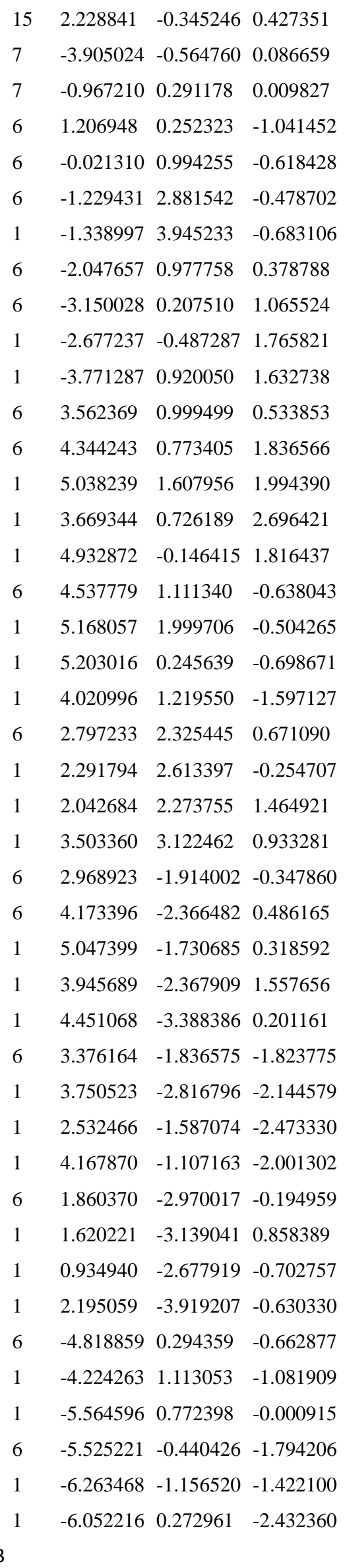




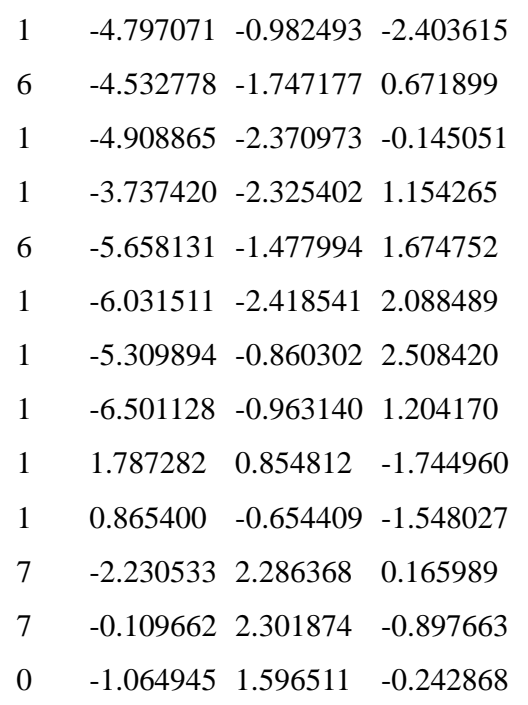

\section{L-11-a}

$15 \quad 2.050540 \quad-0.111549 \quad 0.789070$

$\begin{array}{llll}7 & -3.830772 & -0.342611 & 0.675330\end{array}$

$\begin{array}{lllll}7 & -0.958409 & 0.270764 & -0.221985\end{array}$

$\begin{array}{lllll}6 & 1.238983 & -0.521938 & -0.875773\end{array}$

$\begin{array}{lllll}6 & -0.019727 & 0.265611 & -1.168609\end{array}$

$\begin{array}{lllll}6 & -0.173704 & 0.928736 & -2.389978\end{array}$

$\begin{array}{llll}1 & 0.610625 & 0.876283 & -3.139317\end{array}$

$\begin{array}{lllll}6 & -1.333563 & 1.661211 & -2.608562\end{array}$

$\begin{array}{llll}1 & -1.483295 & 2.197793 & -3.540394\end{array}$

$\begin{array}{llll}6 & -2.299120 & 1.691114 & -1.608735\end{array}$

$1 \quad-3.219090 \quad 2.255242 \quad-1.729064$

$\begin{array}{lllll}6 & -2.077953 & 0.965996 & -0.435331\end{array}$

$\begin{array}{llll}6 & -3.105943 & 0.920883 & 0.673573\end{array}$

$\begin{array}{llll}1 & -2.566320 & 1.004990 & 1.621775\end{array}$

$\begin{array}{llll}1 & -3.778339 & 1.795346 & 0.585354\end{array}$

$\begin{array}{lllll}6 & 2.614430 & 1.693554 & 0.527445\end{array}$

$\begin{array}{llll}6 & 3.317482 & 2.162239 & 1.814199\end{array}$

$\begin{array}{llll}1 & 3.465192 & 3.247651 & 1.761865\end{array}$

$\begin{array}{llll}1 & 2.704206 & 1.949323 & 2.695790\end{array}$

$\begin{array}{llll}1 & 4.296551 & 1.707945 & 1.961379\end{array}$

$\begin{array}{lllll}6 & 3.513933 & 1.958982 & -0.681697\end{array}$

$\begin{array}{llll}1 & 3.715790 & 3.034885 & -0.764516\end{array}$

$1 \quad 4.477961 \quad 1.449712 \quad-0.597853$

$1 \quad 3.038894 \quad 1.643306 \quad-1.617292$

$\begin{array}{llll}6 & 1.346107 & 2.561971 & 0.405574\end{array}$

$\begin{array}{llll}1 & 0.873741 & 2.481369 & -0.574393\end{array}$ $\begin{array}{llll}1 & 0.602461 & 2.299718 & 1.165478\end{array}$

$\begin{array}{llll}1 & 1.625635 & 3.612639 & 0.549057\end{array}$

$\begin{array}{llll}6 & 3.576658 & -1.260192 & 0.628019\end{array}$

$\begin{array}{lllll}6 & 4.752648 & -0.729248 & 1.455348\end{array}$

$\begin{array}{llll}1 & 5.237042 & 0.132542 & 0.988717\end{array}$

$\begin{array}{lllll}1 & 4.446123 & -0.453200 & 2.469500\end{array}$

$1 \quad 5.507888-1.519975 \quad 1.542559$

$\begin{array}{lllll}6 & 4.061498 & -1.540458 & -0.798525\end{array}$

$\begin{array}{lllll}1 & 4.961343 & -2.166887 & -0.756171\end{array}$

$1 \quad 3.307709 \quad-2.088669-1.369461$

$1 \quad 4.320888 \quad-0.627497-1.342669$

$\begin{array}{lllll}6 & 3.139744 & -2.590225 & 1.269819\end{array}$

$\begin{array}{llll}1 & 2.876653 & -2.446508 & 2.322156\end{array}$

$\begin{array}{llll}1 & 2.278063 & -3.018101 & 0.755463\end{array}$

$\begin{array}{llll}1 & 3.971515 & -3.304723 & 1.219929\end{array}$

$6 \quad-4.796209-0.399541-0.414545$

$1 \quad-4.243743-0.221466-1.344300$

$1 \quad-5.546430 \quad 0.412599 \quad-0.342216$

$6 \quad-5.502299 \quad-1.745079 \quad-0.516840$

$1 \quad-6.207844 \quad-1.9044860 .303383$

$1 \quad-6.065925-1.802221-1.451276$

$1 \quad-4.767782 \quad-2.554768 \quad-0.502170$

$6 \quad-4.378705 \quad-0.672826 \quad 1.987729$

$1 \quad-4.720582-1.712316 \quad 1.962084$

$1 \quad-3.544757 \quad-0.645538 \quad 2.696895$

$\begin{array}{lllll}6 & -5.511888 & 0.228589 & 2.487639\end{array}$

$1 \quad-5.820676 \quad-0.071994 \quad 3.492459$

$\begin{array}{llll}1 & -5.198418 & 1.276145 & 2.534250\end{array}$

$1 \quad-6.3910690 .169458 \quad 1.838542$

$1 \quad 1.947340 \quad-0.356179 \quad-1.705033$

$8 \quad 0.951398 \quad-1.906327 \quad-0.783425$

$6 \quad 0.512220 \quad-2.467945 \quad-1.998320$

$1 \quad 0.398349 \quad-3.540037-1.830645$

$1 \quad-0.451232 \quad-2.049941-2.316279$

$1 \quad 1.248949 \quad-2.304259 \quad-2.800238$

$\begin{array}{llll}0 & -1.140578 & 0.941391 & -1.412506\end{array}$

\section{L-11-b}

$15 \quad-1.545064 \quad-0.582184-0.529789$

$\begin{array}{lllll}7 & 3.259978 & -0.599864 & -0.377819\end{array}$

$\begin{array}{llll}7 & 1.042349 & 1.062071 & -0.360927\end{array}$ 

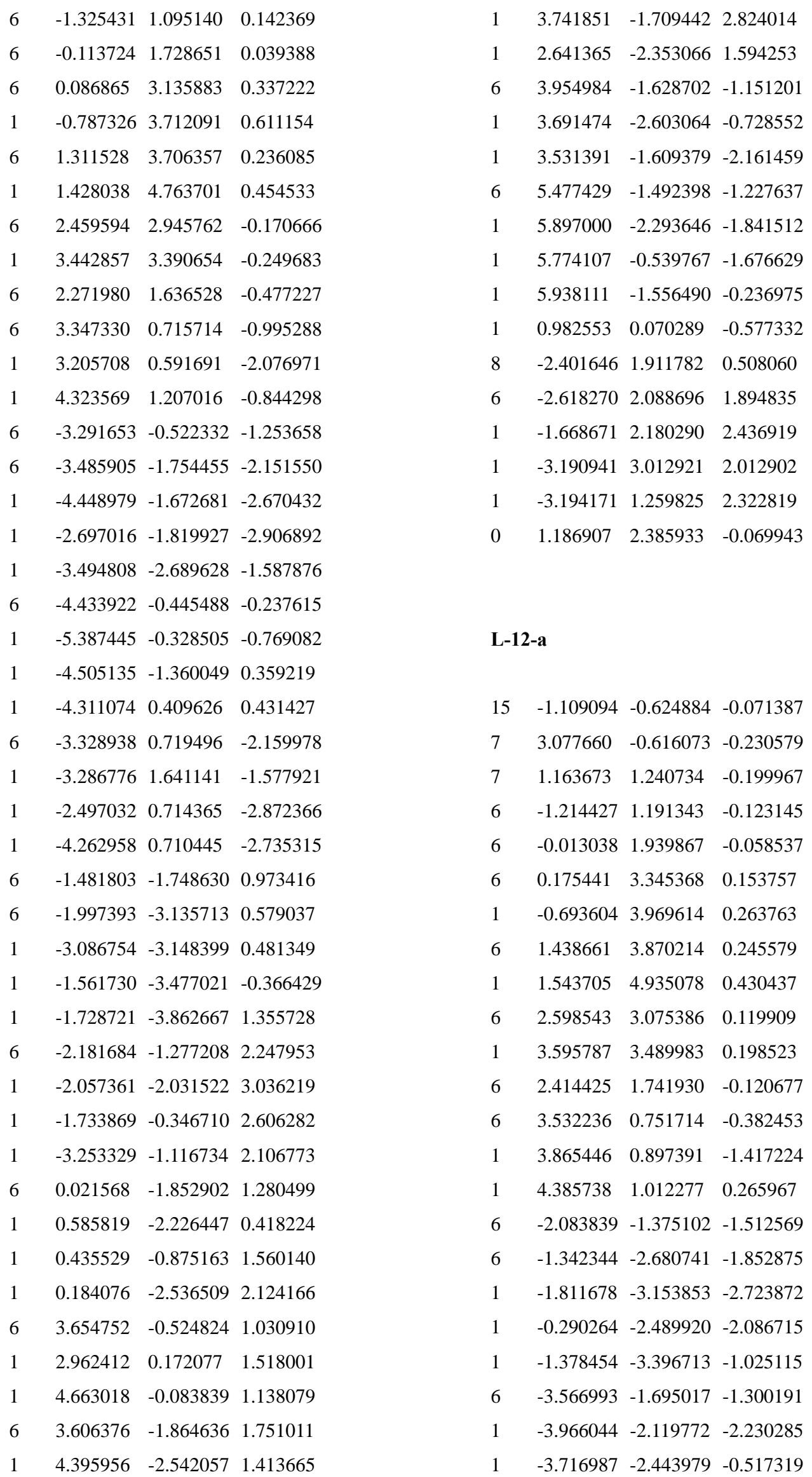

\section{L-12-a}

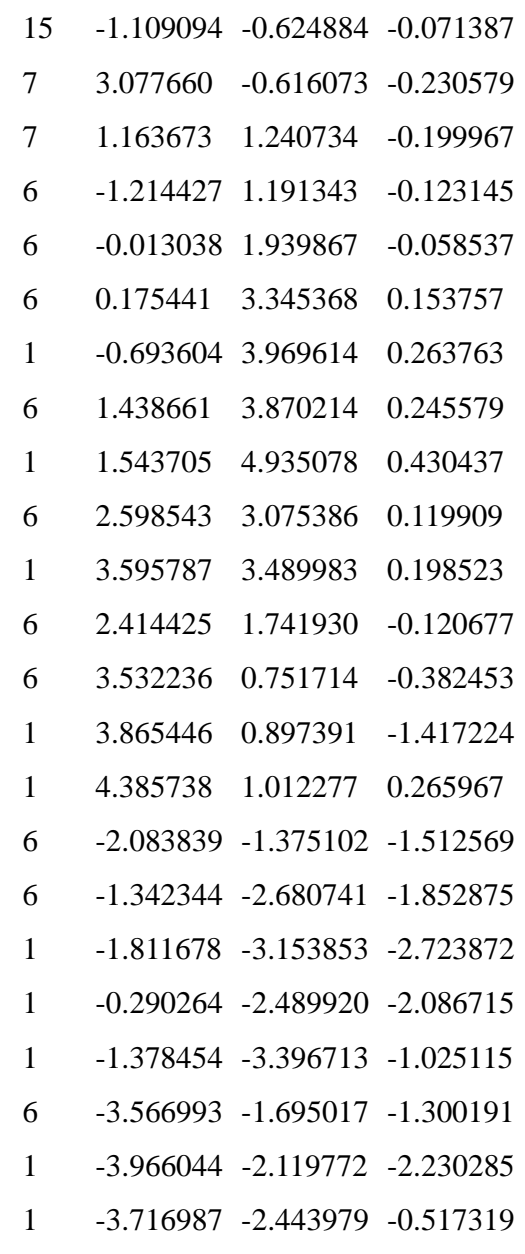




$$
\begin{array}{llll}
1 & -4.139299 & -0.802462 & -1.052579 \\
6 & -1.940265 & -0.408276 & -2.694485 \\
1 & -2.535460 & 0.492918 & -2.532002 \\
1 & -0.895707 & -0.118584 & -2.857950 \\
1 & -2.297020 & -0.900998 & -3.606935 \\
6 & -1.830523 & -1.111009 & 1.608043 \\
6 & -1.784783 & -2.641888 & 1.712027 \\
1 & -2.499765 & -3.123659 & 1.039877 \\
1 & -0.785640 & -3.031438 & 1.487679 \\
1 & -2.040513 & -2.942959 & 2.735029 \\
6 & -3.235902 & -0.607221 & 1.947347 \\
1 & -3.514187 & -0.985268 & 2.940042 \\
1 & -3.274724 & 0.483349 & 1.978286 \\
1 & -3.985712 & -0.942707 & 1.230153 \\
6 & -0.846474 & -0.532388 & 2.637395 \\
1 & 0.165157 & -0.924706 & 2.486410 \\
1 & -0.808653 & 0.560229 & 2.580647 \\
1 & -1.170675 & -0.807414 & 3.648066 \\
6 & 3.017938 & -1.011477 & 1.178461 \\
1 & 2.402092 & -0.263511 & 1.694280 \\
1 & -2.450 \\
1 & 4.015731 & -0.971335 & 1.650046 \\
6 & 2.392673 & -2.386079 & 1.368719 \\
1 & 3.054864 & -3.188751 & 1.032165 \\
1 & 2.176378 & -2.557358 & 2.426235 \\
1 & 1.455473 & -2.441150 & 0.805259 \\
6 & 3.768028 & -1.562685 & -1.106622 \\
1 & 3.209534 & -2.502884 & -1.091898 \\
6 & 3.684052 & -1.175011 & -2.127464 \\
1 & 5.236631 & -1.819946 & -0.766717 \\
\hline 1 & 5.681998 & -2.497472 & -1.499529 \\
\hline 16488 & -0.891515 & -0.773642 \\
\hline 1 & -289994 & 2.533767 & 0.017375
\end{array}
$$

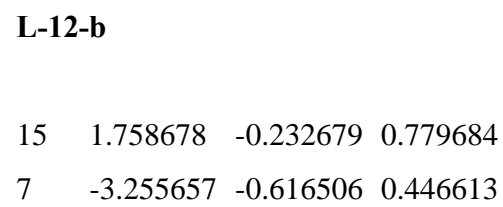$$
\text { L-12-b }
$$$$
\begin{array}{lllll}
15 & 1.758678 & -0.232679 & 0.779684
\end{array}
$$$$
\begin{array}{lllll}
7 & -3.255657 & -0.616506 & 0.446613
\end{array}
$$

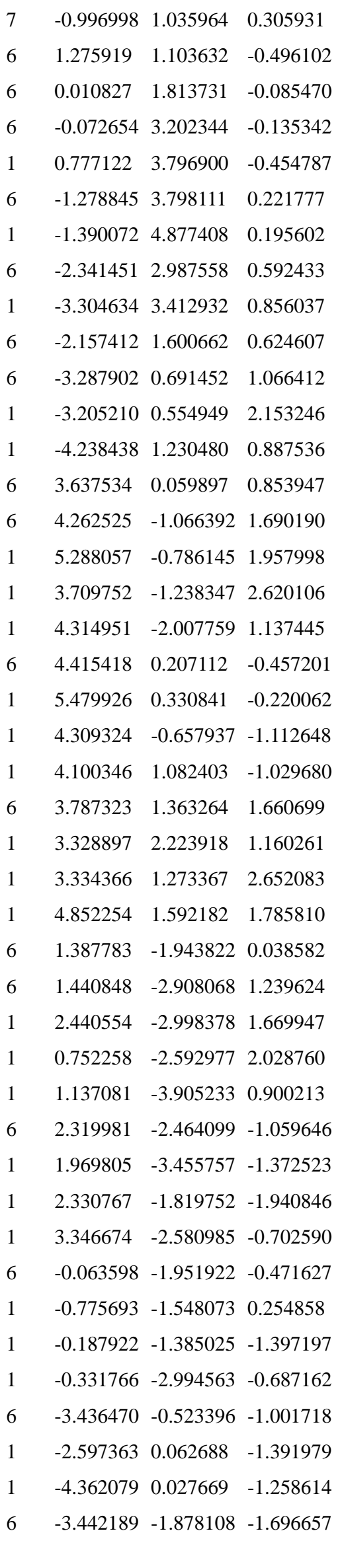




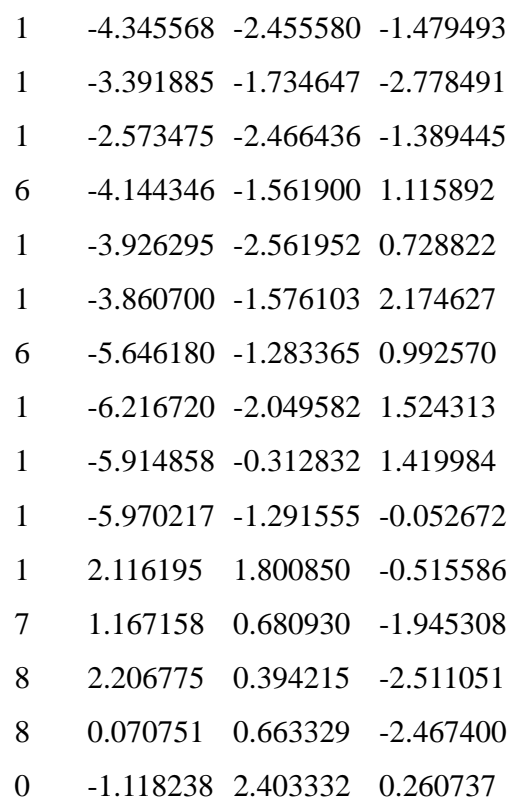

\section{L-13-a}

$7 \quad-1.996780 \quad-0.427427 \quad-0.317317$

$\begin{array}{lllll}7 & 0.101705 & 1.426464 & 0.134007\end{array}$

$\begin{array}{llll}7 & 1.986749 & -0.515013 & 0.181709\end{array}$

$6 \quad-2.459169-1.640367-0.636680$

$6 \quad-3.738228-2.061681-0.263175$

$1 \quad-4.082001 \quad-3.054132-0.536070$

$\begin{array}{llll}6 & -4.550715 & -1.191758 & 0.450935\end{array}$

$\begin{array}{llll}1 & -5.549544 & -1.492390 & 0.751448\end{array}$

$\begin{array}{llll}6 & -4.071525 & 0.074353 & 0.766608\end{array}$

$\begin{array}{llll}1 & -4.680499 & 0.787511 & 1.313369\end{array}$

$\begin{array}{lllll}6 & -2.783501 & 0.412654 & 0.357021\end{array}$

$\begin{array}{lllll}6 & -0.700427 & 3.433625 & -0.336071\end{array}$

$\begin{array}{lllll}6 & 0.645965 & 3.544174 & -0.384212\end{array}$

$\begin{array}{lllll}6 & 1.189373 & 2.218656 & -0.157811\end{array}$

$\begin{array}{lllll}6 & 2.920808 & 0.440872 & -0.051245\end{array}$

$\begin{array}{lllll}6 & 4.293127 & 0.105886 & -0.104679\end{array}$

$\begin{array}{lllll}1 & 5.023872 & 0.883642 & -0.300717\end{array}$

$\begin{array}{lllll}6 & 4.679743 & -1.201778 & 0.105162\end{array}$

$\begin{array}{lllll}1 & 5.731380 & -1.472736 & 0.073338\end{array}$

$\begin{array}{lllll}6 & 3.710866 & -2.170993 & 0.370426\end{array}$

$\begin{array}{llll}1 & 3.978898 & -3.205523 & 0.556753\end{array}$

$\begin{array}{lllll}6 & 2.375600 & -1.775416 & 0.394892\end{array}$

$6 \quad-1.545296 \quad-2.527921 \quad-1.437621$

$1 \quad-0.567919-2.053035-1.534686$
$1 \quad-1.956828-2.697678-2.437434$

$1 \quad-1.423402 \quad-3.504102 \quad-0.959199$

$\begin{array}{lllll}6 & 1.284294 & -2.779996 & 0.669234\end{array}$

$1 \quad 1.159282-3.463289-0.177668$

$\begin{array}{lllll}1 & 0.339099 & -2.259942 & 0.839514\end{array}$

$\begin{array}{lllll}1 & 1.523015 & -3.386960 & 1.547170\end{array}$

$\begin{array}{lllll}7 & -1.078282 & 2.123150 & -0.144401\end{array}$

$\begin{array}{llll}6 & -2.211147 & 1.776355 & 0.695073\end{array}$

$\begin{array}{lllll}1 & 3.243785 & 2.547110 & -0.461195\end{array}$

$\begin{array}{llll}1 & -2.980431 & 2.538743 & 0.540354\end{array}$

$\begin{array}{llll}1 & -1.917630 & 1.800657 & 1.755299\end{array}$

$\begin{array}{lllll}1 & -1.464434 & 4.191908 & -0.442367\end{array}$

$\begin{array}{lllll}1 & 1.222755 & 4.426943 & -0.611109\end{array}$

$\begin{array}{lllll}6 & 2.492658 & 1.798591 & -0.240366\end{array}$

$\begin{array}{lllll}1 & 0.112308 & 0.431504 & -0.079728\end{array}$

$\begin{array}{lllll}0 & 0.012433 & 2.559638 & -0.164690\end{array}$

\section{L-13-b}

$\begin{array}{llll}7 & 4.486153 & -0.922599 & -0.028069\end{array}$

$\begin{array}{lllll}7 & 0.068563 & -0.220413 & 0.429544\end{array}$

$\begin{array}{lllll}7 & -4.390458 & 0.927796 & 0.166099\end{array}$

$\begin{array}{lllll}6 & 5.564397 & -0.161802 & 0.193605\end{array}$

$\begin{array}{llll}6 & 5.463273 & 1.205548 & 0.457063\end{array}$

$\begin{array}{llll}1 & 6.359555 & 1.792407 & 0.627928\end{array}$

$\begin{array}{lllll}6 & 4.203947 & 1.791846 & 0.495126\end{array}$

$\begin{array}{llll}1 & 4.097482 & 2.852748 & 0.699623\end{array}$

$\begin{array}{llll}6 & 3.081792 & 1.004371 & 0.270895\end{array}$

$\begin{array}{llll}1 & 2.078256 & 1.414502 & 0.303489\end{array}$

$\begin{array}{llll}6 & 3.282826 & -0.351006 & 0.012515\end{array}$

$\begin{array}{lllll}6 & 0.518058 & -0.135034 & -1.763208\end{array}$

$\begin{array}{lllll}6 & -0.626682 & 0.601721 & -1.561485\end{array}$

$\begin{array}{lllll}6 & -0.862434 & 0.516468 & -0.169576\end{array}$

$\begin{array}{llll}6 & -3.276638 & 0.261853 & 0.469488\end{array}$

$\begin{array}{lllll}6 & -3.249540 & -1.122719 & 0.666409\end{array}$

$\begin{array}{lllll}1 & -2.309643 & -1.611596 & 0.902523\end{array}$

$\begin{array}{lllll}6 & -4.436607 & -1.827703 & 0.546981\end{array}$

$\begin{array}{lllll}1 & -4.457781 & -2.903175 & 0.695387\end{array}$

$\begin{array}{lllll}6 & -5.603501 & -1.136008 & 0.232042\end{array}$

$1 \quad-6.551241 \quad-1.653648 \quad 0.127396$

$\begin{array}{llll}6 & -5.537701 & 0.243631 & 0.047619\end{array}$

$\begin{array}{llll}6 & 6.895189 & -0.861527 & 0.137752\end{array}$ 


$$
\begin{array}{llll}
1 & 6.929403 & -1.661483 & 0.881972 \\
1 & 7.720562 & -0.171683 & 0.323049 \\
1 & 7.034438 & -1.324430 & -0.842565 \\
6 & -6.756669 & 1.054846 & -0.301112 \\
1 & -6.617223 & 1.544646 & -1.268558 \\
1 & -6.906917 & 1.842052 & 0.442001 \\
1 & -7.654142 & 0.434801 & -0.346574 \\
7 & 0.890465 & -0.620049 & -0.554691 \\
6 & 2.124294 & -1.299529 & -0.227964 \\
1 & -1.716121 & 1.111488 & 1.669210 \\
1 & 2.387838 & -1.982613 & -1.039060 \\
1 & 1.936304 & -1.894386 & 0.669450 \\
1 & 1.092479 & -0.352886 & -2.651939 \\
1 & -1.221462 & 1.115661 & -2.300532 \\
6 & -2.008833 & 1.080730 & 0.614180 \\
1 & -2.235883 & 2.100328 & 0.295037 \\
0 & -0.011334 & 0.058823 & -0.734965
\end{array}
$$

\section{L-14-a}

$$
\begin{array}{llll}
7 & -2.008905 & -0.481509 & -0.181260 \\
7 & 0.145308 & 1.354532 & 0.113297 \\
7 & 2.492779 & 1.763731 & -0.322625 \\
7 & 1.995777 & -0.522369 & 0.150174 \\
6 & -2.536875 & -1.649918 & -0.556732 \\
6 & -3.885415 & -1.951606 & -0.340260 \\
1 & -4.281450 & -2.910570 & -0.658270 \\
6 & -4.695803 & -1.008684 & 0.274633 \\
1 & -5.746503 & -1.215764 & 0.451875 \\
6 & -4.146392 & 0.214830 & 0.644577 \\
1 & -4.751535 & 0.986808 & 1.109098 \\
6 & -2.795242 & 0.432581 & 0.392090 \\
6 & -0.627259 & 3.377001 & -0.304659 \\
6 & 0.718049 & 3.449969 & -0.436661 \\
6 & 1.239775 & 2.116574 & -0.214019 \\
6 & 2.881828 & 0.464107 & -0.107136 \\
6 & 4.268617 & 0.195908 & -0.162097 \\
1 & 4.938558 & 1.017905 & -0.383096 \\
6 & 4.717015 & -1.084537 & 0.078296 \\
1 & 5.779852 & -1.307141 & 0.049792 \\
6 & 3.793364 & -2.092601 & 0.369860 \\
1 & 4.110590 & -3.108783 & 0.578188
\end{array}
$$

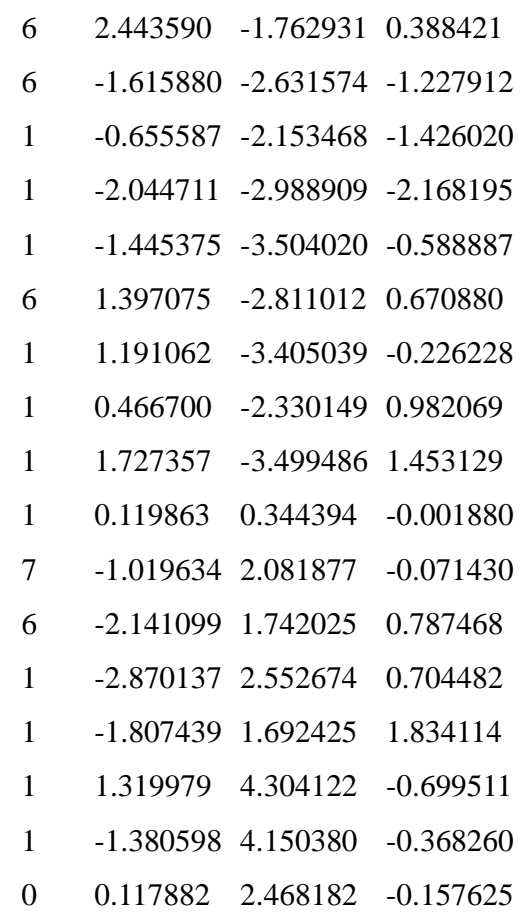

\section{L-14-b}

\begin{tabular}{llll}
7 & -4.059076 & -0.966663 & 0.316233 \\
7 & 0.269582 & 0.177059 & 0.324113 \\
7 & 2.181108 & 1.529347 & -0.121494 \\
7 & 3.182952 & -0.554624 & 0.156028 \\
6 & -5.083534 & -0.661451 & -0.488568 \\
6 & -4.994547 & 0.347629 & -1.449374 \\
1 & -5.847727 & 0.573029 & -2.080346 \\
6 & -3.801930 & 1.049261 & -1.578711 \\
1 & -3.704357 & 1.835204 & -2.321254 \\
6 & -2.733799 & 0.730880 & -0.749516 \\
1 & -1.778193 & 1.238232 & -0.824555 \\
6 & -2.919453 & -0.287854 & 0.184222 \\
6 & -0.656375 & 1.314196 & 2.022768 \\
6 & 0.486109 & 1.994898 & 1.668363 \\
6 & 1.024513 & 1.230662 & 0.606190 \\
6 & 3.243062 & 0.669898 & -0.352814 \\
6 & 4.336769 & 1.134154 & -1.110399 \\
1 & 4.341376 & 2.141884 & -1.513192 \\
6 & 5.387332 & 0.264612 & -1.324691 \\
1 & 6.248469 & 0.585905 & -1.903058 \\
6 & 5.330954 & -1.027803 & -0.800912 \\
1 & 6.136506 & -1.735713 & -0.957412 \\
8 & & & \\
\hline & & & \\
\hline
\end{tabular}




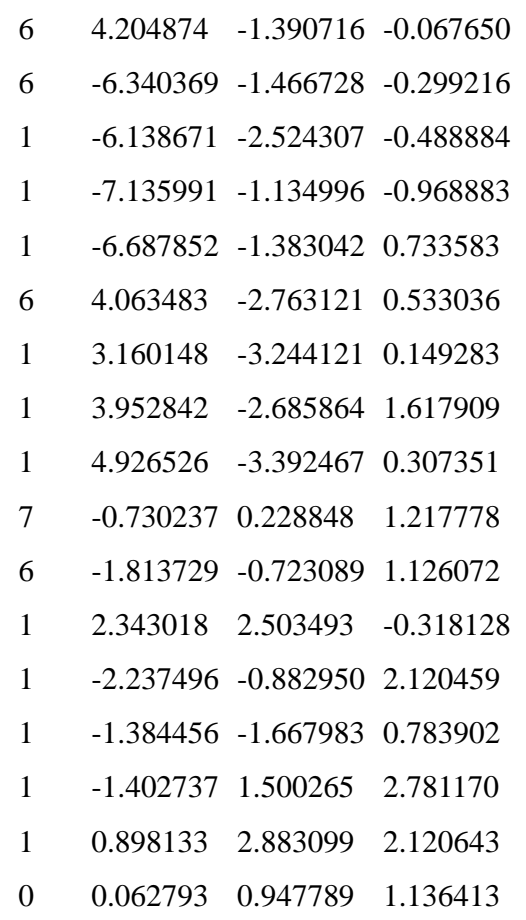

\section{L-15-a}

$\begin{array}{lllll}7 & 0.982099 & -2.102634 & -0.421955\end{array}$

$\begin{array}{llll}7 & -0.609872 & 0.149193 & 0.196470\end{array}$

$\begin{array}{llll}7 & 1.530031 & 1.839622 & 0.143104\end{array}$

$\begin{array}{lllll}6 & 2.164353 & -2.644556 & -0.736552\end{array}$

$\begin{array}{lllll}6 & 2.585067 & -3.861081 & -0.195950\end{array}$

$\begin{array}{lllll}1 & 3.553240 & -4.269127 & -0.467363\end{array}$

$\begin{array}{lllll}6 & 1.744891 & -4.529691 & 0.686224\end{array}$

$\begin{array}{llll}1 & 2.046456 & -5.476981 & 1.122577\end{array}$

$\begin{array}{lllll}6 & 0.512521 & -3.970808 & 0.998358\end{array}$

$\begin{array}{lllll}1 & -0.174595 & -4.463172 & 1.679712\end{array}$

$\begin{array}{lllll}6 & 0.172269 & -2.750371 & 0.414808\end{array}$

$\begin{array}{lllll}6 & -2.741321 & -0.445005 & -0.119763\end{array}$

$6 \quad-3.954946-1.128596-0.201343$

$1 \quad-3.996834-2.212059-0.229966$

$\begin{array}{lllll}6 & -5.117193 & -0.368988 & -0.268195\end{array}$

$1 \quad-6.074366-0.876095 \quad-0.338459$

$\begin{array}{llll}6 & -5.082705 & 1.031226 & -0.251757\end{array}$

$\begin{array}{llll}1 & -6.009653 & 1.591255 & -0.307311\end{array}$

$\begin{array}{lllll}6 & -3.869543 & 1.702423 & -0.158645\end{array}$

$\begin{array}{lllll}1 & -3.833309 & 2.787107 & -0.133344\end{array}$

$\begin{array}{llll}6 & -2.698434 & 0.954972 & -0.079756\end{array}$

$\begin{array}{llll}6 & -1.290914 & 1.333839 & 0.036720\end{array}$ $\begin{array}{llll}6 & 0.660689 & 2.873078 & 0.062960\end{array}$

$\begin{array}{lllll}6 & 1.117787 & 4.206856 & 0.067979\end{array}$

$\begin{array}{lllll}1 & 0.400725 & 5.017579 & -0.007803\end{array}$

$\begin{array}{llll}6 & 2.472138 & 4.455596 & 0.181536\end{array}$

$\begin{array}{llll}1 & 2.842405 & 5.476608 & 0.194240\end{array}$

$\begin{array}{llll}6 & 3.357105 & 3.383808 & 0.290129\end{array}$

$\begin{array}{lllll}1 & 4.425364 & 3.540644 & 0.395447\end{array}$

$\begin{array}{llll}6 & 2.836664 & 2.090782 & 0.260684\end{array}$

$\begin{array}{lllll}6 & 3.006406 & -1.890583 & -1.729835\end{array}$

$1 \quad 2.907982-2.337730-2.724390$

$1 \quad 4.064604 \quad-1.912823-1.456057$

$1 \quad 2.665926-0.855555 \quad-1.785547$

$\begin{array}{llll}6 & 3.747915 & 0.893281 & 0.352039\end{array}$

$1 \quad 4.259262 \quad 0.721751 \quad-0.601280$

$\begin{array}{llll}1 & 3.163615 & 0.003085 & 0.594844\end{array}$

$\begin{array}{llll}1 & 4.516557 & 1.040007 & 1.115580\end{array}$

$\begin{array}{lllll}1 & 0.345606 & 0.069124 & -0.141624\end{array}$

$7 \quad-1.446893 \quad-0.955806 \quad-0.089723$

$\begin{array}{lllll}6 & -1.160271 & -2.106561 & 0.748142\end{array}$

$1 \quad-1.955884-2.8403430 .588897$

$1 \quad-1.172914-1.820015 \quad 1.811361$

$\begin{array}{lllll}6 & -0.750553 & 2.584518 & -0.020371\end{array}$

$\begin{array}{llll}1 & -1.433281 & 3.419262 & -0.129262\end{array}$

$\begin{array}{llll}0 & -1.689005 & 0.157981 & -0.046266\end{array}$

\section{L-15-b}

$7 \quad 3.792859 \quad-1.420861-0.957642$

$7 \quad-0.548446-0.046150-0.260972$

$7 \quad-4.449272-0.028444-0.060142$

$\begin{array}{lllll}6 & 4.815825 & -1.803037 & -0.183363\end{array}$

$\begin{array}{lllll}6 & 4.767665 & -1.705855 & 1.208378\end{array}$

$\begin{array}{lllll}1 & 5.619697 & -2.016827 & 1.803449\end{array}$

$\begin{array}{lllll}6 & 3.619433 & -1.201994 & 1.808825\end{array}$

$\begin{array}{llll}1 & 3.557032 & -1.114271 & 2.889160\end{array}$

$\begin{array}{llll}6 & 2.554316 & -0.810670 & 1.008253\end{array}$

$\begin{array}{lllll}1 & 1.636403 & -0.413738 & 1.428861\end{array}$

$\begin{array}{lllll}6 & 2.695185 & -0.942330 & -0.373179\end{array}$

$\begin{array}{lllll}6 & 0.855576 & 1.696646 & -0.485474\end{array}$

$\begin{array}{lllll}6 & 1.945049 & 2.535867 & -0.768918\end{array}$

$\begin{array}{lllll}1 & 2.832008 & 2.166599 & -1.273364\end{array}$

$\begin{array}{llll}6 & 1.833877 & 3.850458 & -0.362407\end{array}$ 


\begin{tabular}{llll}
1 & 2.651855 & 4.536256 & -0.559003 \\
6 & 0.684691 & 4.331645 & 0.307215 \\
1 & 0.646117 & 5.374611 & 0.602897 \\
6 & -0.377621 & 3.496743 & 0.590564 \\
1 & -1.257695 & 3.867522 & 1.107587 \\
6 & -0.289989 & 2.152509 & 0.193574 \\
6 & -1.128936 & 0.993939 & 0.296334 \\
6 & -3.310349 & -0.276131 & 0.586019 \\
6 & -2.901634 & -1.568359 & 0.930149 \\
1 & -1.957075 & -1.718706 & 1.440498 \\
6 & -3.717955 & -2.632542 & 0.580889 \\
1 & -3.433928 & -3.650629 & 0.829381 \\
6 & -4.907109 & -2.377359 & -0.095947 \\
1 & -5.570600 & -3.185305 & -0.385971 \\
6 & -5.237214 & -1.058198 & -0.400042 \\
6 & 6.025801 & -2.339880 & -0.898469 \\
1 & 5.753653 & -3.222454 & -1.483219 \\
1 & 6.819521 & -2.611006 & -0.199913 \\
1 & 6.409810 & -1.593469 & -1.598444 \\
6 & -6.505557 & -0.705743 & -1.129585 \\
1 & -6.270119 & -0.147341 & -2.039163 \\
1 & -7.130573 & -0.060857 & -0.506098 \\
1 & -7.076993 & -1.596289 & -1.398392 \\
7 & 0.640357 & 0.377065 & -0.753483 \\
6 & 1.574872 & -0.561304 & -1.322309 \\
1 & 2.013046 & -0.147228 & -2.235342 \\
1 & 0.998060 & -1.447202 & -1.599871 \\
6 & -2.478712 & 0.930173 & 0.955640 \\
1 & -2.339808 & 0.961842 & 2.044801 \\
0 & -3.052179 & 1.819762 & 0.680942 \\
\hline & -0.099338 & 1.004359 & -0.137864
\end{tabular}

\section{L-16-a}

$7 \quad 0.699426 \quad-2.253271 \quad-0.812910$

$\begin{array}{llll}7 & -0.476322 & 0.293122 & 0.006369\end{array}$

$\begin{array}{llll}7 & -0.308314 & 2.703474 & 0.064319\end{array}$

$\begin{array}{llll}7 & 1.805719 & 1.605633 & -0.001710\end{array}$

$\begin{array}{lllll}6 & 1.967155 & -2.636324 & -0.986932\end{array}$

$\begin{array}{lllll}6 & 2.747849 & -3.116481 & 0.068010\end{array}$

$\begin{array}{lllll}1 & 3.773956 & -3.421514 & -0.108847\end{array}$

$\begin{array}{lllll}6 & 2.187879 & -3.171859 & 1.336351\end{array}$ $\begin{array}{llll}1 & 2.770724 & -3.525258 & 2.181406\end{array}$

$\begin{array}{lllll}6 & 0.872926 & -2.755919 & 1.519093\end{array}$

$\begin{array}{lllll}1 & 0.425934 & -2.775114 & 2.504702\end{array}$

$\begin{array}{llll}6 & 0.158831 & -2.308466 & 0.407656\end{array}$

$\begin{array}{lllll}6 & -2.668449 & 0.073099 & -0.308602\end{array}$

$\begin{array}{lllll}6 & -3.992229 & -0.349723 & -0.486569\end{array}$

$1 \quad-4.250288-1.387196 \quad-0.648427$

$\begin{array}{lllll}6 & -4.984663 & 0.622006 & -0.495498\end{array}$

$1 \quad-6.014594 \quad 0.309882-0.638952$

$\begin{array}{lllll}6 & -4.699444 & 1.987111 & -0.342393\end{array}$

$1 \quad-5.507435 \quad 2.709996-0.365756$

$\begin{array}{lllll}6 & -3.388507 & 2.404999 & -0.174148\end{array}$

$\begin{array}{llll}1 & -3.117541 & 3.449612 & -0.061983\end{array}$

$\begin{array}{lllll}6 & -2.391471 & 1.436119 & -0.145190\end{array}$

$\begin{array}{lllll}6 & -0.947928 & 1.572833 & -0.004074\end{array}$

$\begin{array}{llll}6 & 1.069961 & 2.727114 & 0.109525\end{array}$

$\begin{array}{llll}6 & 1.684717 & 3.989215 & 0.263184\end{array}$

$\begin{array}{lllll}1 & 1.049498 & 4.863297 & 0.339038\end{array}$

$\begin{array}{lllll}6 & 3.059748 & 4.062068 & 0.310674\end{array}$

$\begin{array}{lllll}1 & 3.553305 & 5.021720 & 0.433045\end{array}$

$\begin{array}{llll}6 & 3.815597 & 2.889589 & 0.200463\end{array}$

$\begin{array}{llll}1 & 4.898989 & 2.911204 & 0.237418\end{array}$

$\begin{array}{llll}6 & 3.144163 & 1.684973 & 0.041373\end{array}$

$\begin{array}{lllll}6 & 2.508427 & -2.525548 & -2.386180\end{array}$

$1 \quad 2.005485-3.241780 \quad-3.042270$

$1 \quad 3.582904 \quad-2.716658-2.418324$

$\begin{array}{lllll}1 & 2.308202 & -1.527099 & -2.782638\end{array}$

$\begin{array}{lllll}6 & 3.875798 & 0.376185 & -0.092808\end{array}$

$1 \quad 3.698160 \quad-0.057126-1.082994$

$\begin{array}{llll}1 & 3.499555 & -0.339813 & 0.643740\end{array}$

$\begin{array}{llll}1 & 4.952165 & 0.498661 & 0.044775\end{array}$

$1 \quad 0.483742 \quad 0.111856-0.276836$

$\begin{array}{llll}7 & -1.473031 & -0.652470 & -0.301410\end{array}$

$\begin{array}{lllll}6 & -1.319156 & -1.904188 & 0.492496\end{array}$

$\begin{array}{lllll}6 & -1.786009 & -1.680246 & 1.932107\end{array}$

$1 \quad-1.733074 \quad-2.604351 \quad 2.513240$

$1 \quad-1.178962 \quad-0.912191 \quad 2.419881$

$\begin{array}{llll}1 & -2.827830 & -1.350004 & 1.927659\end{array}$

$6 \quad-2.115756-3.032080-0.175821$

$1 \quad-3.187242-2.931307 \quad 0.002809$

$1 \quad-1.912960 \quad-3.039701 \quad-1.248557$

$\begin{array}{lllll}1 & -1.801669 & -3.989398 & 0.248979\end{array}$

$\begin{array}{llll}0 & -1.597023 & 0.562243 & -0.121651\end{array}$ S 70 


$$
\begin{aligned}
& \text { L-16-b } \\
& 7 \quad-2.996801-1.030964-0.405763 \\
& \begin{array}{llll}
7 & 0.310668 & 0.128526 & 0.283642
\end{array} \\
& \begin{array}{llll}
7 & 2.015568 & 1.139661 & -1.044337
\end{array} \\
& \begin{array}{lllll}
7 & 3.178993 & -0.321646 & 0.349962
\end{array} \\
& 6 \quad-3.431135-1.790211-1.413894 \\
& 6 \quad-2.949076-3.084483-1.626630 \\
& 1 \quad-3.320984-3.674011-2.458092 \\
& 6 \quad-1.989010-3.588342-0.760728 \\
& \begin{array}{llll}
1 & -1.588004 & -4.587148 & -0.903204
\end{array} \\
& \begin{array}{lllll}
6 & -1.535862 & -2.794316 & 0.286994
\end{array} \\
& \begin{array}{llll}
1 & -0.773759 & -3.157218 & 0.964673
\end{array} \\
& \begin{array}{llll}
6 & -2.071732 & -1.515729 & 0.426495
\end{array} \\
& \begin{array}{llll}
6 & -1.152297 & 1.831755 & 0.510924
\end{array} \\
& \begin{array}{llll}
6 & -2.222415 & 2.707715 & 0.793549
\end{array} \\
& \begin{array}{llll}
1 & -3.087465 & 2.401231 & 1.362577
\end{array} \\
& \begin{array}{llll}
6 & -2.139936 & 3.998582 & 0.311817
\end{array} \\
& \begin{array}{llll}
1 & -2.957419 & 4.682315 & 0.518663
\end{array} \\
& \begin{array}{lllll}
6 & -1.033772 & 4.462557 & -0.432568
\end{array} \\
& \begin{array}{llll}
1 & -1.013219 & 5.491445 & -0.774790
\end{array} \\
& \begin{array}{lllll}
6 & 0.015024 & 3.613719 & -0.714243
\end{array} \\
& \begin{array}{lllll}
1 & 0.881622 & 3.958263 & -1.271720
\end{array} \\
& \begin{array}{lllll}
6 & -0.052881 & 2.293218 & -0.249179
\end{array} \\
& \begin{array}{lllll}
6 & 0.812968 & 1.159170 & -0.343295
\end{array} \\
& \begin{array}{lllll}
6 & 3.129110 & 0.357214 & -0.790565
\end{array} \\
& \begin{array}{lllll}
6 & 4.169020 & 0.337561 & -1.741179
\end{array} \\
& \begin{array}{llll}
1 & 4.086137 & 0.903555 & -2.663523
\end{array} \\
& 6 \quad 5.284520 \quad-0.425676 \quad-1.461520 \\
& \begin{array}{lllll}
1 & 6.105951 & -0.465729 & -2.170553
\end{array} \\
& \begin{array}{lllll}
6 & 5.344977 & -1.147011 & -0.268332
\end{array} \\
& \begin{array}{lllll}
1 & 6.205231 & -1.758729 & -0.022385
\end{array} \\
& \begin{array}{lllll}
6 & 4.264941 & -1.062530 & 0.605176
\end{array} \\
& 6 \quad-4.482614 \quad-1.182253 \quad-2.302871 \\
& \begin{array}{llll}
1 & -5.442270 & -1.142709 & -1.778376
\end{array} \\
& 1 \quad-4.615966-1.757185-3.221392 \\
& \begin{array}{llll}
1 & -4.206436 & -0.156567 & -2.555473
\end{array} \\
& \begin{array}{llll}
6 & 4.244915 & -1.812362 & 1.909787
\end{array} \\
& \begin{array}{llll}
1 & 3.423403 & -2.534319 & 1.910459
\end{array} \\
& \begin{array}{lllll}
1 & 4.066782 & -1.118544 & 2.735101
\end{array} \\
& \begin{array}{llll}
1 & 5.182334 & -2.343714 & 2.084892
\end{array}
\end{aligned}
$$

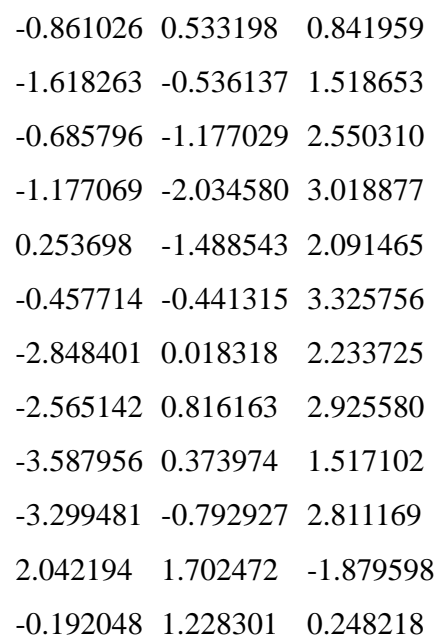



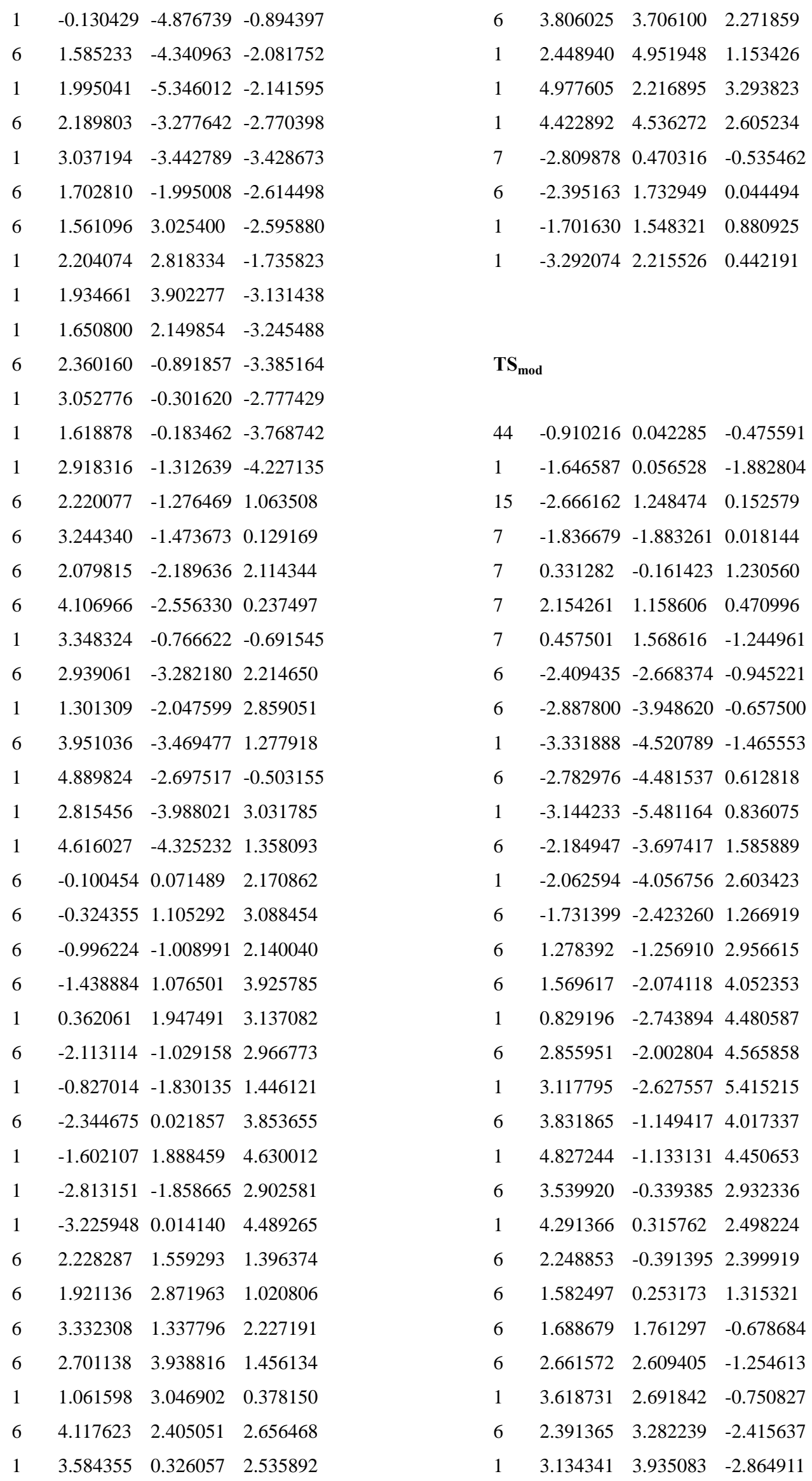

$\mathbf{T S}_{\bmod }$

$44 \quad-0.910216 \quad 0.042285 \quad-0.475591$

$\begin{array}{llll}1 & -1.646587 & 0.056528 & -1.882804\end{array}$

$\begin{array}{llll}15 & -2.666162 & 1.248474 & 0.152579\end{array}$

$\begin{array}{lllll}7 & -1.836679 & -1.883261 & 0.018144\end{array}$

$\begin{array}{llll}7 & 0.331282 & -0.161423 & 1.230560\end{array}$

$\begin{array}{llll}7 & 2.154261 & 1.158606 & 0.470996\end{array}$

$\begin{array}{llll}7 & 0.457501 & 1.568616 & -1.244961\end{array}$

$\begin{array}{lllll}6 & -2.409435 & -2.668374 & -0.945221\end{array}$

$\begin{array}{lllll}6 & -2.887800 & -3.948620 & -0.657500\end{array}$

$1 \quad-3.331888-4.520789-1.465553$

$\begin{array}{lllll}6 & -2.782976 & -4.481537 & 0.612818\end{array}$

$\begin{array}{lllll}1 & -3.144233 & -5.481164 & 0.836075\end{array}$

$\begin{array}{lllll}6 & -2.184947 & -3.697417 & 1.585889\end{array}$

$\begin{array}{lllll}1 & -2.062594 & -4.056756 & 2.603423\end{array}$

$\begin{array}{llll}6 & -1.731399 & -2.423260 & 1.266919\end{array}$

$\begin{array}{lllll}6 & 1.278392 & -1.256910 & 2.956615\end{array}$

$\begin{array}{lllll}6 & 1.569617 & -2.074118 & 4.052353\end{array}$

$\begin{array}{lllll}1 & 0.829196 & -2.743894 & 4.480587\end{array}$

$\begin{array}{lllll}6 & 2.855951 & -2.002804 & 4.565858\end{array}$

$\begin{array}{lllll}1 & 3.117795 & -2.627557 & 5.415215\end{array}$

$\begin{array}{lllll}6 & 3.831865 & -1.149417 & 4.017337\end{array}$

$\begin{array}{llll}1 & 4.827244 & -1.133131 & 4.450653\end{array}$

$\begin{array}{lllll}6 & 3.539920 & -0.339385 & 2.932336\end{array}$

$\begin{array}{llll}1 & 4.291366 & 0.315762 & 2.498224\end{array}$

$\begin{array}{lllll}6 & 2.248853 & -0.391395 & 2.399919\end{array}$

$\begin{array}{llll}6 & 1.582497 & 0.253173 & 1.315321\end{array}$

$\begin{array}{lllll}6 & 1.688679 & 1.761297 & -0.678684\end{array}$

$\begin{array}{lllll}6 & 2.661572 & 2.609405 & -1.254613\end{array}$

$\begin{array}{lllll}1 & 3.618731 & 2.691842 & -0.750827\end{array}$

$\begin{array}{lllll}6 & 2.391365 & 3.282239 & -2.415637\end{array}$

$\begin{array}{llll}1 & 3.134341 & 3.935083 & -2.864911\end{array}$

S 72 


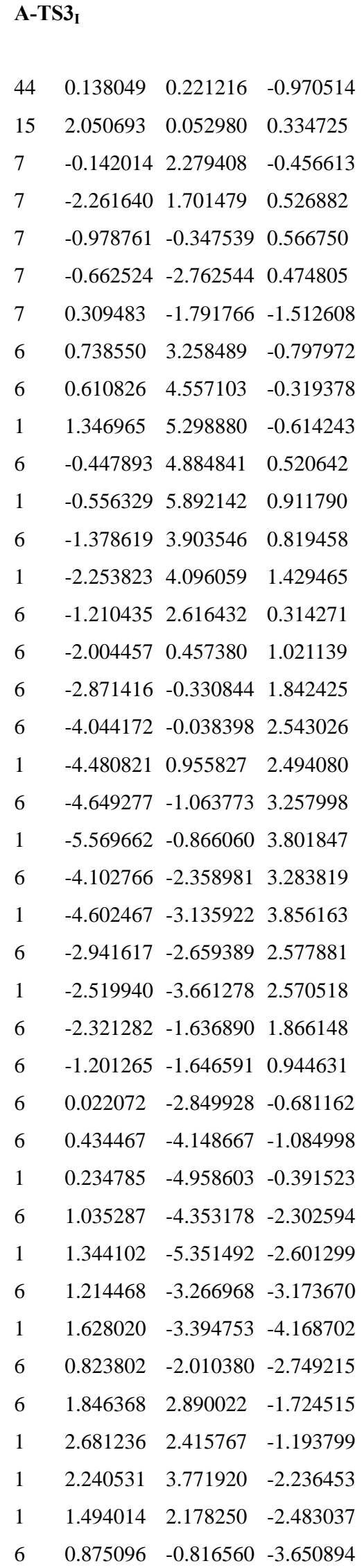

A-TS3 $_{\text {I }}$

$44 \quad 0.138049 \quad 0.221216 \quad-0.970514$

$\begin{array}{llll}15 & 2.050693 & 0.052980 & 0.334725\end{array}$

$\begin{array}{llll}7 & -0.142014 & 2.279408 & -0.456613\end{array}$

$\begin{array}{llll}7 & -2.261640 & 1.701479 & 0.526882\end{array}$

$\begin{array}{llll}7 & -0.978761 & -0.347539 & 0.566750\end{array}$

$\begin{array}{lllll}7 & -0.662524 & -2.762544 & 0.474805\end{array}$

$\begin{array}{lllll}7 & 0.309483 & -1.791766 & -1.512608\end{array}$

$\begin{array}{lllll}6 & 0.738550 & 3.258489 & -0.797972\end{array}$

$\begin{array}{lllll}6 & 0.610826 & 4.557103 & -0.319378\end{array}$

$\begin{array}{llll}1 & 1.346965 & 5.298880 & -0.614243\end{array}$

$\begin{array}{llll}6 & -0.447893 & 4.884841 & 0.520642\end{array}$

$\begin{array}{llll}1 & -0.556329 & 5.892142 & 0.911790\end{array}$

$\begin{array}{llll}6 & -1.378619 & 3.903546 & 0.819458\end{array}$

$\begin{array}{llll}1 & -2.253823 & 4.096059 & 1.429465\end{array}$

$\begin{array}{llll}6 & -1.210435 & 2.616432 & 0.314271\end{array}$

$\begin{array}{llll}6 & -2.004457 & 0.457380 & 1.021139\end{array}$

$6 \quad-2.871416 \quad-0.330844 \quad 1.842425$

$\begin{array}{llll}6 & -4.044172 & -0.038398 & 2.543026\end{array}$

$\begin{array}{llll}1 & -4.480821 & 0.955827 & 2.494080\end{array}$

$\begin{array}{lllll}6 & -4.649277 & -1.063773 & 3.257998\end{array}$

$\begin{array}{llll}1 & -5.569662 & -0.866060 & 3.801847\end{array}$

$\begin{array}{lllll}6 & -4.102766 & -2.358981 & 3.283819\end{array}$

$\begin{array}{llll}1 & -4.602467 & -3.135922 & 3.856163\end{array}$

$\begin{array}{lllll}6 & -2.941617 & -2.659389 & 2.577881\end{array}$

$\begin{array}{llll}1 & -2.519940 & -3.661278 & 2.570518\end{array}$

$\begin{array}{lllll}6 & -2.321282 & -1.636890 & 1.866148\end{array}$

$\begin{array}{lllll}6 & -1.201265 & -1.646591 & 0.944631\end{array}$

$6 \quad 0.022072 \quad-2.849928 \quad-0.681162$

$\begin{array}{lllll}6 & 0.434467 & -4.148667 & -1.084998\end{array}$

$1 \quad 0.234785 \quad-4.958603-0.391523$

$\begin{array}{lllll}6 & 1.035287 & -4.353178 & -2.302594\end{array}$

$\begin{array}{llll}1 & 1.344102 & -5.351492 & -2.601299\end{array}$

$\begin{array}{lllll}6 & 1.214468 & -3.266968 & -3.173670\end{array}$

$1 \quad 1.628020 \quad-3.394753 \quad-4.168702$

$\begin{array}{lllll}6 & 0.823802 & -2.010380 & -2.749215\end{array}$

$\begin{array}{lllll}6 & 1.846368 & 2.890022 & -1.724515\end{array}$

$\begin{array}{lllll}1 & 2.681236 & 2.415767 & -1.193799\end{array}$

$\begin{array}{llll}1 & 2.240531 & 3.771920 & -2.236453\end{array}$

$\begin{array}{llll}1 & 1.494014 & 2.178250 & -2.483037\end{array}$

$\begin{array}{lllll}6 & 0.875096 & -0.816560 & -3.650894\end{array}$

$\begin{array}{llll}1.227203 & 0.092610 & -3.134867\end{array}$

$1 \quad-0.128514-0.578381-4.019482$

$1 \quad 1.548006 \quad-0.977442 \quad-4.498116$

$\begin{array}{lllll}6 & -2.661940 & 1.082638 & -2.017251\end{array}$

$1 \quad-2.6513391 .543854 \quad-0.579703$

$\begin{array}{llll}8 & -1.662684 & 0.228296 & -2.120899\end{array}$

$\begin{array}{llll}6 & -2.535317 & 2.403042 & -2.744282\end{array}$

$\begin{array}{llll}1 & -1.487857 & 2.714827 & -2.756992\end{array}$

$\begin{array}{lllll}1 & -2.865337 & 2.306732 & -3.788269\end{array}$

$1 \quad-3.126510 \quad 3.205052 \quad-2.288841$

$\begin{array}{llll}6 & -3.993133 & 0.510759 & -1.766996\end{array}$

$\begin{array}{lllll}6 & -5.181044 & 1.255011 & -1.906951\end{array}$

$\begin{array}{llll}6 & -4.076660 & -0.782519 & -1.216419\end{array}$

$\begin{array}{lllll}6 & -6.397550 & 0.726609 & -1.502093\end{array}$

$\begin{array}{llll}1 & -5.146365 & 2.254237 & -2.334984\end{array}$

$6 \quad-5.297257-1.303174 \quad-0.808626$

$1 \quad-3.158632-1.356528-1.115767$

$6 \quad-6.462361 \quad-0.550467-0.939738$

$1 \quad-7.305071 \quad 1.313808-1.620880$

$1 \quad-5.336034-2.296473 \quad-0.367967$

$1 \quad-7.415953 \quad-0.953307 \quad-0.609596$

$\begin{array}{llll}6 & 2.271296 & 1.490094 & 1.467718\end{array}$

$\begin{array}{llll}6 & 3.425320 & 2.282386 & 1.497044\end{array}$

$\begin{array}{lllll}6 & 1.180908 & 1.872011 & 2.264046\end{array}$

$\begin{array}{llll}6 & 3.491090 & 3.411373 & 2.311439\end{array}$

$\begin{array}{llll}1 & 4.279136 & 2.024508 & 0.875879\end{array}$

$\begin{array}{llll}6 & 1.254027 & 2.987501 & 3.089348\end{array}$

$\begin{array}{llll}1 & 0.253585 & 1.303275 & 2.208898\end{array}$

$\begin{array}{llll}6 & 2.410588 & 3.763594 & 3.113549\end{array}$

$14.394406 \quad 4.015569 \quad 2.317085$

$\begin{array}{llll}1 & 0.394557 & 3.262963 & 3.694712\end{array}$

$1 \quad 2.464576 \quad 4.643910 \quad 3.748146$

$6 \quad \begin{array}{llll}3.718603 & -0.117757 & -0.433021\end{array}$

$\begin{array}{llll}6 & 4.841167 & -0.439128 & 0.345395\end{array}$

$\begin{array}{llll}6 & 3.888498 & 0.044043 & -1.808527\end{array}$

$6 \quad 6.092233 \quad-0.587787 \quad-0.242120$

$14.724690 \quad-0.581604 \quad 1.417809$

$\begin{array}{lllll}6 & 5.139734 & -0.109492 & -2.402729\end{array}$

$1 \quad 3.026428 \quad 0.290728 \quad-2.421083$

$\begin{array}{lllll}6 & 6.243788 & -0.426091 & -1.619203\end{array}$

$\begin{array}{llll}1 & 6.951231 & -0.837601 & 0.374417\end{array}$

$\begin{array}{llll}1 & 5.247260 & 0.013377 & -3.476915\end{array}$

$1 \quad 7.220882 \quad-0.550821 \quad-2.077499$ 


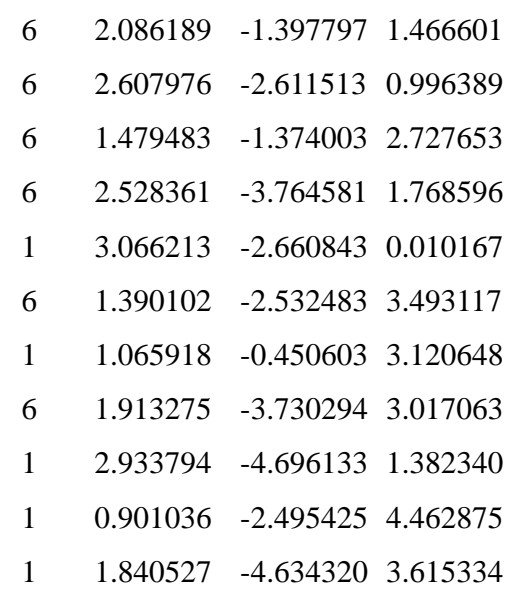

A-4 ${ }^{-2}$

$44 \quad-0.473202-0.284616 \quad-0.372283$

$7 \quad-0.507426 \quad 1.706033 \quad-1.162701$

$\begin{array}{llll}7 & -2.474507 & 2.295631 & 0.057469\end{array}$

$\begin{array}{lllll}7 & -2.493335 & -0.049630 & -0.266729\end{array}$

$\begin{array}{lllll}7 & -3.236787 & -2.272448 & -0.704528\end{array}$

$7 \quad-0.836406-2.386113-0.345124$

$\begin{array}{llll}6 & 0.401495 & 2.119509 & -2.107329\end{array}$

$\begin{array}{llll}6 & 0.558511 & 3.451155 & -2.448796\end{array}$

$\begin{array}{llll}1 & 1.304426 & 3.712584 & -3.192679\end{array}$

$\begin{array}{lllll}6 & -0.230555 & 4.429891 & -1.844058\end{array}$

$1 \quad-0.098694 \quad 5.481671 \quad-2.077030$

$\begin{array}{llll}6 & -1.228654 & 4.016799 & -0.989176\end{array}$

$\begin{array}{lllll}1 & -1.930593 & 4.723700 & -0.555371\end{array}$

$\begin{array}{lllll}6 & -1.375386 & 2.652280 & -0.707794\end{array}$

$\begin{array}{llll}6 & -3.104821 & 1.080020 & 0.084100\end{array}$

$\begin{array}{lllll}6 & -4.484322 & 0.859019 & 0.430918\end{array}$

$\begin{array}{llll}6 & -5.524172 & 1.680437 & 0.866695\end{array}$

$\begin{array}{llll}1 & -5.377454 & 2.741407 & 1.060644\end{array}$

$\begin{array}{llll}6 & -6.777500 & 1.102256 & 1.063291\end{array}$

$\begin{array}{llll}1 & -7.600563 & 1.719427 & 1.412589\end{array}$

$\begin{array}{llll}6 & -6.994646 & -0.258622 & 0.817830\end{array}$

$\begin{array}{llll}1 & -7.984693 & -0.676249 & 0.976123\end{array}$

$\begin{array}{lllll}6 & -5.959329 & -1.082271 & 0.372982\end{array}$

$\begin{array}{lllll}1 & -6.118985 & -2.137893 & 0.172987\end{array}$

$\begin{array}{lllll}6 & -4.705680 & -0.518034 & 0.186171\end{array}$

$6 \quad-3.438666 \quad-1.064648 \quad-0.268551$

$\begin{array}{lllll}6 & -2.020577 & -2.825707 & -0.932841\end{array}$

$\begin{array}{lllll}6 & -2.048897 & -3.993197 & -1.736088\end{array}$
$1 \quad-2.986744 \quad-4.220226-2.231543$

$\begin{array}{lllll}6 & -0.946900 & -4.801674 & -1.829776\end{array}$

$1 \quad-0.946411 \quad-5.687901-2.457056$

$6 \quad 0.127411 \quad-4.515959-0.973770$

$1 \quad 0.943728 \quad-5.222045 \quad-0.848546$

$\begin{array}{lllll}6 & 0.131309 & -3.364623 & -0.209951\end{array}$

$\begin{array}{llll}6 & 1.200088 & 1.069410 & -2.799955\end{array}$

$1 \quad 1.441936 \quad 1.386886 \quad-3.818853$

$1 \quad 0.644683 \quad 0.124153 \quad-2.818860$

$1 \quad 2.149757 \quad 0.868161 \quad-2.293080$

$\begin{array}{llll}6 & 1.144216 & -3.223278 & 0.875529\end{array}$

$1 \quad 1.426643 \quad-4.216799 \quad 1.235955$

$1 \quad 0.726656 \quad-2.658670 \quad 1.710467$

$\begin{array}{llll}1 & 2.069728 & -2.730859 & 0.569746\end{array}$

$\begin{array}{lllll}15 & 1.698708 & -0.037104 & 0.317626\end{array}$

$\begin{array}{llll}6 & 2.221028 & 1.753232 & 0.388137\end{array}$

$\begin{array}{lllll}6 & 3.264890 & 2.315405 & -0.356156\end{array}$

$\begin{array}{llll}6 & 1.451623 & 2.613445 & 1.185422\end{array}$

$\begin{array}{llll}6 & 3.545109 & 3.679315 & -0.287143\end{array}$

$1 \quad 3.869131 \quad 1.690049 \quad-1.007483$

$\begin{array}{llll}6 & 1.728085 & 3.973446 & 1.256208\end{array}$

$\begin{array}{llll}1 & 0.614944 & 2.204778 & 1.751084\end{array}$

$\begin{array}{llll}6 & 2.781362 & 4.513942 & 0.520950\end{array}$

$1 \quad 4.364459 \quad 4.087387 \quad-0.874131$

$\begin{array}{llll}1 & 1.113706 & 4.614948 & 1.883250\end{array}$

$\begin{array}{llll}1 & 3.000606 & 5.577085 & 0.575196\end{array}$

$\begin{array}{llll}6 & 1.915052 & -0.442140 & 2.121212\end{array}$

$\begin{array}{lllll}6 & 3.056684 & -1.007039 & 2.697534\end{array}$

$\begin{array}{lllll}6 & 0.804441 & -0.205603 & 2.944971\end{array}$

$\begin{array}{llll}6 & 3.092601 & -1.311782 & 4.056660\end{array}$

$\begin{array}{lllll}1 & 3.918197 & -1.249070 & 2.082865\end{array}$

$\begin{array}{lllll}6 & 0.845309 & -0.494709 & 4.304981\end{array}$

$\begin{array}{llll}1 & -0.111225 & 0.169547 & 2.483441\end{array}$

$\begin{array}{lllll}6 & 1.992525 & -1.049109 & 4.867199\end{array}$

$1 \quad 3.986265 \quad-1.7630914 .480722$

$1 \quad-0.028442 \quad-0.302948 \quad 4.922610$

$\begin{array}{lllll}1 & 2.023574 & -1.288884 & 5.926664\end{array}$

$\begin{array}{lllll}6 & 3.191850 & -0.795261 & -0.492059\end{array}$

$\begin{array}{lllll}6 & 2.942140 & -1.673812 & -1.554749\end{array}$

$6 \quad 4.527060-0.533936-0.150499$

$\begin{array}{lllll}6 & 3.984764 & -2.289810 & -2.240747\end{array}$

$1 \quad 1.903190 \quad-1.867872-1.829422$

$\begin{array}{llll}6 & 5.573361 & -1.145942 & -0.835974\end{array}$ 


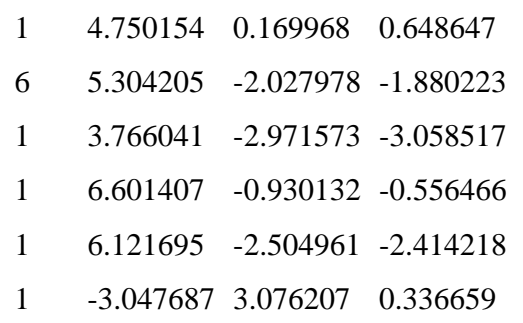

\section{A-TS3 $\mathbf{I}$-PS}

$\begin{array}{lllll}44 & 0.586114 & -0.038307 & -0.723672\end{array}$

$\begin{array}{lllll}15 & 2.883026 & 0.307203 & -0.149526\end{array}$

$\begin{array}{lllll}7 & 0.160064 & 2.015040 & -0.622143\end{array}$

$\begin{array}{llll}7 & -0.879036 & 1.899826 & 1.572722\end{array}$

$\begin{array}{lllll}7 & 0.168717 & -0.242098 & 1.196926\end{array}$

$\begin{array}{lllll}7 & 0.699404 & -2.588735 & 1.509763\end{array}$

$\begin{array}{lllll}7 & 0.929426 & -2.129323 & -0.863935\end{array}$

$\begin{array}{lllll}6 & 0.474962 & 2.808037 & -1.688990\end{array}$

$\begin{array}{llll}6 & 0.140541 & 4.150636 & -1.733765\end{array}$

$\begin{array}{lllll}1 & 0.430861 & 4.734496 & -2.600754\end{array}$

$\begin{array}{llll}6 & -0.590622 & 4.715925 & -0.688119\end{array}$

$1 \quad-0.885209 \quad 5.760416 \quad-0.715114$

$\begin{array}{llll}6 & -0.930715 & 3.917492 & 0.378801\end{array}$

$\begin{array}{llll}1 & -1.494925 & 4.291824 & 1.225893\end{array}$

$\begin{array}{llll}6 & -0.519218 & 2.572634 & 0.417518\end{array}$

$\begin{array}{llll}6 & -0.481075 & 0.673138 & 1.940390\end{array}$

$\begin{array}{llll}6 & -0.774227 & 0.114210 & 3.252156\end{array}$

$\begin{array}{lllll}6 & -1.358270 & 0.652441 & 4.392371\end{array}$

$\begin{array}{llll}1 & -1.724711 & 1.675462 & 4.403499\end{array}$

$\begin{array}{llll}6 & -1.449056 & -0.164998 & 5.521466\end{array}$

$\begin{array}{lllll}1 & -1.903242 & 0.224884 & 6.428146\end{array}$

$\begin{array}{llll}6 & -0.961248 & -1.474764 & 5.508239\end{array}$

$1 \quad-1.042554 \quad-2.0821396 .405185$

$\begin{array}{lllll}6 & -0.366494 & -2.010126 & 4.362464\end{array}$

$\begin{array}{lllll}1 & 0.025524 & -3.023071 & 4.340109\end{array}$

$\begin{array}{lllll}6 & -0.277904 & -1.198614 & 3.243883\end{array}$

$\begin{array}{lllll}6 & 0.272293 & -1.433078 & 1.909386\end{array}$

$\begin{array}{lllll}6 & 1.048849 & -2.939897 & 0.237197\end{array}$

$\begin{array}{lllll}6 & 1.510957 & -4.266424 & 0.107875\end{array}$

$\begin{array}{lllll}1 & 1.607810 & -4.840603 & 1.022539\end{array}$

$\begin{array}{lllll}6 & 1.797817 & -4.787706 & -1.132037\end{array}$

$1 \quad 2.162713 \quad-5.804824 \quad-1.240207$

$\begin{array}{llll}6 & 1.562550 & -3.989458 & -2.252122\end{array}$
$1 \quad 1.708269-4.374694 \quad-3.256499$

$\begin{array}{lllll}6 & 1.103132 & -2.688889 & -2.097206\end{array}$

$\begin{array}{lllll}6 & 1.162375 & 2.158382 & -2.840385\end{array}$

$\begin{array}{lllll}1 & 2.157612 & 1.792658 & -2.558777\end{array}$

$\begin{array}{lllll}1 & 1.275888 & 2.858795 & -3.671651\end{array}$

$1 \quad 0.576454 \quad 1.300036 \quad-3.195186$

$\begin{array}{lllll}6 & 0.732269 & -1.895747 & -3.308103\end{array}$

$1 \quad 0.973808 \quad-0.833231 \quad-3.213879$

$1 \quad-0.352057-1.924314 \quad-3.454304$

$1 \quad 1.231244 \quad-2.293467 \quad-4.196393$

$\begin{array}{lllll}6 & -2.425164 & 0.157833 & -1.549282\end{array}$

$1 \quad-2.772192 \quad 0.774903 \quad-0.420658$

$8 \quad-1.112534-0.349284-1.595562$

$6 \quad-2.620558 \quad 1.236102 \quad-2.603771$

$1 \quad-1.919422 \quad 2.060821 \quad-2.438463$

$1 \quad-2.4651890 .857223 \quad-3.625695$

$1 \quad-3.629927 \quad 1.660565 \quad-2.549369$

$6 \quad-3.362324-1.011578-1.679651$

$6 \quad-4.540619 \quad-0.990395 \quad-2.438202$

$6 \quad-3.072750-2.188057-0.966455$

$\begin{array}{lllll}6 & -5.395419 & -2.089882 & -2.472485\end{array}$

$1-4.807096 \quad-0.099568 \quad-3.001453$

$6 \quad-3.924805-3.283822-0.996754$

$1 \quad-2.155531-2.220816-0.381490$

$6 \quad-5.096469-3.242955-1.753759$

$1 \quad-6.307867 \quad-2.037460 \quad-3.063011$

$1 \quad-3.673901-4.177590-0.429122$

$1 \quad-5.766062-4.099005-1.780286$

$\begin{array}{llll}6 & 3.287523 & 2.069937 & 0.176092\end{array}$

$\begin{array}{lllll}6 & 4.203107 & 2.789169 & -0.602517\end{array}$

$\begin{array}{llll}6 & 2.539292 & 2.770163 & 1.134744\end{array}$

$\begin{array}{lllll}6 & 4.371096 & 4.159767 & -0.417494\end{array}$

$\begin{array}{lllll}1 & 4.785630 & 2.280249 & -1.366299\end{array}$

$\begin{array}{llll}6 & 2.717980 & 4.134507 & 1.329279\end{array}$

$\begin{array}{llll}1 & 1.784629 & 2.245226 & 1.716606\end{array}$

$\begin{array}{lllll}6 & 3.635712 & 4.835869 & 0.550577\end{array}$

$1 \quad 5.085048 \quad 4.698928 \quad-1.034655$

$\begin{array}{llll}1 & 2.123038 & 4.651408 & 2.077768\end{array}$

$\begin{array}{llll}1 & 3.768847 & 5.904762 & 0.691868\end{array}$

$6 \quad 4.201206 \quad-0.176642-1.337710$

$\begin{array}{lllll}6 & 5.543884 & -0.280280 & -0.945864\end{array}$

$\begin{array}{lllll}6 & 3.875722 & -0.415521 & -2.673769\end{array}$

$\begin{array}{llll}6 & 6.525391 & -0.620642 & -1.869685\end{array}$ 


\begin{tabular}{|c|c|c|c|}
\hline & 5.815262 & -0.093455 & 0.091050 \\
\hline & 4.856162 & -0.758982 & -3.602066 \\
\hline & 2.839071 & -0.329180 & -2.985623 \\
\hline & 6.183108 & -0.863424 & -3.200152 \\
\hline & 7.561840 & -0.696252 & -1.552081 \\
\hline & 4.579683 & -0.946290 & -4.636336 \\
\hline & 6.952009 & -1.132750 & -3.919207 \\
\hline & 3.433964 & -0.607680 & 1.347666 \\
\hline & 3.838822 & -1.942537 & 1.197344 \\
\hline & 3.284216 & -0.101063 & 2.644652 \\
\hline & 4.079767 & -2.743696 & 2.307623 \\
\hline & 3.954225 & -2.362372 & 0.199270 \\
\hline & 3.523753 & -0.905761 & 3.754844 \\
\hline & 2.987518 & 0.932109 & 2.798798 \\
\hline & 3.915749 & -2.231280 & 3.591493 \\
\hline & 4.386747 & -3.776916 & 2.165073 \\
\hline & 3.398506 & -0.491513 & 4.751807 \\
\hline & 4.095895 & -2.859736 & 4.459191 \\
\hline & -7.839062 & 0.534318 & -1.252623 \\
\hline & -6.969332 & 1.618406 & -1.342504 \\
\hline & -5.829636 & 1.669372 & -0.545202 \\
\hline & -5.541437 & 0.634796 & 0.347073 \\
\hline & -6.418561 & -0.447092 & 0.432623 \\
\hline & -7.560161 & -0.498397 & -0.359909 \\
\hline & -8.725060 & 0.489166 & -1.880783 \\
\hline & -7.176714 & 2.425563 & -2.041498 \\
\hline & -5.131827 & 2.499362 & -0.616866 \\
\hline & -6.182994 & -1.272737 & 1.103369 \\
\hline & -8.221541 & -1.359446 & -0.298782 \\
\hline & -4.305733 & 0.699669 & 1.219989 \\
\hline & -3.934251 & -0.336961 & 1.355288 \\
\hline & -4.647150 & 1.268721 & 2.590754 \\
\hline & -3.765014 & 1.269293 & 3.241153 \\
\hline & -5.005339 & 2.298443 & 2.488970 \\
\hline$\perp$ & -5.428841 & 0.681772 & 3.084069 \\
\hline & -3.326429 & 1.489572 & 0.581911 \\
\hline & -2.254549 & 1.819387 & 1.439614 \\
\hline
\end{tabular}




\section{Reference}

(1) Sandhya, K. S.; Suresh, C. H. Organometallics 2013, 32, 2926-2933. 